This dissertation has been microfilmed exactly as received

$68-8860$

MORELAND, James Patrick, 1941-

OPTICAL HETERODYNE DETECTION OF A RANDOMLY DISTORTED SIGNAL BEAM.

The Ohio State University, Ph.D., 1967

Engineering, electrical

University Microfilms, Inc., Ann Arbor, Michigan 


\section{OPTICAL HETERODYNE DETECTION OF A \\ RANDOMLY DISTORTED SIGNAL BEAM}

\section{DISSERTATION}

Presented in Partial Fulfillment of the Requirements for the Degree Doctor of Philosophy in the Graduate

School of The Ohio State University

by

James Patrick Moreland, BSEE, M. Sc.

** * * * * * * * * * *

The Ohio State University, 1967

1967

Approved by

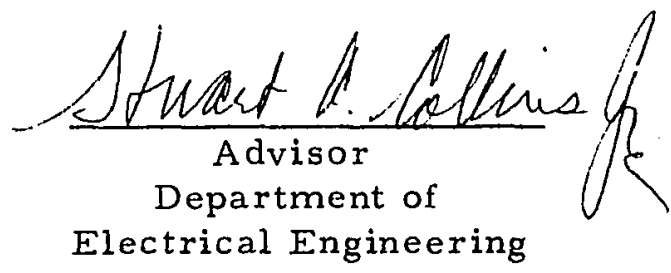




\section{ACKNOW LEDGMENTS}

The work reported in this dissertation was supported in part by Contract AF 33(615)-3419 between Air Force Avionics Laboratory, Wright-Patterson Air Force Base, Ohio and The Ohio State University Research Foundation.

The author gratefully acknowledges the help of Dr. Stuart A. Collins, Jr., his adviser and supervisor, upon whose recommendation this investigation was undertaken. Many of the ideas herein contained grew out of discussions with Dr. Collins. The suggestions of Dr. David L. Fried, of the Electro-Optical Laboratory, Autonetics Division of North American Aviation, proved quite valuable. The comments and suggestions of Dr. Curt A. Levis, Director, ElectroScience Laboratory, and Mr. Frederick W. Gebhardt of the same laboratory, helped to clarify many points. 
VITA

March 17, 1941

1964........

$1964 \ldots \ldots \ldots$

$1964 \ldots \ldots \ldots$

$1965-1967 . \ldots$

Thesis......

Reports.....
Born - Troy, New York

BSEE, The Ohio State University, Columbus, Ohio

Research Assistant, ElectroScience Laboratory, The Ohio State University, Columbus, Ohio

M. Sc. , The Ohio State University, Columbus, Ohio

Research Associate, ElectroScience Laboratory, The Ohio State University

Instructor, Department of Electrical Engineering, The Ohio State University, Columbus, Ohio

PUBLICATIONS

"A Study of Radar Absorbers to Attenuate Creeping Waves,"

"Further Studies of Echo Area Contributions of Creeping Waves," ElectroScience Laboratory Report 1815-5, $31 \mathrm{July} 1965$

"Improvement of Optical Heterodyne Systems Through Spatial Filtering," ElectroScience Laboratory Report 2156-1, 10 February 1966 


\section{FIELDS OF STUDY}

Major Field: Electrical Engineering

Studies in Solid State Devices.

Professor Ibrahim H. Adawi

Studies in Microwave Components.

Professor Wendell $\mathrm{H}$. Cornetet, Jr,

Studies in Communication Theory.

Professor C. Earl Warren 
CONTENTS

Page

ACKNOWLEDGMENTS. ......................

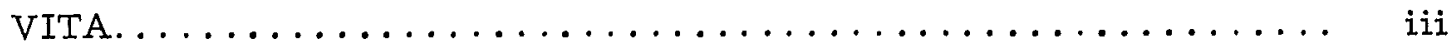

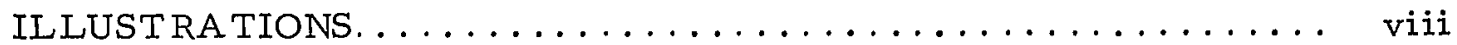

Chapter

I INTRODUCTION. .................... 1

A. Discussion of optical communication 3

B. Signal-to-noise ratio - undistorted signal 5

C. Average signal-to-noise $\quad 7$

D. Effects of random phase fluctuations 9

E. Effects of amplitude fluctuations 10

F. Time dependent detection scheme 13

G. Time independent detection schemes 15

II DERIVATION OF EQUATIONS FOR

DETERMINING OPTIMUM LOCAL

OSCILIATOR WAVE FUNCTION............ 20

A. Introduction 20

B. Derivation of equations $\quad 22$

III SOLUTION OF EQUATIONS FOR

SEVERAL SIMPLE EXAMPLES............. 32 


\section{CONTENTS (Cont.)}

Chapter

Page

IV

ALTERNA TIVE DERIVATION OF INTEGRAL ERUATION DETERMINING OPTIMUM LOCAL OSCILLATOR WAVE FUNCTION AND PHYSICAL INTERPRETATION OF RESULTS. . . . . . . . 40
A. Introduction
40
B. Karhunen-Loe've theorem
45
C. Interpretation of eigenvalues
50
D. Optical system

$\mathrm{V}$

THE OPTIMUM LOCAL OSCILLATOR

WAVE FUNCTION FOR THE CASE OF

AN ATMOSPHERICALLY DISTORTED

OPTICAL SIGNAL. ................ 57

A. Mutual coherence function $\quad 57$

B. Determination of optimum local

oscillator wave function

C. Intensity pattern of optimum local

oscillator beam and corresponding

signal-to-noise ratio

VI EQUIVALENCE OF DIFFERENT HETERODYNE DETECTION SYSTEMS AND VARIANCE OF BEAT SIGNAL POWER................

A. Discussion of different detection methods 76

B. Equivalence of different methods $\quad 79$

C. Variance of beat signal power 85

VII COMMENTS AND RECOMMENDATIONS......... 91

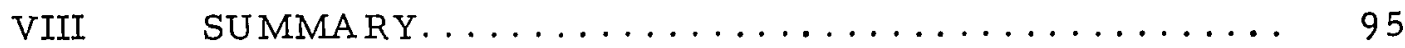




\section{CONTENTS (Cont.)}

Appendix

Page

I

MAXIMUM (S/N) -SCHWARZ INEQUALITY....... 98

II

REAIIZATION OF OPTIMUM LOCAL OSCILLATOR WAVE FUNCTION.

III

MUTUAL COHERENCE FUNCTION............ 103

IV

UNITARY TRANSFORMATIONS.

116

$\mathrm{V}$

NORMALIZATION OF S/N. .

119

REFERENCES.

121 


\section{ILLUSTRATIONS}

Figure

Page

Heterodyne detection scheme.

20

2 Received signal (for the example used

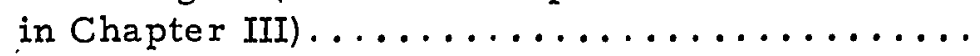

$3 \quad$ Illustration of optical system described in Chapter IV.................... 53

$4 \quad$ Receiver aperture................... 61

5(a) Normalized signal-to-noise ratio (square aperture).................. 65

5(b) Fried's normalized signal-to-noise (round aperture)................... 66

6 Local oscillator intensity patterns

(square aperture)................ 67

$7 \quad$ Percent increase in $(S / N)$ - square aperture...... 68

8 Normalized signal-to-noise ratio

( round aperture)................. 73

9 Normalized intensity patterns (round aperture).... 74

10 Time invariant detection arrangement......... 77

11 Realization of optimum local oscillator beam..... 101

12 Measurement of mutual coherence function. ...... 102

13 Dependence of $r_{0}$ upon path length $R$, and strength of turbulence as measured by $\mathrm{C}_{\mathrm{N}}^{2}$ (from Reference 1$) \ldots \ldots \ldots \ldots \ldots \ldots \ldots \ldots$ 


\section{CHAPTER I}

INTRODUCTION

This investigation is concerned with the heterodyne detection of a randomly distorted optical signal beam. The random distortion; i. e. , random fluctuations in the phase and amplitude of the received signal, may, for example, be a result of the beam having traversed a turbulent atmosphere exhibiting a randomly fluctuating index of refraction. For the case of an atmospherically distorted plane wave this problem has been considered[ 1,2$]$, but only with a non-optimal plane wave local oscillator beam. The specific problem under consideration in this investigation is to determine that time invariant optical heterodyne detection scheme which is optimal, in the sense that it maximizes the average heterodyne signal-to-noise ratio.

Optical heterodyne detection is possible because the detectors in the optical region[ 3] are "square law" detectors (i. e., the detector output current is proportional to the intensity or square of the electric field. In this process[3] the incident beam is combined on the detector's surface with a single frequency, high intensity beam called the local oscillator wave. The detector current will then contain components at frequencies equal to the difference of frequencies of the 
signal and local oscillator. These difference frequencies can, for example, be in the microwave range or lower, where efficient broadband amplification is easily obtained[ 3] .

Perhaps the most serious difficulty with direct heterodyne detection of the incident optical signal lies in the fact that the detector surface may be thousands of wavelengths wide. This then, as will be described in detail later, imposes a very severe alignment tolerance between the signal and local oscillator waves if one is to maintain a constant-signal-local oscillator phase difference. Fluctuations about this precise alignment requirement result in fading of detected signal level.

Realize now that the atmosphere, with its ever changing piessure and temperature (and hence, index of refraction), will cause distortions in both the amplitude and phase of an optical signal and then one can reasonably ask - is there any chance that direct heterodyne detection will be practical for atmospheric optical links?

This investigation partially answers this question in that it determines the maximum average signal-to-noise ratio obtainable with a time invariant detection scheme. It also prescribes the local oscillator shape required to achieve the maximum signal-to-noise ratio with 
no optical preprocessing of the received signal. It is further demonstrated that no optical processing scheme can increase the signal-tonoise ratio above this maximum value. Some related topics are also briefly considered.

To introduce the subject in more detail, the remaining sections of the introduction contain a detailed review of the problems and concepts associated with optical heterodyne detection; and present further references to the literature. First a general introduction to optical communications is presented followed by a discussion of signal-tonoise ratio and its use as the figure of merit for the evaluation of optical communication systems. The next two sections describe in qualitative, but detailed, fashion how phase and amplitude fluctuations effect the heterodyne signal. The last two sections contain a discussion of a different approach to optical heterodyne detection, namely, one using time dependent detection schemes. These are discussed and compared with time independent schemes employed in this investigation.

\section{A. Discussion of Optical \\ Communication}

There has recently been a resurgence in optical communications. This resurgence owes its existence to the fact that optical communication systems today possess the necessary prerequisites of any practical communications link. These are: (1) An efficient source of energy 
(the laser) and (2) Efficient detectors (e.g., the phototube and the semiconductor photodiodes). As each of these necessary components also exist for other regions of the electromagnetic spectrum (for example, at radio and microwave frequencies), one might then reasonably ask - why even consider optical communications? As one might suspect, the answer to this question must somehow be related to the essential difference between optical energy and these other forms namely, the wavelength (or frequency) of the associated electromagnetic wave. This conclusion is correct because it is for this reason that optical communication systems hold the following two important advantages over their counterparts at $r-f$ and microwave frequencies[4]:

(1) Increased directivity, and hence, greater antenna gain (with smaller antenna size). The larger antenna gain implies, of course, that less transmitte $r$ power is required to achieve a given level of received power, and

(2) The hugeness of the available optical spectrum $\left(10^{14}\right.$ to $10^{15} \mathrm{cps}$ ) and hence, the wide bandwidths available for the transmission of information.

The first of these advantages implies not only the possibility of efficient long distance comnunications[4], such as satellite to satellite and earth to moon, but also the ability to simultaneously operate several optical links in close proximity. With regard to the second 
advantage, it can be noted that the available bandwidth at optical frequencies is almost 20,000 times greater than that at microwave frequencies[4]. With the rapidly increasing desired rate and quantity of communications, the optical link may, then, soon be required as a replacement for the present microwave links. For example, the telephone company envisions the day when a single coast to coast laser link will simultaneously transmit thousands of "picture-telephone" conversations. Potential applications for lasers also exist in the area of computers and as extremely accurate position and velocity measuring instruments.

\section{B. Signa1-to-Noise Ratio- Undistorted Signal}

An important quantity for evaluating the performance of a com munications channel, in the absence of random fluctuations in the received signal, is the signal-to-noise ratio. In the case of heterodyne detection, it is the ratio of beat signal power to noise power. The noise, i. e., a randomly fluctuating current added to the beat signal current, may be the result of [4] external (or background) radiation falling on the detector surface; or of the quantum nature of the light flux (important for "very small" received signal powers), and of the detector itself. In the case of a photodetector[4], the internal noise is a result of the randomness of the electron-emission from the 
photosurface (shot noise); of the dark current; i. e. , that current which flows even in the absence of any external radiation; and of thermal, or resistor, noise. In this investigation it will be as sumed that the local oscillator power is of sufficient magnitude so that the detector is operating in the shot noise limited condition. In this case, the noise power, i. e., the rms value of the shot noise, is directly proportional to the local oscillator power[5]. As the beat signal power.is, ideally, directly proportional to the product of local oscillator and received signal powers[5], it follows that the signal-to-noise ratio is, for this case, directly proportional to the received signal power.

The importance of the signal-to-noise ratio is that it is this quantity which, in the case of a discrete ox digital communications system, is needed to determine the error performance of the system[6]. That is, it determines the probability that the detector makes an error in deciding what message was sent to it. In the case of waveform communication, the signal-tomoise ratio is needed to determine the mean square error between the transmitted and received wave forms[ 7$]$ - the mean square error being a measure of the "fidelity" of the received signal. 


\section{Average Signal-to-Noise}

In the presence of random fluctuations in the received signal, the signal-to-noise ratio is, by definition, no longer a deterministic function. That is, the random fluctuations in phase and amplitude of the received signal induce random fluctuations in the beat signal and hence, in the signal-to-noise ratio. A quantity which can be determined, and to which much attention has been given, is the average signal-to-noise ratio. Thus, in the case of an atmospherically distorted plane wave optical signal, Fried[1] and Gardner[2] have shown that because of the random distortion there is a limit to the achievable average signalto-noise ratio, no matter how large one makes the detector collection aperture. (Recall that, ideally, the signal-to-noise ratio is directly proportional to the collected optical signal power and hence, should increase as the size of the collection aperture is increased.) It will be important to remember in what follows that each of these analyses assumed a plane wave local oscillator beam.

Although the average signal-to-noise ratio does not provide sufficient information to fully evaluate the performance of a communications link, for reasons to be given below, it does, nevertheless, afford an easily obtainable parameter upon which some general predictions of performance can be based. For example, it sets the lower bound on the minimum obtainable probability of error or minimum 
mean square error[8]. In the work that follows, the primary interest in the average signal-to-noise ratio is in that it does provide a parameter for easily comparing the performance capabilities of different heterodyne detection schemes. In particular, the interest here is in determining that time invarient heterodyne detection scheme which yields the largest average signal-to-noise ratio.

It is worth noting here that the average signal-to-noise ratio does not determine the "average performance" of the communications channel. That is, this quantity does not determine the average probability of error, in the case of digital communications, nor the average mean square error in the case of waveform communication. The average signal-to-noise ratio would determine these quantities if they were linearly related to the signal-to-noise ratio. Unfortunately, they are highly nonlinear functions of the signal-to-noise ratio[6]. This is just what one might suspect. The basic idea here is that a signal-tonoise ratio of, say 20 to 1 , is "nearly" sufficient to completely surpress the effects of the noise. Increasing this ratio to 40 to 1 improves the performance very little. On the other hand, if the signal-to-noise ratio is "very small", then communication is "nearly" impossible. Thus, if the signal-to-noise ratio were to fluctuate between 40 and 0 , it is clear that the average performance is certainly worse than that obtained with a constant signal-to-noise ratio of 20 . 
Therefore, in order to precisely determine the average performance of the system, it would be necessary to know the probability distribution function of the beat signal power[8]. However, as described above, the average signal-to-noise ratio does provide an easily obtainable estimate of system performance and is, for this reason, the quantity to which attention is here given.

\section{Effects of Random Phase Fluctuations}

Qualitatively, the effects of random phase fluctuations (amplitude fluctuations will be discussed separately) on the signal-to-noise ratio may be understood by recalling the following: If two monochromatic beams of light with different frequencies are combined on the surface of a square law detector (e. g., a phototube) then the output current at the difference frequency, i. e., the beat signal, will have a maximum amplitude when the two wavefronts are parallel. This is because this output current is given by the sum of the beat frequency currents generated at each "point" on the detector surface, and the phases of the individual currents are given by the difference in the phases of the local oscillator and received beams at the point in question. Thus, the individual beat signals will all add in phase only when the local oscillator and received signal wavefronts are parallel. Therefore, any fluctuations in the difference in phase between two points on the signal wavefront will 
result in an average beat signal amplitude and hence, signal-to-noise ratio which is less than that in the absence of the fluctuations. Perhaps even more important, it is apparent that in the course of time the magnitude of the beat signal can fluctuate, i. e. , signal fading will appear. It is important to note that these fluctuations are always such as to reduce the magnitude of the beat signal from its maximum value. It is also apparent that as the magnitude of these phase fluctuations increase, such as might be expected when the size of the receiver aperture is increased, that the reduction in average signal-to-noise ratio from its maximum value will increase. A more complete discussion of this point is provided in Chapter III.

\section{E. Effects of Amplitude Fluctuations}

Random fluctuations in the beat signal and the associated reduction in average signal-to-noise ratio can also be caused by random amplitude fluctuations alone in the received signal. This is clear when the received optical signal power fluctuates with time. However, this can also be true even when the received optical signal power does not fluctuate with time. That is, a simple, time dependent, redistribution of the received power over the collection aperture can cause the beat signal to fluctuate with time. To see this, recall that the beat signal amplitude generated at an individual "point" on the detector surface is directly 
proportional to the product of the amplitude; $A_{i}$, of the received beam and the amplitude; $B_{i}$, of the local oscillator beam at the point (i) in question. Thus, for the case of parallel wavefronts, the net beat signal amplitude is proportional to the sum

$$
I_{0}=\sum_{i} A_{i} B_{i}
$$

over all "points" on the detector surface. Defining abstract signal and local oscillator "vectors" $\vec{A}=\left(A_{1}, A_{2}, \ldots, A_{N}\right)$ and $\vec{B}=\left(B_{1}, B_{2}, \ldots\right.$, $\left.B_{N}\right)$, respectively it follows that $[20]$

$$
I_{0}=\sum_{i} A_{i} B_{i}=\vec{A} \cdot \vec{B}
$$

Recall now that the dot product of two vectors is less than or at most equal to the product of the magnitudes of the individual vectors. Thus, $I_{0}=\vec{A} \cdot \vec{B} \leq|\vec{A}||\vec{B}|$. As is well known, the equality sign holds only when the two vectors are linearly dependent; i. e., when $B_{i}=C A_{i}$, where $C$ is an arbitrary constant.

One can now make two very important statements. First, the ratio of beat signal power (proportional to $I_{0}^{2}$ ) to noise power ( $p r o p o r-$ tional to local oscillator power, or to $|\vec{B}|^{2}$ ) is a maximum when the local oscillator and received signal beams have, not only parallel wavefronts, but also identical amplitude shapes over the detector surface. An interesting way of stating this can be obtained by employing complex 
notation to describe the amplitude and phase of the signal and local oscillator beams, i. .., $A_{1} e^{j \phi_{i}}$ and $B_{1} e^{j \theta_{i}}$, respectively. Thus, write these

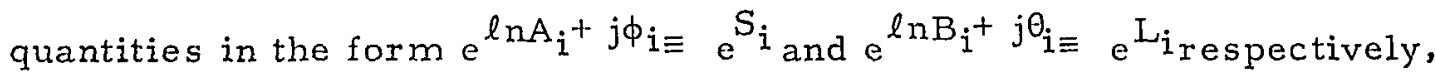
and call the quantities $S_{i}=\ln A_{i}+j \phi_{i}$ and $L_{i}=\ln B_{i}+j \theta_{i}$, the "complex phases" of the signal and local oscillator beams respectively. Then the condition for maximum signal-to-noise ratio is that the complex wavefronts of the signal and local oscillator beams be "parallel" over the detector surface. That is, the difference in complex phase at any point must be a constant, independent of the location of the point on the detector surface.

The second point to notice is that the magnitude of the beat signal for the case of parallel wavefronts, i. e., $I_{O}=\vec{A} \cdot \vec{B}$, can fluctuate with time by virtue of either the magnitude of $\vec{A}$ (which is proportional to received signal power) changing with time; or by $A_{i}$ (i. e. , the signal amplitude at "point" i) changing with time even when the signal power, $|\overrightarrow{\mathrm{A}}|^{2}$, is independent of time.

It is interesting to consider the beat signal variance. Note that it is possible for the effects of amplitude fluctuations alone, on the variance of the beat signal, to be nearly as severe as those of phase fluctuations alone. Thus, for the case of phase fluctuations, it is easy to see that $0 \leq\left|I_{0}\right| \leq|\vec{A}||\vec{B}|$. On the other hand, for the case of 
amplitude fluctuations alone, it follows that $|\overrightarrow{\mathrm{A}}| \mathrm{B}_{i} \leq\left|I_{0}\right| \leq|\overrightarrow{\mathrm{A}}||\overrightarrow{\mathrm{B}}|$. The smallest value, $|\vec{A}| B_{i}$, occurs when the entire received optical signal is concentrated about the "point" i. Thus, for very large apertures it is eveident that the possible amplitude induced variations of $\mathrm{I}_{\mathrm{O}}$ can be almost as large as the possible phase induced variations. This is true even when the received optical signal power, i. e. , $|\overrightarrow{\mathrm{A}}|^{2}$, does

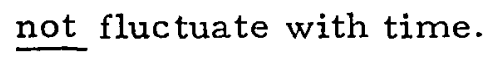

\section{F. Time Dependent Detection Schemes}

Chase[9] has examined one method for partially overcoming the effects of the random phase fluctuations of the received optical signal. Rather than using a "fixed" local oscillator beam, his scheme would employ a local oscillator beam which would track any tilting of the signal wavefront. In the event that the distortion consists of only a random tilting then this scheme will obviously yield the maximum possible signal-to-noise ratio consistent with the magnitude of the received optical signal power. If, however, there is some additional phase distortion and /or amplitude fluctuations then this scheme offers only limited improvement. Fried[ 10] suggests that such a scheme can, for "short" propagation paths where the significant part of the distortion consists of a random tilting of the signal wavefront[11], yield a shot noise limited signal-to-noise ratio improvement over the 
non-tracking system of perhaps $6 \mathrm{~dB}$. For "long" propagation paths, Fried[10] indicates that the improvement is negligible. It is interesting to note here that Fried[ 11] shows that even for "long" propagation paths, the "significant" portion of the signal distortion may be described as a random tilting, in this case not of the signal wavefront, but rather of the "complex wavefront". Recall that it is just this "conplex wavefront" which must be "matched" by the local oscillator beam in order to obtain the maximum possible signal-to-noise ratio. Thus, if it were possible to devise a means to track any tilting of the complex wavefront (a scheme which would require adjusting both the phase and amplitude of the local oscillator beam) then a significant increase in the average signal-to-noise might be obtained, not only for short propagation paths, but also for long paths.

Other time dependent detection arrangements, such as an array of "small" detectors whose output currents can be adjusted in both amplitude and/or phase, can be expected to offer still further improvement. 


\section{G. Time Independent \\ Detection Schemes}

As opposed to examining these or other time varying detection schemes, this study will determine whether a significant increase in the average signal-to-noise ratio can be obtained by employing a time invariant detection arrangement.

One of the initial thoughts here was that an improvement might be obtained by heterodyning in the focal plane of a lens through which the received signal alone is allowed to pass. An example will show that, on the surface, this scheme appears to offer a method for increasing the average signal-to-noise ratio while simultaneously reducing the signal power variance. A careful analysis will, however, show that this is not true. Thus, suppose that the fluctuations of the received signal consist of only a random tilting of an otherwise undistorted plane wave. If this signal is combined with a plane wave local oscillator beam, say parallel to the average signal wavefront, then with each receiver aperture size there will be associated a particular value of average signal-to-noise ratio and signal power variance. Suppose now that the received signal alone is passed through a lens. Then, in the focal plane of this lens, the tilting of the signal wavefront manifests itself as a change in the location of the focused spot (i. e., the Airy disk). By employing a local oscillator beam whose area is as large as the entire area over which the 
focused spot moves, it is apparent that the average beat signal power can be increased, and its variance reduced from the corresponding values obtained heterodyning with the received signal before it is passed through the lens. That is, without the use of the lens, the tilting of the signal wavefront results in non - parallel signal and local oscillator wavefronts and hence, a reduction in beat signal power. On the other hand, with the use of the lens, the tilting simply causes the focused spot to move to a new location on the detector surface where a "new" local oscillator beam is waiting for it.

At first glance this scheme might seem to offer an excellent method for "tracking" wavefront tilt. A more detailed analysis will, unfortunately, reveal that this scheme cannot yield an average signalto-noise ratio (indeed a time behavior of the beat signal itself) which cannot be obtained without the use of the lens. The basic idea here is that this scheme achieves its apparent "advantages" at the expense of an increase in local oscillator power and hence, of shot noise. To see this, recall that when the "complex wavefronts" of the signal and local oscillator are "parallel", the beat signal power is directly proportional to the product of received local oscillator and optical signal powers. Thus, without the use of the lens, let the maximum value of the beat signal power (i. e., the value obtained when the signal wavefront "lines up" with the local oscillator wavefront) be directly proportional 
to $P_{L} \cdot P_{S}$, where $P_{L}$ and $P_{S}$ are, respectively, the received local oscillator and optical signal powers. Further, suppose that, with the use of the lens, the beat signal power is to be maintained at this peak value. Assuming then that all of the received optical signal power, i. e., $P_{S}$, is contained within the focused spot, it follows that the local oscillator power contained within an area equal in size to that of the focused spot must equal $P_{L}$. But the total a rea of the local oscillator beam was, in the scheme described, to be made larger than the area of the focused optical signal spothence, inc reasing the total local oscillator power and therefore, the noise power). Thus, the average signal-to-noise ratio is, by this scheme, not increased by as much as one might have at first suspected. In fact, as will be shown below, no matter how one choses the shape of the local oscillator beam in the lens focal plane, the corresponding average signal-to-noise ratio, indeed the time behavior of the beat signal itself, can be obtained without the use of the lens. [See Reference 23 for additional discussion.] Other more complicated schemes, such as using an array of "small" lenses and heterodyning in the common focal plane of each one, could be individually analyzed. It will, however, be shown below that all optical systems which perform a time invariant, power conserving linear transformation on the received signal, yield a performance (measured in terms of average signal-to-noise ratio and time behavior of the beat signal) which is identical to that obtained 
without the use of the optical system. This important result allows one to determine the performance of all such optical systems by considering the simpler problem of heterodyning with the received signal in the absence of any optical system.

One might now argue that, at least for the case of an atmospherically distorted optical plane wave, the problem of heterodyning with the received signal itself has already received sufficient consideration, that is, by Fried[1] and Gardner[2]. Recall, however, that the previous analyses of this problem assumed a plane wave local oscillator beam. Is this, however, the optimum shape for the local oscillator beam? That is, does it yield the largest possible average signal-tonoise ratio?

In the absence of any fluctuations of the received plane wave signal, a plane wave local oscillator beam is the optimum local oscillator beam. When the fluctuations are present and are such that the average received signal is a plane wave, one is tempted to say that the optimum local oscillator beam is still a plane wave. This conclusion is, however, not necessarily correct. Indeed, the results of this report allow one to determine the shape of that fixed local oscillator beam which maximizes the average signal-to-noise ratio for a given size receiver aperture. The results appear in the form of an integral equation to be satisfied by this optimum local oscillator beam. It is shown that 
the solution of this integral equation for both the phase front and amplitude shape of the optimum local oscillator beam depend on only the mutual coherence function of the received beam.

This result is derived in Chapter II. Chapter III discusse's the solution for several simple examples, and Chapter IV offers an alternative derivation and physical interpretation of the results of Chapter II. In Chapter $\mathrm{V}$ the optimum local oscillator beam is determined explicitly for the case of an atmospherically distorted optical plane wave. Furthermore, the corresponding maximum average signal-to-hoise ratio dependence on receiver aperture size is given and is compared with that obtainable with a plane wave local oscillator wave as reported by Fried[1]. In Chapter VI it is shown that no additional improvement in the average signal-to-noise ratio can be obtained by performing any time-invariant preprocessing operation on the received signal, before combining it with a local oscillator beam. Chapter VI includes some general comments and recommendations for future work. 
CHAPTER II

DERIVATION OF EQUATIONS FOR DETERMINING

OPTIMUM LOCAL OSCILLATOR WAVE FUNCTION

\section{A. Introduction}

In this Chapter the equations for determining the optimum local oscillator wave function are derived. Note that no particular form is assumed for the statistics of the signal distortion. The numerical evaluation of the results obtained will, however, depend on the assumed statistics.

The physical situation under consideration is as follows: Suppose that a source transmits, into a turbulent atmosphere, an optical beam characterized by a time invariant amplitude and phase and angular frequency $\omega_{S}$ (see Fig. 1). As a result of index of refraction fluctuations

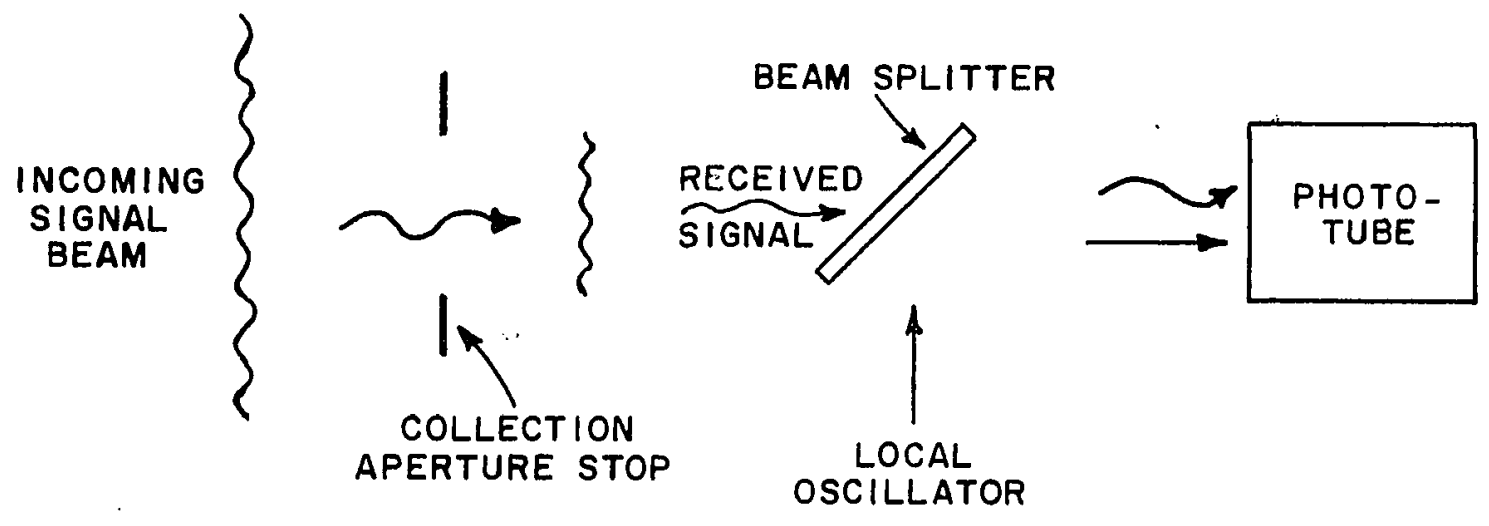

Fig. 1--Heterodyne detection scheme. 
(with time) along the propagation path, this beam appears at the receiver characterized by a time-varying amplitude and phase. This beam, or one derived from it, say by passing it through a lens, is then to be combined with a local oscillator beam, characterized by a time-invariant amplitude and phase and angular frequency $\omega_{L}$, on the surface of a square law detector. As a result of the random distortion of the received beam, the output current at the difference frequency will exhibit a time varying amplitude and phase. Our concern here is with the average power of this beat signal over a given interval of time $T$. This average power will be seen to depend not on the detailed behavior of the amplitude and phase fluctuations of the received beam, but rather on only certain averages of these fluctuations over the time interval $T$, specifically, on the mutual coherence function. It will also depend on the choice of the amplitude and phase of the local oscillator beam. The local oscillator power will be assumed to be of sufficient magnitude so that, as described previously, the noise power is directly proportional to the local oscillator power. The problem of interest is, then, when given the necessary averages associated with the received beam, how should the phase and amplitude of the local oscillator beam be shaped over the detector surface so as to maximize the average signal-to-noise ratio? 


\section{B. Derivation of Equations}

Since the noise power is directly proportional to the local oscil lator power, maximization of the average signal-to-noise ratio is equivalent to maximizing the average beat signal power subject to the condition of constant local oscillator power. The procedure, then, is to employ the calculus of variations combined with the technique of Lagrange multipliers to determine the conditions to be met by the optimum local oscillator wave function.

The heterodyne detection configuration is shown in Fig. 1. The collection aperture, which defines the illuminated portion of the photosurface, has arbitrary shape. A point on the photosurface is chosen as the origin of a coordinate system in the plane of the photodetector surface. The vector $\vec{x}$ denotes a position in this plane.

The incoming signal beam is given by the expression

$$
\begin{aligned}
E_{S} & =A(\vec{x}, t) \cos \left(\omega_{S} t+\phi(\vec{x}, t)\right) \\
& =\operatorname{Re} U(\vec{x}, t) e^{j \omega_{S} t}
\end{aligned}
$$

where the incoming wave function $U(\vec{x}, t)$, is

$$
U(\vec{x}, t)=A(\vec{x}, t) e^{j \phi(\vec{x}, t)}
$$

The time variation of $U$ is assumed to be due only to the random changes in the index of refraction, with time, along the propagation path. The received signal wave function is $U(\vec{x}, t) W(\vec{x})$, where 
$W(\vec{x})$ defines the collection aperture, i. e., $W(\vec{x})=1$ for points within the aperture and $W(\vec{x})=0$ for points outside the aperture. The local oscillator field is denoted by

$$
\begin{aligned}
E_{L} & =B(\vec{x}) \cos \left(\omega_{L} t+\theta(\vec{x})\right) \\
& =\operatorname{Re} V(\vec{x}) e^{j \omega_{L} t}
\end{aligned}
$$

where

$$
V(\vec{x})=B(\vec{x}) e^{j \theta(\vec{x})}
$$

is the local ascillator wave function. The photodetector output current at the difference frequency,

$$
\omega=\omega_{S}-\omega_{L}
$$

is

$$
I=R e e^{j \omega t} \eta \int W(\vec{x}) V^{*}(\vec{x}) U(\vec{x}, t) d \vec{x}
$$

where $\eta$ is constant, characteristic of the photodetector.

The average beat signal power over one period of the difference frequency is

$$
P=\frac{1}{2} \eta^{2} \iint W(\vec{x}) V^{*}(\vec{x}) U(\vec{x}, t) W\left(\vec{x}^{\prime}\right) V\left(\vec{x}^{\prime}\right) U^{*}\left(\overrightarrow{x^{\prime}}, t\right) d \vec{x} d \overrightarrow{x^{1}},
$$

where it has been assumed that $U$ is "nearly" constant over each period of the difference frequency. 
The average value of $P$, denoted by $\langle P\rangle$, over a time interval $T$, long compared with one period of the difference frequency, is

$$
\begin{aligned}
P_{0}=\langle P\rangle=\frac{1}{2} \eta^{2} \iint & W(\vec{x}) W\left(\vec{x}^{\prime}\right) V^{*}(\vec{x}) V\left(\overrightarrow{x^{\prime}}\right) \\
& \left\langle U(\vec{x}, t) U^{*}\left(\overrightarrow{x^{\prime}}, t\right)\right\rangle d \overrightarrow{d x} d \overrightarrow{x^{\prime}} .
\end{aligned}
$$

The photoelectron shot noise power is, for the case where the local oscillator power is much greater than the received signal power, directly proportional to the local oscillator power

$$
P_{L}=\frac{1}{2} \eta \int V(\vec{x}) V^{2 /}(\vec{x}) d \vec{x}
$$

and, in fact, the average signal noise ratio (per unit bandwidth) is given by[5]

$$
\frac{\mathrm{S}}{\mathrm{N}}=\frac{1}{2 \mathrm{e}}\left(\frac{\mathrm{P}_{\mathrm{O}}}{\mathrm{P}_{\mathrm{L}}}\right)
$$

where $e$ is the magnitude of the electronic charge.

The function $V(\vec{x})$ which maximizes $P_{0}$ subject to the condition of constant $P_{L}$ is sought. That is, by employing the calculus of variations, the condition on $V$ for which $\delta P_{0}=0$, where $\delta P_{0}$ is the variation in $P_{0}$ caused by adding a variation $\delta V$ to $V$, is required. The allowable variations in $\mathrm{V}$ are, however, not arbitrary in that $\mathrm{P}_{L}$ is to be held constant. The condition on $\delta V$ is then that $\delta P_{L}=0$, where $\delta P_{L}$ is the 
variation in $P_{L}$ caused by a variation $\delta V$ in $V$. This constraint can be included by using the method of Lagrange multipliers. It follows then that the condition on $V$ for maximum $P_{0}$ is met when

$$
\frac{\delta P_{0}}{\frac{1}{2} \eta^{2}}+(-\lambda) \frac{\delta P_{I_{L}}}{\frac{1}{2} \eta}=0,
$$

where $(-\lambda)$ is the undetermined Lagrange multiplier. Then, from Eq. (7)

$$
\begin{aligned}
P_{0}+\delta P_{0} & =\frac{1}{2} \eta^{2} \iint W(\vec{x}) W\left(\vec{x}^{\prime}\right) \\
& {[V(\vec{x})+\delta V(\vec{x})] *\left[V\left(\vec{x}^{\prime}\right)+\delta V\left(\vec{x}^{\prime}\right)\right] } \\
& <U(\vec{x}, t) U^{* *}\left(\overrightarrow{x^{\prime}}, t\right)>d \vec{x} d \vec{x}^{\prime}
\end{aligned}
$$

and hence to first order in $\delta \mathrm{V}$,

$$
\begin{aligned}
& \begin{aligned}
& \frac{\delta P_{0}}{\frac{1}{2} \eta^{2}}=\iint W(\vec{x}) W\left(\overrightarrow{x^{\prime}}\right) {\left[V^{*}(\vec{x}) \delta V\left(\vec{x}^{\prime}\right)+V\left(\overrightarrow{x^{\prime}}\right) \delta V^{* k}(\vec{x})\right] } \\
&<U(\vec{x}, t) U^{*}\left(\overrightarrow{x^{\prime}}, t\right)>d \vec{x} \overrightarrow{d x^{\prime}}
\end{aligned} \\
& =2 \operatorname{Re} \iint W(\vec{x}) W\left(\overrightarrow{x^{\prime}}\right) V\left(\overrightarrow{x^{\prime}}\right) \delta V^{*}(\vec{x}) \\
& \left\langle U(\vec{x}, t) U^{*}\left(\overrightarrow{x^{\prime}}, t\right)\right\rangle \overrightarrow{d x} d \overrightarrow{x^{\prime}},
\end{aligned}
$$

since by symmetry

$$
\begin{gathered}
\iint W(\vec{x}) W\left(\vec{x}^{\prime}\right) V^{*}(\vec{x}) \delta V\left(\vec{x}^{\prime}\right)<U(\vec{x}, t) U^{*}\left(\overrightarrow{x^{\prime}}, t\right)>d \vec{x} d \overrightarrow{x^{\prime}} \\
=\left\{\iint W(\vec{x}) W\left(\vec{x}^{\prime}\right) V\left(\vec{x}^{\prime}\right) \delta V^{* k}(\vec{x})\right. \\
\left.<U(\vec{x}, t) U^{* *}\left(\overrightarrow{x^{\prime}}, t\right)>d \vec{x} d \overrightarrow{x^{\prime}}\right\}
\end{gathered}
$$


Similarly, from Eq. (8)

$$
\begin{aligned}
\frac{\delta P_{L}}{\frac{1}{2} \eta} & =\int\left[V(\vec{x}) \delta V^{*}(\vec{x})+V^{*}(\vec{x}) \delta V(\vec{x})\right] d \overrightarrow{d x} \\
& =2 \operatorname{Re} \int V(\vec{x}) \delta V^{*}(\vec{x}) d \vec{x} .
\end{aligned}
$$

From Eqs. (12) and (13), Eq. (10) gives

$$
\begin{gathered}
2 \operatorname{Re} \iint W(\vec{x}) W\left(\overrightarrow{x^{\prime}}\right) V\left(\overrightarrow{x^{\prime}}\right) \delta V^{*}(\vec{x})<U(\vec{x}, t) U^{*}\left(\overrightarrow{x^{\prime}}, t\right)>d \overrightarrow{d x} d \overrightarrow{x^{\prime}} \\
-\lambda 2 \operatorname{Re} \int V(\vec{x}) \delta V^{*}(\vec{x}) d \vec{x}=0
\end{gathered}
$$

or

$$
\begin{aligned}
2 \operatorname{Re} \int d \vec{x} & \left\{\int W(\vec{x}) W\left(\overrightarrow{x^{\prime}}\right) V\left(\overrightarrow{x^{\prime}}\right)<U(\vec{x}, t) U^{*}\left(\overrightarrow{x^{\prime}}, t\right)>d \overrightarrow{x^{\prime}}\right. \\
& -\lambda V(\vec{x})\} \delta V^{*}(\vec{x})=0,
\end{aligned}
$$

which is satisfied, independent of the choice of $\delta \mathrm{V}(\vec{x})$, by choosing $\lambda$ so that the expression within the brackets is zero; i. e. ,

$$
\int W(\vec{x}) W\left(\vec{x}^{\prime}\right) V\left(\vec{x}^{\prime}\right)<U(\vec{x}, t) U^{*}\left(\overrightarrow{x^{\prime}}, t\right)>d \overrightarrow{x^{\prime}}-\lambda V(\vec{x})=0
$$

Stated more precisely, since $\delta V$ is a complex number, Eq. (15) demands that

$$
\int \overrightarrow{d x} \operatorname{Re}\{--\} \operatorname{Re} \delta V=0
$$

and

$$
\int \overrightarrow{d x} \operatorname{Im}\{---\} \operatorname{Im} \delta V=0
$$


which is satisfied by choosing both the real and imaginary parts of $\{--\}$ equal to zero, as given by Eq. (16).

The determination of the local oscillator wave function which maximizes the average beat signal power over the time interval $T$, subject to the condition of constant local oscillator power, has now been reduced to the solution of an integral equation, Eq. (16). This equation, related to the Fredholm Equation, is known to have solutions, called eigenfunctions, for only certain discrete values of $\lambda$, called eigenvalues. The appearance of the function $W(\vec{x})$ on the left hand side of Eq. (16) simply indicates the rather obvious fact that $V(\vec{x})$ should be zero where there is no signal beam.

It will now be shown that all the eigenvalues are real and in so doing the desired eigenfunction will become apparent. Thus, let $\lambda_{i}$ be an eigenvalue of Eq. $(16)$ and $V_{i}(\vec{x})$ the corresponding eigenfunction. Substituting this solution, multiplying by $v_{i}^{*}(\vec{x})$, and integrating the resulting equation with respect to $\vec{x}$ yields

$$
\begin{gathered}
\left.\iint W(\vec{x}) W\left(\overrightarrow{x^{\prime}}\right) V_{i}^{*}(\vec{x}) V_{i}\left(\vec{x}^{\prime}\right)<U(\vec{x}, t) U^{*}\left(\overrightarrow{x^{\prime}}, t\right)\right\rangle \overrightarrow{d x} \overrightarrow{d x^{\prime}} \\
-\lambda_{i} \int V_{i}(\vec{x}) V_{i}^{*}(\vec{x}) d \overrightarrow{d x}=0
\end{gathered}
$$


In view of Eqs. (7) and (8), Eq. (17) reads

$$
\frac{\left(P_{0}\right)_{i}}{\frac{1}{2} \eta^{2}}=\lambda_{i} \frac{\left(P_{L}\right)_{i}}{\frac{1}{2} \eta}
$$

or

(18)

$$
\lambda_{i}=\frac{1}{\eta}\left(\frac{P_{0}}{P_{I}}\right)_{i}
$$

But the right hand side of Eq. $(18)$ is real; i. e., $P_{0}=P_{0}^{*}$ and $P_{L}=P_{L}^{*}$ and hence $\lambda_{i}$ must be real. Furthermore, $\lambda_{i}$ is directly proportional to the ratio of average signal power to local oscillator power and hence to the average signal-to-noise ratio. Thus, the desired eigenfunction, $V(x)$, is that corresponding to the largest eigenvalue, $\lambda_{\max }$, of Eq. (16).

Equation (16) is really two equations in one; i. e. , both the real and imaginary paxts must separately add to zero. Equating the imaginary part to zero will yield an explicit solution for the phase front of the local oscillator wave function and equating the real part to zero will yield an integral equation to be satisfied by the corresponding amplitude shape of the local oscillator wave function.

Thus, from Eqs. (1) and (3), Eq. (16) may be written

$$
\begin{aligned}
& \int W(\vec{x}) W\left(\overrightarrow{x^{\prime}}\right) B\left(\vec{x}^{\prime}\right) e^{j\left[\theta\left(\overrightarrow{x^{\prime}}\right)-\theta(\vec{x})\right]} \\
& <A(\vec{x}, t) A\left(\vec{x}^{\prime}, t\right) e^{j\left[\phi(\vec{x}, t)-\phi\left(\vec{x}^{\prime}, t\right)\right]}>d \overrightarrow{x^{\prime}}-\lambda B(\vec{x})=0 .
\end{aligned}
$$


Equating the imaginary part of Eq. (19) to zero gives

$$
\begin{aligned}
& \int W(\vec{x}) W\left(\vec{x}^{\prime}\right) B\left(\overrightarrow{x^{\prime}}\right)\left\{\cos \left[\theta\left(\overrightarrow{x^{\prime}}\right)-\theta(\vec{x})\right]<A(\vec{x}, t) A\left(\overrightarrow{x^{\prime}}, t\right)\right. \\
& \sin \left[\phi(\vec{x}, t)-\phi\left(\overrightarrow{x^{\prime}}, t\right)\right]>+\sin \left[\theta\left(\overrightarrow{x^{\prime}}\right)-\theta(\vec{x})\right] \\
& <A(\vec{x}, t) A\left(\overrightarrow{\left.x^{\prime}, t\right)} \cos \left[\phi(\vec{x}, t)-\phi\left(\vec{x}^{\prime}, t\right)\right]>\right\} \quad \overrightarrow{d x^{\prime}}=0 .
\end{aligned}
$$

The left hand side of Eq. (20) can be made zero, for all $\vec{x}$, by choosing the expression within the brackets equal to zero; i. e., by choosing

$$
\begin{aligned}
\tan [\theta(x)- & \left.\theta\left(x^{\prime}\right)\right] \\
= & \frac{\left\langle A(\vec{x}, t) A\left(\overrightarrow{x^{\prime}}, t\right) \sin \left[\phi(\vec{x}, t)-\phi\left(\overrightarrow{x^{\prime}}, t\right)\right]\right\rangle}{\left.<A(\vec{x}, t) A\left(\overrightarrow{x^{\prime}}, t\right) \cos \left[\phi(\vec{x}, t)-\phi\left(\vec{x}^{\prime}, t\right)\right]\right\rangle}
\end{aligned}
$$

Equating the real part of Eq. (19) to zero gives

$$
\begin{aligned}
& \int W(\vec{x}) W\left(\overrightarrow{x^{\prime}}\right) B\left(\overrightarrow{x^{\prime}}\right)\left\{\cos \left[\theta(\vec{x})-\theta\left(\overrightarrow{x^{\prime}}\right)\right]<A(\vec{x}, t) A\left(\overrightarrow{x^{\prime}}, t\right)\right. \\
& \cos \left[\phi(\vec{x}, t)-\phi\left(\overrightarrow{x^{\prime}}, t\right)\right]>+\sin \left[\theta(\vec{x})-\theta\left(\overrightarrow{x^{\prime}}\right)\right] \\
& \left.<A(\vec{x}, t) A\left(\vec{x}^{\prime}, t\right) \sin \left[\phi(\vec{x}, t)-\phi\left(\overrightarrow{x^{\prime}}, t\right)\right]>\right\} d \overrightarrow{x^{\prime}}-\lambda B(x)=0 .
\end{aligned}
$$

Equations (21) and (22) may be written in more compact form by defining

$$
\begin{aligned}
& \bar{S}\left(\vec{x}, \overrightarrow{x^{\prime}}\right)=\left\langle A(\vec{x}, t) A\left(\overrightarrow{x^{\prime}}, t\right) \sin \left[\phi(\vec{x}, t)-\phi\left(\overrightarrow{x^{\prime}}, t\right)\right]\right\rangle, \\
& \bar{C}\left(\vec{x}, \overrightarrow{x^{\prime}}\right)=\left\langle A(\vec{x}, t) A\left(\overrightarrow{x^{\prime}}, t\right) \cos \left[\phi(\vec{x}, t)-\phi\left(\overrightarrow{x^{\prime}}, t\right)\right]\right\rangle,
\end{aligned}
$$

and 


$$
\begin{aligned}
D\left(\vec{x}, \overrightarrow{x^{\prime}}\right) & =\bar{C}\left(\vec{x}, \overrightarrow{x^{\prime}}\right) \cos \left[\theta(\vec{x})-\theta\left(\overrightarrow{x^{\prime}}\right)\right] \\
& +\bar{S}\left(\vec{x}, \overrightarrow{x^{\prime}}\right) \sin \left[\theta(\vec{x})-\theta\left(\overrightarrow{x^{\prime}}\right)\right] \quad\left(=\sqrt{\bar{C}^{2}+\bar{S}^{2}}\right) .
\end{aligned}
$$

Thus, Eqs. (21) and (22) become, respectively,

$$
\tan \left[\theta(\vec{x})-\theta\left(\overrightarrow{x^{\prime}}\right)\right]=\frac{\bar{S}\left(\vec{x}, \overrightarrow{x^{i}}\right)}{\overline{\bar{C}}\left(\vec{x}, \overrightarrow{x^{\prime}}\right)}
$$

and

$$
\int_{W(\vec{x})} W\left(\overrightarrow{x^{\prime}}\right) B\left(\overrightarrow{x^{\prime}}\right) D\left(\vec{x}, \vec{x}^{\prime}\right) d \vec{x}^{\prime}-\lambda B(\vec{x})=0
$$

Equations (26) and (27) constitute the requirements on the local oscillator pinase $\theta(\vec{x})$ and amplitude $B(\vec{x})$, respectively, to ensure the maximum average signal-to-noise ratio. The only properties of the received-signal beam appearing in these equations (namely, $\bar{C}$ and $\bar{S}$ ) are, respectively, the real and imaginary parts of $\left\langle U(\vec{x}, t) U^{*}\left(\vec{x}^{\prime}, t\right)\right\rangle$ which represents a two-point correlation on the incoming signal wavefront. Physically, $\bar{C}$ represents the average homodyne beat signal obtained by adding the signals $U(\vec{x}, t)$ and $U\left(\overrightarrow{x^{t}}, t\right)$ on the surface of a square law detector. Similarly, $\bar{S}$ represents the homodyne beat signal obtained by adding signals $U(\vec{x}, t)$ and $U\left(\vec{x}^{\prime}, t\right)$, the latter delayed by $\pi / 2$ radians, on the surface of a square law detector. A method for experimentally determining these two functions is given in Appendix $B$. 
Note that Eq. (26) determines only the difference in phase between pairs of points on the local oscillator wavefront; i. e., the shape of this wavefront. This is as it should be since adding a constant term $\theta_{0}$ to any $\theta(\vec{x})$ merely alters the time at which I achieves its peak value during one cycle of the beat frequency, but has no effect on $P$ nor $\langle P\rangle$. Thus, arbitrarily choosing the origin of coordinates as the phase reference point (i. e., $\theta(\overrightarrow{0})=0$ ) Eq. (26) determines $\theta(\vec{x})$ in the form

$$
\theta(\vec{x})=\tan ^{-1}\left\{\frac{\bar{S}(\vec{x}, \overrightarrow{0})}{\bar{C}(\vec{x}, \overrightarrow{0})}\right\}
$$

In this Chapter the equations which determine the optimum local oscillator wave function have been derived.

In the following Chapter, the optimum local oscillator wavefront will be determined for several simple examples, the solution for the case of an atmospherically distorted wavefront being deferred to Chapter V. 


\section{CHAPTER III \\ SOLUTION OF EQUATIONS FOR \\ SEVERAL SIMPLE EXAMPLES}

In this Chapter the optimum local oscillator wave function will be determined for two simple examples. In addition, the conditions under which the optimum local oscillator phase front is parallel to the average received-signal phase front will be determined.

For the case in which neither $A$ nor $\phi$ is a function of time, Eq. (28) should reduce to the well-known result that the local oscillator wavefront should be chosen parallel to the signal beam wavefront. Indeed, from Eqs. (23) and (24)

$$
\bar{S}(\vec{x}, 0)=A(\vec{x}) A(\overrightarrow{0}) \sin [\phi(\vec{x})-\phi(\overrightarrow{0)}]
$$

and

$$
\bar{C}(\vec{x}, 0)=A(\vec{x}) A(\overrightarrow{0}) \cos [\phi(\vec{x})-\phi(\overrightarrow{0})],
$$

and hence, Eq. (28) becomes

$$
e(\vec{x})=\tan ^{-1}\left\{\frac{\sin [\phi(\vec{x})-\phi(\overrightarrow{0})]}{\cos [\phi(\vec{x})-\phi(\overrightarrow{0})]}\right\}
$$

or

$$
\text { (31) } \quad \theta(\vec{x})=\phi(\vec{x})-\phi(\overrightarrow{0})
$$

as stated. 
To determine the corresponding amplitude shape, substitute Eq. (31) into Eq. (25) to obtain

$$
\begin{aligned}
D\left(\vec{x}, \overrightarrow{x^{\prime}}\right) & =A(\vec{x}) A\left(\vec{x}^{\prime}\right) \cos \left[\phi(\vec{x})-\phi\left(\vec{x}^{\prime}\right)\right] \cos \left[\phi(\vec{x})-\phi\left(\vec{x}^{\prime}\right)\right] \\
& +A(\vec{x}) A\left(\vec{x}^{\prime}\right) \sin \left[\phi(\vec{x})-\phi\left(\overrightarrow{x^{\prime}}\right)\right] \sin \left[\phi(\vec{x})-\phi\left(\overrightarrow{x^{\prime}}\right)\right]
\end{aligned}
$$

or

$$
D\left(\vec{x}, \vec{x}^{\prime}\right)=A(\vec{x}) A\left(\overrightarrow{x^{\prime}}\right)
$$

Subsituting this result into Eq. (27) gives, for the equation to be satisfied by $B(\vec{x})$,

$$
\int W(\vec{x}) W\left(\overrightarrow{x^{\prime}}\right) B\left(\vec{x}^{\prime}\right) A(\vec{x}) A\left(\overrightarrow{x^{\prime}}\right) d \vec{x}^{\prime}-\lambda B(\vec{x})=0
$$

or

$$
W(\vec{x}) A(\vec{x}) \int W\left(\overrightarrow{x^{\prime}}\right) B\left(\overrightarrow{x^{\prime}}\right) A\left(\vec{x}^{\prime}\right) d \overrightarrow{x^{\prime}}-\lambda B(\vec{x})=0
$$

But the integral in Eq. (34) is independent of $\vec{x}$ and hence its solution for $B(\vec{x})$ is, simply

$$
B(\vec{x})=\text { (const.) } A(\vec{x}) W(\vec{x})=C A(\vec{x}) W(\vec{x}) \text {, }
$$

where the constant may be determined from the condition of constant local oscillator power; that is, from Eq. (8)

$$
C^{2}=\frac{P_{L}}{\frac{1}{2} \eta \int W(\vec{x}) A^{2}(\vec{x}) d \vec{x}}
$$


Thus, as stated in the introduction, the maximum beat signal amplitude, for fixed local oscillator power, is obtained by choosing not only parallel wavefronts but also identical amplitude shapes for the received signal and local oscillator beams. Equation (35) is shown in Appendix I to be, simply, a statement of the Schwarz inequality applied to the ratio $P_{0} / P_{L}$.

Next, the condition for which the local oscillator phase front should be chosen parallel to the average received signal phase front is derived; that is, the condition for which $\theta(\vec{x})=\langle\phi(\vec{x}, t)-\phi(\overrightarrow{0}, t)\rangle$ is determined. Thus, from Eq. (28), it follows that this condition will obtain, provided

$$
\tan \langle\phi(\vec{x}, t)-\phi(\overrightarrow{0}, t)\rangle=\frac{\vec{S}(\vec{x}, \overrightarrow{0})}{\bar{C}(\vec{x}, \overrightarrow{0})}
$$

In order to determine the condition under which Eq. (36) holds, let

$$
\phi(\vec{x}, t)-\phi(\overrightarrow{0}, t)=\Delta \phi_{0}(\vec{x})+\alpha_{0}(\vec{x}, t)
$$

where $\alpha_{0}(\vec{x}, t)$ represents the fluctuations of $\phi(\vec{x}, t)-\phi(\overrightarrow{0}, t)$ about its average value

$$
\Delta \phi_{0}(\vec{x})=\langle\phi(\vec{x}, t)-\phi(\overrightarrow{0}, t)\rangle .
$$


Then, from Eqs. (23) and (24), Eq. (36) may be written

$$
\tan \Delta \phi_{0}(x)=\frac{\left\langle A(\vec{x}, t) A(\overrightarrow{0}, t) \sin \left[\Delta \phi_{0}(\vec{x})+\alpha_{o}(\vec{x}, t)\right]\right\rangle}{\left\langle A(\vec{x}, t) A(\overrightarrow{0}, t) \cos \left[\Delta \phi_{0}(\vec{x})+\alpha_{o}(\vec{x}, t)\right]>\right.}
$$

or

$$
\tan \Delta \phi_{0}(\vec{x})=
$$

$<A(\vec{x}, t) A(\overrightarrow{0}, t) \dot{c o s} \alpha_{O}(\vec{x}, t)>\sin \Delta \phi_{O}(\vec{x})+<A(\vec{x}, t) A(\overrightarrow{0}, t) \sin \alpha_{O}(\vec{x}, t)>\cos \Delta \phi_{0}(\vec{x})$

$\left\langle A(\vec{x}, t) A(\overrightarrow{0}, t) \cos \alpha_{o}(\vec{x}, t)>\cos \Delta \phi_{0}(\vec{x})-<A(\vec{x}, t) A(\overrightarrow{0}, t) \sin \alpha_{o}(\vec{x}, t)>\sin \Delta \phi_{o}(\vec{x})\right.$

W riting

$$
\tan \Delta \phi_{0}(\vec{x})=\frac{\sin \Delta \phi_{0}(\vec{x})}{\cos \Delta \phi_{0}(\vec{x})}
$$

and cross-multiplying, Eq. (40) yields

$$
<A(\vec{x}, t) A(\overrightarrow{0}, t) \sin \alpha_{o}(\vec{x}, t)>\left[\sin ^{2} \Delta \phi_{0}(\vec{x})+\cos ^{2} \Delta \phi_{0}(\vec{x})\right]=0
$$

or

$$
\left\langle A(\vec{x}, t) A(\overrightarrow{0}, t) \sin \alpha_{0}(\vec{x}, t)\right\rangle=0
$$

Equation (41), then, is the condition on the received signal wave function for which the optimum local oscillator phase front is parallel to the average received-signal phase front. This condition obtains, for example, when the fluctuations in the phase difference $\phi(\vec{x}, t)-\phi(\overrightarrow{0}, t)$ is symmetric about its average value; i. e., when the fluctuations in $\alpha_{o}(\vec{x}, t)$ are symmetric about zero, and the fluctuations of the received 
signal amplitude product $A(\vec{x}, t) A(0, t)$ are independent of the fluctuations in the phase difference between these points. That is, the latter of these conditions implies that

$$
\left\langle\mathrm{A}(\overrightarrow{\mathrm{x}}, \mathrm{t}) \mathrm{A}(\overrightarrow{0, t}) \sin \alpha_{0}(\vec{x}, t)\right\rangle=\langle\mathrm{A}(\overrightarrow{\mathrm{x}}, \mathrm{t}) \mathrm{A}(\overrightarrow{0}, \mathrm{t})\rangle\left\langle\sin \alpha_{0}(\overrightarrow{\mathrm{x}}, \mathrm{t})\right\rangle
$$

while the former condition imples that $\left\langle\sin \alpha_{0}(\vec{x}, t)\right\rangle=0$ and hence Eq. (4l) is satisfied.

The results of the following example will serve to illustrate the basic conditions for which there exists an aperture size above which there is little or no increase in the arerage signal-to-noise ratio. In addition, a simple extension of this example will, in Chapter IV, provide the basis for an alternative derivation of the equations defining the optimum local oscillator wave function. Thus, consider the following:

Let the receiver aperture be divided into $N$ equal area regions as shown in Fig. 2. Let both the amplitude, $A_{i}(t)$ and the phase, $\phi_{i}(t)$, of the received signal be independent of position over each region $S_{i} ; i=1,2, \ldots, N$. Assume, for now, that the optimum (fixed) local oscillator beam will also have uniform amplitude, $B_{i}$, and phase $\theta_{i}$, over each region $S_{i} ;=1,2, \ldots, N$. Then, with $U_{i}(t)=$ $A_{i} e^{j \phi i}$ and $v_{i}=B_{i} e^{j \theta_{i}}$, it follows, from Eqs. (7) and (8), that 


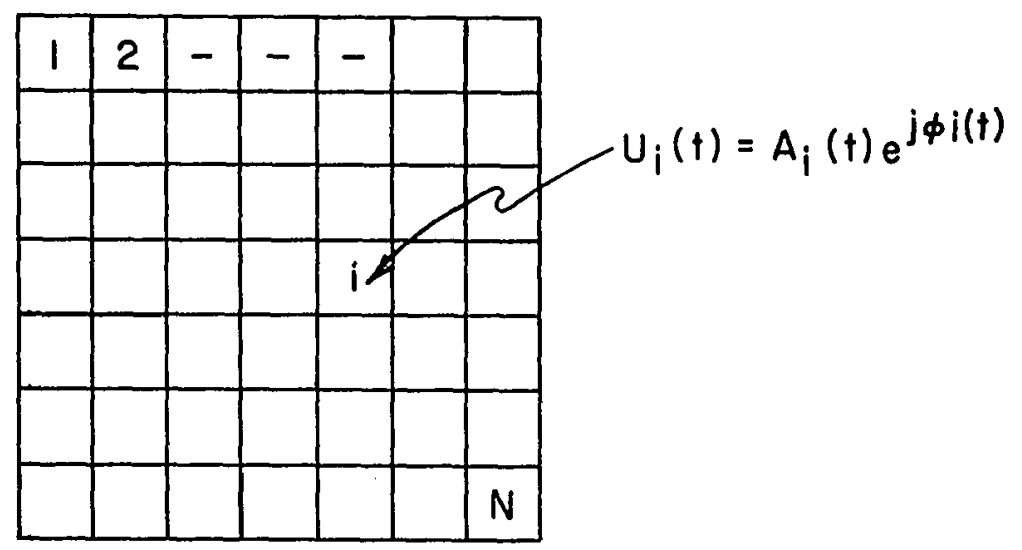

Fig. 2--Received signal (for the example used in Chapter III).

(42)

$$
\frac{P_{O}}{P_{L}}=\frac{\frac{1}{2} \eta^{2}(\Delta S)^{2} \sum_{i, j=1}^{N} v_{i}^{*} v_{j}\left\langle U_{i} U_{j}^{*}\right\rangle}{\frac{1}{2} \eta(\Delta S) \sum_{i=1}^{N}\left|v_{i}\right|^{2}}
$$

or

(43)

$$
\frac{P_{0}}{P_{L}}=\eta(\Delta S) \frac{\sum_{i=1}^{N} B_{i}^{2}\left\langle A_{i}^{2}\right\rangle+2 \sum_{\substack{i, j=1 \\ i<j}}^{N} v_{i}^{*} v_{j} M_{i j}}{\sum_{i=1}^{N} B_{i}^{2}}
$$

where $\Delta S$ is the area of the region $S_{i}$ and

(44)

$$
M_{i j}=\left\langle U_{i} U_{j}^{* *}\right\rangle
$$


Consider first the case where the fluctuations in the received signal are absent. Then, with $\phi_{i}=\phi_{0}$ and $A_{i}=A_{0}$ for all $i$, it follows that $M_{i j}=A_{0}^{2}$ and hence, with $\theta_{i}=\theta_{0}$ and $B_{i}=B_{0}$ for all $i$,

$$
\frac{P_{0}}{P_{I}}=\eta A_{0}^{2} \Delta S \frac{N B_{0}^{2}+2 B_{0}^{2} \frac{N(N-1)}{2}}{N B_{0}^{2}}
$$

or

$$
\frac{P_{O}}{P_{L}}=\eta A_{O}^{2}(N \Delta S)
$$

That is, as shown previously, the signal-to-noise ratio is directly proportional to the collected signal power, i. e. , to $\frac{1}{2} \eta A_{0}^{2}$ (aperture area) $=\frac{1}{2} \eta A^{2}(N \Delta S)$. [NOTE: It is a simple matter to show, as was done previously, that for any choice for $B_{i}$ and $\theta_{i}$ other than $B_{i}=B_{0}$ and $\theta_{i}=\theta_{0}$, the signal-to-noise ratio is less than that given by Eq. (46).]

On the other hand, for the very special case where the signal correlation between any two distinct regions is zero, i. e., $M_{i j}=0$ for $i \neq j$, it follows that, with $\left\langle A_{i}^{2}\right\rangle=A_{0}^{2}$,

or

$$
\frac{P_{0}}{P_{L}}=\eta A_{0}^{2}(\Delta S) \frac{\sum_{i=1}^{N} B_{i}^{2}}{\sum_{i=1}^{N} B_{i}^{2}}
$$

$$
=\eta A_{0}^{2}(\Delta S)
$$


which is identical to the signal-to-noise ratio obtained from one area alone. It is important to note here that even in this extreme case the average beat signal power increases directly with $N$, for $B_{i}=B_{0}$, but so too does the noise power (which is proportional to $P_{L}$ ) with the result that the signal-to-noise ratio is independent of $\mathrm{N}$.

In a more general case, for example where the correlation between two regions is a decreasing function of their separation, the signal-to-noise ratio lies somewhere between those two extremes. When $B_{i}=$ constant, the important point to note is that as $N$ increases the sum in E.q. (45) deviates more and more from its maximum value, $N(N-1) / 2$, and hence the rate of growth of $P_{O} / P_{L}$, for a unit increase in $N$, decreases with increasing $N$. This is, of course, the basic result obtained by Fried[ 1] which indicates the existence of a minimum aperture size, above which the increase in signal-to-noise ratio is "small". With reference to this last case, the problem under consideration here would be to determine how the $B_{i}{ }^{\prime} s$ and $\theta_{i}{ }^{\prime} s$ should be chosen so as to maximize the average signal-to-noise ratio for a given size aperture.

In this Chapter the optimum local oscillator beam was determined for several simple examples. The next Chapter gives an alternative derivation of the results of Chapter II. 


\section{CHAPTER IV \\ ALTERNATIVE DERIVATION OF INTEGRAL EQUATION DETERMINING OPTIMUM LOCAL OSCIL LATOR WAVE FUNCTION AND PHYSICAL INTERPRETATION OF RESULTS}

In this Chapter the integral equation which determines the optimum local oscillator wave function is derived as a consequence of the Karhunen-Loéve Theorem[12]. This derivation allows for both physical interpretations and additional consequences of the results of

Chapter II. These include: a measure of the amount of turbulent degradation (based on relative sizes of the eigenvalues); and a means for separating a distorted signal beam into a set of statistically orthogonal signals.

\section{A. Introduction}

A way of introducing the subject clearly is to first consider a simple extension of the last example of Chapter III. Consider again that the receiver aperture can be divided into $N$ equal a rea regions over each of which both the amplitude, $A_{i}(t)$, and the phase, $\phi_{i}(t)$, of the received signal, $U(\vec{x}, t) W(\vec{x})$ are independent of position. Again, let the mutual coherence of the signals at any two distinct locations be zero. This time, however, let the average intensity, 
$\left\langle A_{i}^{2}(t)\right\rangle$, of the signal at location $i$ depend on $i$ Then, from Eq. (43)

$$
\frac{P_{0}}{P_{L}}=\eta \Delta S \frac{\sum_{i=1}^{N}\left\langle A_{i}^{2}(t)\right\rangle B_{i}^{2}}{\sum_{i=1}^{N} B_{i}^{2}}
$$

For the case where $\left\langle A_{i}^{2}\right\rangle$ was independent of location (the case treated previously), the maximum signal-to-noise ratio was shown to be equivalent to that obtained from a single area $S_{i}$. Which area $S_{i}$ was chosen made no difference since the average received optical signal power was the same for all of them. For the present example it is reasonable to expect again that the maximum signal-to-noise ratio will be equal to that obtainable from a single area. This time, however, one should expect the area to be that one for which the average optical signal power is largest. This conclusion is correct and can be formally verified as follows:

The $B_{i}{ }^{\prime} s$ which maximize $P_{0} / P_{L}$ are determined by setting

$$
\frac{\partial\left(\mathrm{P}_{\mathrm{O}} / \mathrm{P}_{\mathrm{L}}\right)}{\partial \mathrm{B}_{\mathrm{i}}}=0 \quad ; \quad \mathrm{i}=1,2, \ldots, \mathrm{N}
$$


Thus, from Eq. (49)

$$
\begin{aligned}
& \frac{\partial\left(P_{0} / P_{L}\right)}{\partial B_{j}}= \\
& \eta \Delta S \frac{\left(\sum_{i=1}^{N} B_{i}^{2}\right)\left(2 B_{j}<A_{j}^{2}>\right)-\left(\sum_{i=1}^{N}<A_{i}^{2}>B_{i}^{2}\right)\left(2 B_{j}\right)}{\left(\sum_{i=1}^{N} B_{i}^{2}\right)^{2}}
\end{aligned}
$$

or

$$
\begin{gathered}
\frac{\partial\left(P_{O} / P_{L}\right)}{\partial B_{j}}= \\
\left\{\frac{\eta \Delta S}{\left(\sum_{i=1}^{N} B_{i}^{2}\right)^{2}}\right\} \quad 2 B_{j}\left\{\sum_{i=1}^{N} B_{i}^{2}\left[\left\langle A_{j}^{2}\right\rangle-\left\langle A_{i}^{2}\right\rangle\right]\right\}
\end{gathered}
$$

It is easy to see that a solution of the equation, $\frac{\partial\left(P_{0} / P_{L}\right)}{\partial B_{j}}=0$; $j=1,2, \ldots, N$, is obtained by choosing all of the $B_{i}{ }^{\prime}$ s equal to zero except one. Thus, let

$$
B_{i}=\left\{\begin{array}{l}
B_{q} \neq 0 \text { for } i=q \\
0 \text { for } i \neq q
\end{array} \quad ; i=1,2, \ldots, N .\right.
$$

To see that this is indeed a solution note that for $j \neq q$ in Eq. (52), the factor $2 B_{j}$ is zero and hence, $\frac{\partial\left(P_{0} / P_{L}\right)}{\partial B_{j}}=0$. On the othet hand, for $j=q$ every term in the third factor on the right hand side of Eq. (52) 
is zero. This is true since $B_{i}^{2}=0$ for $i \neq j=q$ and $\left\langle A_{i}^{2}\right\rangle-\left\langle A_{j}^{2}\right\rangle \equiv 0$ for $i=j$. Hence, $\frac{\partial\left(P_{0} / P_{L}\right)}{\partial B_{q}}=0$.

The corresponding ratio of $P_{O}$ to $P_{L}$ is

$$
\left.\frac{P_{O}}{P_{L}}=\eta \Delta S<A_{q}^{2}\right\rangle
$$

and this is clearly a true maximum for that $q$ for which $\left\langle A_{q}^{2}\right\rangle \geq\left\langle A_{i}^{2}\right\rangle$ $i=1,2, \ldots, N$.

Thus, as expected, the maximum average signal-to-noise ratio is equal to that obtained by heterodyning with a single area - namely, that particular area for which the average received optical signal power is largest.

It is important here to extract the essential features of the above example. They are:

(1) The received signal $U(\vec{x}, t) W(\vec{x})$ may be viewed as a sum of $N$ time independent signals, $\mathrm{p}_{i}(\overrightarrow{\mathrm{x}})$, with ( random) "complex amplitudes" $U_{i}(t)$. In this example $p_{i}(\vec{x})$ is a pulse type function with amplitude one over the region $S_{i}$ and zero amplitude elsewhere. Mathematically, the essential property of the pulses, $p_{i}(\vec{x})$, is not that they are physically separated in space, but rather that they are mutually or thogonal over the receiver aperture. That is 


$$
\int p_{i}(\vec{x}) p_{j}(\vec{x})=\left\{\begin{array}{l}
1 \text { if } i=j \\
0 \text { if } i \neq j
\end{array}\right.
$$

(2) The "amplitudes", $U_{i}(t)$, in this series representation of the random signal are mutually or thogonal, i. e. ,

$$
\left\langle U_{i} U_{j}^{*}\right\rangle=\left\{\begin{array}{cc}
\left\langle A_{i}^{2}\right\rangle & \text { if } i=j \\
0 & \text { if } i \neq j
\end{array}\right.
$$

( If $\left\langle U_{i}\right\rangle=0$ for all $i$, then the orthogonality is equivalent to uncorrelatedness.)

(3) The optimum local oscillator beam is determined by that pulse $p_{q}(\vec{x})$ which, in the series representation of $U(\vec{x}, t) W(\vec{x})$, has associated with it the largest average optical signal power.

It follows then that if the received signal $U(\vec{x}, t) W(\vec{x})$, can be represented in terms of a series of spatially orthogonal signals, $\left\{\Phi_{n}(\vec{x})\right\}$, with orthogonal coefficients, $\left\{U_{n}(t)\right\}$, then the optimum (fixed) local oscillator beam is proportional to $\Phi_{q}(\vec{x})$, where $\left\langle\left|\mathrm{U}_{\mathrm{q}}\right|^{2}\right\rangle \geq\langle| \mathrm{U}_{\mathrm{i}} \beta^{2}$. This argument assumes, of course, that the optimum local oscillator beam can be expanded in terms of the set $\left\{\Phi_{n}(\vec{x})\right\}$. . 
B. Karhunen-Loéve Theorem

The results of the previous section lead one naturally to ask if any received signal can be expressed in terms of a series of spatially orthogonal functions with "statistically" orthogonal coefficients. According to the Karhunen-Loe've theorem[12] the answer is yes! provided only that the received signal possesses a continuous mutual coherence function. A statement of this theorem follows[24]: There exists a series, $\sum U_{n}(t) \phi_{n}(\vec{x})$, possessing the properties that

$$
\int W(\vec{x}) \phi_{n}(\vec{x}) \phi_{m}^{*}(\vec{x}) d \vec{x}=\delta_{m n}
$$

and

$$
\left\langle U_{n}(t) U_{m}^{*}(t)\right\rangle=\left\langle\left|U_{n}(t)\right|^{2}\right\rangle \delta_{m n}
$$

which is convergent in a "mean square sense" uniformly to $U(\vec{x}, t) W(\vec{x})$ if, and only if, the numbers $\left\langle\left|U_{n}(t)\right|^{2}\right\rangle$ and the corresponding functions $\phi_{n}(\vec{x})$ are, respectively, the eigenvalues, $\lambda$, and the orthonormal eigenfunctions, $\phi(\vec{x})$, of the integral equation

$$
\int M_{u}\left(\vec{x}, \overrightarrow{x^{\prime}}\right) \phi\left(\overrightarrow{x^{\prime}}\right) d \overrightarrow{x^{\prime}}=\lambda \phi(\vec{x})
$$

where 


$$
M_{u}\left(\vec{x}, \overrightarrow{x^{\prime}}\right)=\left\langle U(\vec{x}, t) W(\vec{x}) U^{* *}\left(\overrightarrow{x^{\prime}}, t\right) W\left(\overrightarrow{x^{\prime}}\right)\right\rangle
$$

Proof: Suppose $U(\vec{x}, t) W(\vec{x})$ has the asserted decomposition. Let $\vec{x}$ and $\overrightarrow{x^{\prime}}$ vary over $W(\vec{x})$, and

$$
\mathrm{Z}_{N}(\vec{x}, t)=\sum_{i=1}^{N} U_{i}(t) \phi_{i}(\vec{x})
$$

be the $N^{\text {th }}$ partial sum of the series. Since $Z_{N}(t)$ is convergent in the mean square sense uniformly to $U(\vec{x}, t) W(\vec{x})$, i. e. ,

$$
<\left|U(\vec{x}, t) W(\vec{x})-Z_{N}(\vec{x}, t)\right|^{2}>\rightarrow 0 \text { uniformly as } N \text { increases, }
$$

it follows that

$$
\left\langle Z_{N}(\vec{x}, t) Z_{N}^{*}\left(\overrightarrow{x^{\prime}}, t\right)\right\rangle \rightarrow M_{u}\left(\vec{x}, \overrightarrow{x^{\prime}}\right) \text { uniformly as } N \text { increases. }
$$

But

$$
\left\langle Z_{N}(\vec{x}, t) Z_{N}^{*}\left(\overrightarrow{x^{\prime}}, t\right)\right\rangle=\sum_{j=1}^{N}\left\langle\left|U_{j}(t)\right|^{2}\right\rangle \phi_{j}(\vec{x}) \phi_{j}^{*}\left(\overrightarrow{x^{\prime}}\right)
$$

and hence

$$
\int M_{u}\left(\vec{x}, \overrightarrow{x^{\prime}}\right) \phi_{n}\left(\overrightarrow{x^{\prime}}\right) d \overrightarrow{x^{\prime}}=\left\langle\left|U_{n}(t)\right|^{2}\right\rangle \phi_{n}(\vec{x})
$$

and the "only if" part of the theorem is confirmed. To demonstrate the "if" part of the theorem note first that 
(1) From the fact that $M_{\mathrm{u}}(\vec{x}, \vec{x})$ is defined, continuous and symmetrical [i.e., $M_{u}\left(\vec{x}, \overrightarrow{x^{\prime}}\right)=M_{u}^{*}\left(\overrightarrow{x^{\prime}}, \vec{x}\right)$ ] over $W(\vec{x})$ if follows[25] that: The integral equation, Eq. (57), possesses a set (finite or denumerably infinite) of eigenvalues to each of which there corresponds a finite set (which may always be assumed to be an orthonormal set) of linearly independent eigenfunctions. The eigenfunctions corresponding to distinct eigenvalues a re orthogonal.

(2) $M_{u}\left(\vec{x}, \vec{x}^{\prime}\right)$, being a mutual coherence function, is a nonnegative definite type function, i. e., $\iint M_{u}\left(\vec{x}, \overrightarrow{x^{\prime}}\right) V^{* *}(\vec{x}) V\left(\overrightarrow{x^{\prime}}\right) d \vec{x} d \vec{x}^{\prime}$ $=\left\langle\left|\int U(\vec{x}, t) W(\vec{x}) V^{*}(\vec{x}) d \vec{x}\right|^{2}\right\rangle \geq 0$ for all $V(\vec{x})$. For this case Mercer's theorem[25] states that, with each eigenvalue (necessarily positive) listed as many times as its multiplicity ( and with distinct indices) and in the order $\lambda_{1} \geq \lambda_{2} \geq \lambda_{3} \cdots$,

$$
M_{u}\left(\vec{x}, \vec{x}^{\prime}\right)=\sum \lambda_{i} \phi_{i}(\vec{x}) \phi_{i}^{* *}\left(\vec{x}^{\prime}\right)
$$

where the convergence is uniform. [If an eigenvalue has multiplicity greater than one, the set $\left\{\phi_{\mathrm{n}}(\vec{x})\right\}$ is not uniquely defined. But $\mathrm{M}_{\mathrm{u}}\left(\overrightarrow{\mathrm{x}}, \overrightarrow{\mathrm{x}^{\prime}}\right)$ is, as distinct sets lead only to a "regrouping" of the terms in a given series. ] 
In order to construct the desired series for $U(\vec{x}, t) W(\vec{x})$ let

$$
U_{n}(t)=\int U(\vec{x}, t) W(\vec{x}) \phi_{n}^{*}(\vec{x}) d \vec{x}
$$

It follows that $U_{n}(t)$ exists and

$$
\left\langle U_{n}(t) U_{m}^{*}(t)>=\lambda_{n} \delta_{n m}\right.
$$

and hence Eq. ( 56 ) is satisfied. In addition Eq. (55) is satisfied because the $\phi_{n}(\vec{x})$ were choosen to be orthonormal. Finally, the convergence of the series $\sum U_{n}(t) \phi_{n}(\vec{x})$ in the mean square sense uniformly to $U(\vec{x}, t) W(\vec{x})$ follows by Mercer's theorem. Q.E.D.

The above characterization of the signal $U(\vec{x}, t) W(\vec{x})$ is now sufficient to deduce that the optimum local oscillator wave function, $V(\vec{x})$, must be a solution of the integral equation, Eq. (57), corresponding to the maximum eigenvalue. To show this, let $V(\vec{x})$ be an arbitrary, square integrable local oscillator wave function and note from Eq. (9) that

$$
P_{0}=\frac{1}{2} \eta^{2} \iint M_{u}\left(\vec{x}, \overrightarrow{x^{\prime}}\right) V^{* *}(\vec{x}) V\left(\overrightarrow{x^{\prime}}\right) d \vec{x} d \overrightarrow{x^{\prime}}
$$

But, from Eq. (62)

$$
\begin{aligned}
\int M_{u}\left(\vec{x}, \overrightarrow{x^{\prime}}\right) V\left(\overrightarrow{x^{\prime}}\right) \overrightarrow{d x^{\prime}} & =\int \sum \lambda_{i} \phi_{i}(x) \phi_{i}^{*}\left(\overrightarrow{x^{\prime}}\right) v\left(\overrightarrow{x^{\prime}}\right) d \overrightarrow{x^{\prime}} \\
& =\sum \lambda_{i} V_{i} \phi_{i}(\vec{x})
\end{aligned}
$$


where

$$
V_{i}=\int V\left(\vec{x}^{\prime}\right) \phi_{i}^{*}\left(\vec{x}^{\prime}\right) d \overrightarrow{x^{\prime}}
$$

and the convergence is uniform. It follows then that

$$
P_{0}=\frac{1}{2} \eta^{2} \sum \lambda_{i}\left|v_{i}\right|^{2}
$$

Noting[27] that $V(\vec{x})$ admits the expansion, convergent in the mean,

$$
V(\vec{x}) \sim \sum v_{i} \phi_{i}(\vec{x})+h(\vec{x})
$$

where $h(\vec{x})$ is some function dependent on $V(\vec{x})$ and orthogonal to all the $\phi_{i}(\vec{x}) \quad\left[h(\vec{x})=0\right.$ if the set $\left\{\phi_{n}(\vec{x})\right\}$ is complete ], it follows that

$$
P_{L}=\frac{1}{2} \eta \int V(\vec{x}) V^{*}\left(\overrightarrow{x^{\prime}}\right) d \vec{x}=\sum\left|v_{i}\right|^{2}+\int|h(\vec{x})|^{2} d \vec{x}
$$

and hence

$$
\frac{P_{0}}{P_{L}}=\eta \frac{\sum \lambda_{i}\left|v_{i}\right|^{2}}{\sum\left|v_{i}\right|^{2}+\int|h(\vec{x})|^{2} d \vec{x}}
$$

Finally, since the $V_{i}$ are independent of $h(\vec{x})$, it follows ( see pp. 42-43) that $P_{o} / P_{I}$ is a maximum when $h(\vec{x})=0$ and all the $V_{i}$, except $V_{q}$, are equal to zero, where $\mathrm{q}$ is such that

$$
\lambda_{\mathrm{q}}=\left\langle\left|\mathrm{U}_{\mathrm{q}}(\mathrm{t})\right|^{2}\right\rangle \geq\left\langle\left|\mathrm{U}_{\mathrm{i}}(\mathrm{t})\right|^{2}\right\rangle=\lambda_{\mathrm{i}}
$$


Therefore, an optimum local oscillator wave function is

$$
v(\vec{x})=v_{q} \phi_{q}(\vec{x})
$$

and the corresponding maximum average signal-to-noise ratio is

$$
\left(\frac{\mathrm{S}}{N}\right)_{\max }=\frac{1}{2 e}\left(\frac{P_{0}}{P_{L}}\right)_{\max }=\frac{\eta}{2 e}(\lambda)_{\max }
$$

This completes the determination of the optimum local oscillator wave function. The results, obtained previously in Chapter I, have now been shown to be rather simple consequences of the Karhunen-Loéve theorem.

\section{Interpretation of Eigenvalues}

The above derivation allows for an easily obtainable physical interpretation, and then further consequences, of the eigenvalues of the integral equation. Eq. (57). Thus, note that the average total received optical power,

$$
\left\langle\mathrm{P}_{\mathrm{s}}\right\rangle \triangleq \int\left\langle\mathrm{U}(\overrightarrow{\mathrm{x}}, \mathrm{t}) \mathrm{U}^{*}(\overrightarrow{\mathrm{x}}, \mathrm{t})\right\rangle \mathrm{W}(\overrightarrow{\mathrm{x}}) \mathrm{W}(\overrightarrow{\mathrm{x}}) \mathrm{d} \overrightarrow{\mathrm{x}},
$$

is, from Eqs. (62) and (64), given by

$$
\left\langle\mathrm{P}_{\mathrm{s}}\right\rangle=\sum\left\langle\left|\mathrm{U}_{\mathrm{n}}(t)\right|^{2}\right\rangle=\sum \lambda_{\mathrm{n}}
$$

Two important consequences of this result will now be derived. The first will lead to the conclusion, obtained previously, that in the absence of any fluctuations in the "shape" of the received signal the optimum local oscillator beam has the same "shape" as that of the 
received signal. The second result concerns a measure of the effect of the wavefront distortion based on the relative sizes of the eigenvalues $\lambda_{n}$.

Thus, it follows from Eq. (76) that for a given magnitude of $\left\langle P_{s}\right\rangle$, the maximum obtainable average signal-to-noise ratio $\left(\sim \lambda_{\max }\right)$ reaches its largest possible value when all $\lambda_{n}$, except one (any one) arezero. Then $\lambda_{\max }=\left\langle P_{S}\right\rangle$ and the average signal-tonoise ratio is proportional to the average collected optical power. Suppose then that $\lambda_{q}$ is the only non-zero value of $\lambda$. Then, from Eq. (60) $U_{q}$ is (at least with probability one) the only non-zero coefficient in the Karhunen-Loéve series for $U(\vec{x}, t) W(\vec{x})$. Thus, for this case

$$
U(\vec{x}, t) W(\vec{x})=U_{q}(t) \Phi_{q}(\vec{x})
$$

But it has already been shown by Eq. (73) that the optimum local os cillator wave function is,

$$
V(\vec{x})=V_{q} \Phi_{q}(\vec{x})
$$

This is, of course, the result obtained previously, although here in a somewhat more general form. Stated precisely, the result is: If the received signal is the product of a function of position only, times 
a function of time only, then the "complex wavefronts" of the received signal and local oscillator must be "parallel" over the detector surface.

The second interpretation of Eq. (76) is obtained by noting that, again for fixed $\left\langle P_{S}\right\rangle$, the maximum obtainable average signal-to-noise ratio has its minimum possible value when all $\lambda_{\mathrm{n}}$ have precisely the same value. Thus, suppose that the turbulence "scatters" the transmitted signal into, say $N$ modes, $\Phi_{1}, \Phi_{2}, \cdots, \Phi_{N}$ each pode possessing, on the average, the same optical signal power, i. e.,

$$
\left\langle\left|U_{1}\right|^{2}\right\rangle=\left\langle\left|U_{2}\right|^{2}\right\rangle=\ldots=\left\langle\left|U_{N}\right|^{2}\right\rangle
$$

or

$$
\lambda_{1}=\lambda_{2}=\cdots=\lambda_{N}
$$

Then

$$
\lambda_{\max }=\frac{<\mathrm{P}_{\mathrm{L}}>}{\mathrm{N}}
$$

and hence, the average signal-to-noise ratio $\left(\sim \lambda_{\max }\right)$ approaches zero as $\mathrm{N}$ becomes large.

It is apparent now, that the ratio of $\lambda_{\max }$ to $\sum_{n} \lambda_{n}$ affords a measure of the amount of "degradation" introduced by the turbulence. Indeed, the ratio

$$
y=\frac{\lambda_{\max }}{\sum_{n} \lambda_{n}}
$$


gives the factor by which the maximum possible average signal-tonoise ratio is reduced from its value in the absence of fluctuations in the "shape" of the received beam.

\section{Optical System}

To conclude this Chapter, consider finally the possibility that there exists an optical system which could spatially separate the light associated with the signals $U_{n}(t) \Phi_{n}(\vec{x})$ in the Karhunen-Loéve series expansion of the received $\operatorname{signal} U(\vec{x}, t) W(\vec{x})$. If such a system existed then the processed signal, say $\mathrm{Z}(\overrightarrow{\mathrm{y}}, \mathrm{t})$, would have precisely the particular form treated in the introductory example of this Chapter (see Fig. 3).

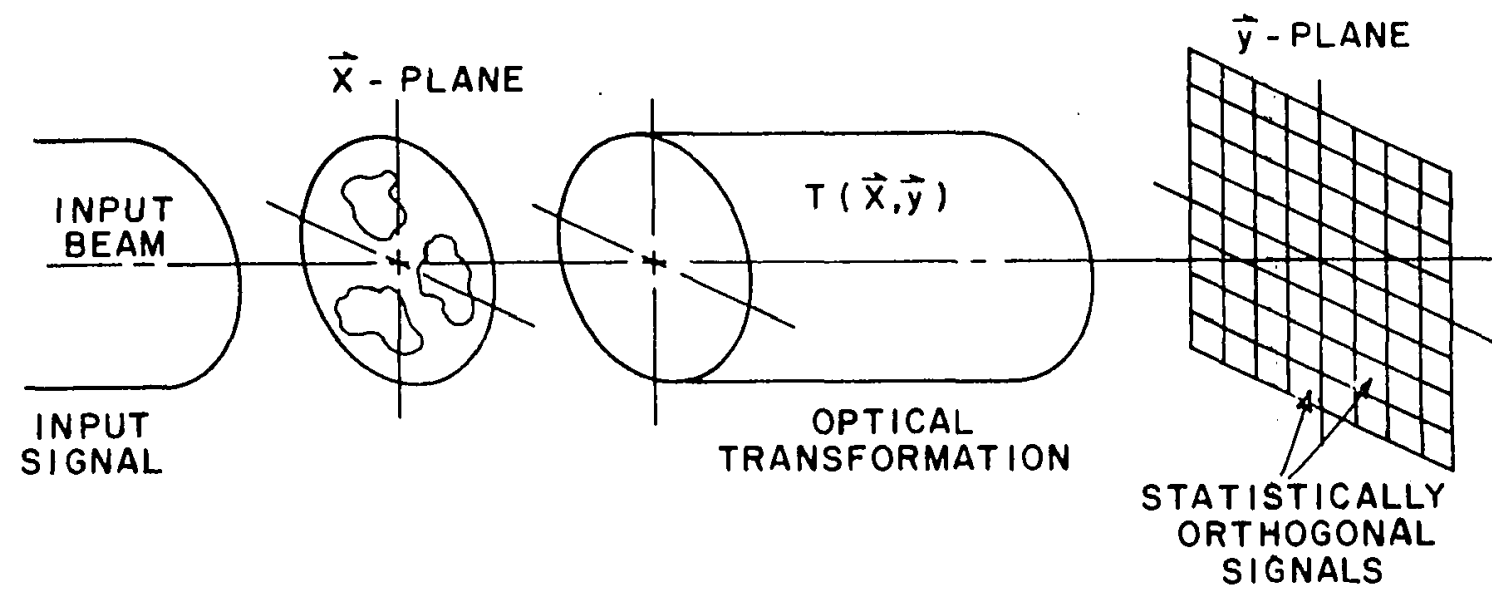

Fig. 3--Illustration of optical system described in Chapter IV. 
Thus, let us attempt to find the transfer function of an optical system which has the property that if $U(\vec{x}, t) W(\vec{x})$ is the input signal, then the output signal is

$$
Z(\vec{y}, t)=\sum_{n=1}^{\infty} U_{n}(t) \frac{1}{\sqrt{\Delta S_{n}}} p\left(y-y_{n}\right)
$$

where

$$
p\left(y-y_{n}\right)=\left\{\begin{array}{l}
1 \text { if } y \in \Delta S_{n} \\
0 \text { otherwise }
\end{array}\right.
$$

Thus, at location $\vec{y}=\overrightarrow{y_{n}}$

$$
\mathrm{Z}\left(\overrightarrow{\mathrm{y}}_{\mathrm{n}}, t\right)=\frac{1}{\sqrt{\Delta S_{n}}} U_{n}(t)
$$

where $\Delta S_{n}$ is the area occupied by the signal $Z\left(\overrightarrow{y_{n}}, t\right)$.

Assume that the optical system is a linear one with transfer function $T\left(\vec{x}, \vec{y}_{n}\right)$ so that

$$
Z\left(\overrightarrow{y_{n}}, t\right)=\int_{\int} W(\vec{x}) U(\vec{x}, t) T\left(\vec{x}, \overrightarrow{y_{n}}\right) d \vec{x}
$$

At this point either of two procedures may be used to determine $T\left(\vec{x}, \vec{y}_{n}\right)$. The first, and simplest, is to note that the coefficients, $U_{n}(t)$, in the Karhunen-Loe've series representation of $U(\vec{x}, t) W(\vec{x})$ are, from Eqs. ( 56) and (55), given by

$$
U_{n}(t)=\int_{W}(\vec{x}) U(\vec{x}, t) \Phi_{n}(\vec{x}) d \vec{x}
$$


This follows by multiplying both sides of Eq. (56) by $\Phi_{n}(\vec{x})$, integrating, and then making use of the orthogonality property of the functions $\left\{\Phi_{n}(\vec{x})\right\}$ as given by Eq. (55).

Upon comparing Eqs. (85), (86), and (87) it follows that

$$
T\left(\vec{x}, \overrightarrow{y_{n}}\right)=\frac{1}{\sqrt{\Delta S_{n}}} \Phi_{n}(\vec{x})
$$

An alternative, and more complicated, procedure for deriving the form of $T\left(\vec{x}, \overrightarrow{y_{n}}\right)$, which really assumes no knowledge of the KarhunenLoeve theorem, is to note that what is really being asked of the optical system is that it endow the processed signal, $Z(\vec{y}, t)$, with the property that the signals at any two distinct locations be orthogonal, i. e. ,

$$
\left\langle\mathrm{Z}\left(\overrightarrow{\mathrm{y}}_{\mathrm{n}}, t\right) \mathrm{z}^{*}\left(\overrightarrow{\mathrm{y}}_{\mathrm{m}}, t\right)\right\rangle=\left\{\begin{array}{l}
\frac{1}{\Delta \mathrm{S}_{\mathrm{n}}}<\left|\mathrm{U}_{\mathrm{n}}(\mathrm{t})\right|^{2}> \\
0 \text { if } \mathrm{m} \neq \mathrm{n}
\end{array}\right.
$$

In addition, note that the transformation $T\left(\vec{x}, \overrightarrow{y_{n}}\right)$ must be a unitary, or energy conserving, transformation. This follows since $U(\vec{x}, t) W(\vec{x})$ and $Z(\vec{y}, t)$, being but two representations of the same signal, must have the same energy. A more detailed discussion of this, unitary property, is given in Chapter VI.

The above characterization of the transformation $T\left(\vec{x}, \overrightarrow{y_{n}}\right)$ is sufficient to now deduce (by substituting Eq. (86) into Eq. (89), 
multiplying both sides by $\sum_{n=1}^{\infty} T\left(\vec{x}, \vec{y}_{m}\right) \Delta S_{m}$, and making use of the unitary property of $T)$ that $T\left(\vec{x}, \overrightarrow{y_{n}}\right)$ must satisfy the integral equation

$$
\int M_{u}\left(\vec{x}, \overrightarrow{x^{1}}\right) T\left(\overrightarrow{x^{1}}, \overrightarrow{y_{n}}\right) d \overrightarrow{x^{i}}=\left\langle\left|U_{n}\right|^{2}>T\left(\vec{x}, \overrightarrow{y_{n}}\right)\right.
$$

It follows then, upon comparing Eqs. (90) and (58), that

$$
T\left(\vec{x}, \vec{y}_{n}\right)=(\text { const. }) \Phi_{n}(\vec{x})=\frac{1}{\sqrt{\Delta S_{n}}} \Phi_{n}(\vec{x})
$$

which is precisely the result obtained by method 1.

This completes the alternative derivation, based on the KarhunenLoeve theorem, of the equations which determine the optimum local oscillator wave function. This derivation was shown to lead to previous results; to a measure of the turbulent degradation based on the relative sizes of the eigenvalues; and to the possibility of separating a distorted beam into a set of statistically orthogonal signals. An additional consequence of these results, which offers a possible method for determining the variance of the beat signal power, will be discussed in Chapter VI. In the following chapter the optimum local oscillator beam is determined explicitly for the case of an atmospherically distorted plane wave optical signal. 


\section{CHAPTER V \\ THE OPTIMUM LOCAL OSCILLATOR WAVE FUNCTION FOR THE CASE OF AN ATMOSPHERICAILY DISTORTED OPTICAL SIGNAL}

In this Chapter the optimum fixed local oscillator wave functions are explicitly determined for the case of an atmospherically distorted plane wave optical signal and two aperture shapes. Furthermore, the corresponding maximum average signal-to-noise ratio dependence on receiver aperture size is given and is compared with that obtainable with a plane wave local oscillator wave as reported by Fried[1].

\section{A. Mutual Coherence Function}

In ordex to determine the optimum local oscillator wave function, i. e. , the solution of Eqs. (26) and (27) for the phase shape and the amplitude shape of the local oscillator beam, a knowledge of the real

and imaginary parts of the mutual coherence function, $M_{u}\left(\vec{x}, \overrightarrow{x^{\prime}}\right)$, of the received signal are required. For the case of an optical plane wave traveling through atmospheric turbulence, which is the only case to be considered here, Tatarski[ 14] presents theoretical and experimental evidence showing that the log-amplitude fluctuations have a normal distribution. Similar arguments can be made to show that the phase 
fluctuations will also have a normal distribution. Based on this fact and subject to the assumptions of isotropy and homogeneity of the statistic s of log amplitude and phase, Fried[1] demonstrates that

$$
\left\langle A(\vec{x}) A\left(\vec{x}^{\prime}\right) \sin \left(\phi(\vec{x})-\phi\left(\vec{x}^{\prime}\right)\right)\right\rangle=0
$$

and

$$
\left\langleA ( \vec { x } ) A ( \vec { x } ^ { \prime } ) \operatorname { c o s } \left(\phi(\vec{x})-\phi\left(\overrightarrow{x^{\prime}}\right)>=\vec{A}^{2} \exp \left[-\frac{1}{2} D(x)\right]\right.\right.
$$

where $\bar{A}$ is the rms value of $A(\vec{x})$ and $D(r), r=\left|\vec{x}-\overrightarrow{x^{\prime}}\right|$, is referred to as the wave structure function. It is the sum of the phase structure function

$$
D_{\phi}(r)=\left\langle\left[\phi(\vec{x})-\phi\left(\overrightarrow{x^{\prime}}\right)\right]^{2}\right\rangle
$$

and the log-amplitude structure function

$$
D_{\ell}(r)=\left\langle\left[\ell(\vec{x})-\ell\left(\overrightarrow{x^{\prime}}\right)\right]^{2}\right\rangle
$$

where

$$
\ell(\vec{x})=\ln [A(\vec{x}) / \vec{A}]
$$

Furthermore, as given by Fried[ 1],

$$
D(r)=6.88\left(r / r_{0}\right)^{5 / 3}
$$

where $r_{0}$ is a constant determined by the path, the turbulence along the path, and the optical wavelength. An alternative derivation of 
this result, based essentially on the method of Hufnagel and Stanley[ 15] is given in Appendix III. In Eqs. (92) and (93), the angle brackets denote an ensemble average. That is, in deriving Eqs. (92) and (93) the turbulent atmosphere is characterized by a family of time-independent index of refraction functions, each with an associated probability of occurrence. The amplitude and phase of the wave, as functions of position, are then determined for each family member by solving the time independent wave equation. An ensemble average then, represents a sum over all possible values of the quantity in question, each weighted by the probability of occurrence of the corresponding index of refraction function. In what follows it will be assumed that time averages over the interval $\mathrm{T}$ correspond to these ensemble averages so that, from Eqs. (23) and (92)

$$
\vec{S}\left(\vec{x}, \overrightarrow{x^{i}}\right)=0
$$

and from Eqs. (24) and (93)

$$
\vec{C}\left(\vec{x}, \overrightarrow{x^{1}}\right)=\bar{A}^{2} \exp \left[-\frac{1}{2} D(x)\right]
$$


B. Determination of Optimum

Local Oscillator Wave

Function

Having prescribed $\bar{C}$ and $\bar{S}$, the optimum local oscillator wave function may now be determined by solving the phase equation, Eq. (26), and the amplitude integral equation, Eq. (27), with these functions as input data. Thus, it follows from Eqs. (26), (98), and (99) that

$$
\theta(\vec{x})-\theta(\overrightarrow{0})=0
$$

i. e. , the optimum local oscillator phase front is flat. This is the choice made by Fried[ 1] and Gardner[2], both of whom selected a plane wave local oscillator beam, in determining the average signalto-noise ratio. It will, however, be seen below that the optimum local oscillator amplitude shape is not flat, as is the case for a plane wave, and herein will lie the difference in the results reported here as compared with that reported by Fried and Gardner.

From Eqs. (25), (98), (99), and (100) it follows that

$$
\vec{D}\left(\vec{x}, \overrightarrow{x^{\prime}}\right)=\bar{C}\left(\vec{x}, \overrightarrow{x^{\prime}}\right)=\vec{A}^{2} \exp \left[-\frac{1}{2} D(r)\right]
$$

and hence, the amplitude integral equation, Eq. (27), becomes

$$
\int W\left(\overrightarrow{x^{\prime}}\right) W(\vec{x}) \vec{A}^{2} \exp \left[-\frac{1}{2} D(x)\right] B\left(\vec{x}^{\prime}\right) \overrightarrow{d x^{\prime}}=\lambda B(\vec{x})
$$


An approximate solution of this equation, for $B(\vec{x})$, has been obtained for two cases. The first case considers a square aperture, while the second considers a circular aperture.

First then let $W(\vec{x})$ define a square aperture of area $D^{2}$. Furthermore, let this aperture be subdivided into $\mathrm{N}$ smaller squares, each of area $\Delta S$, as shown in Fig. 4. If now, the amplitude of the local oscillator beam, i. e., $B(\vec{x})$, over the square with center located at $\vec{x}=\overrightarrow{x_{i}}$

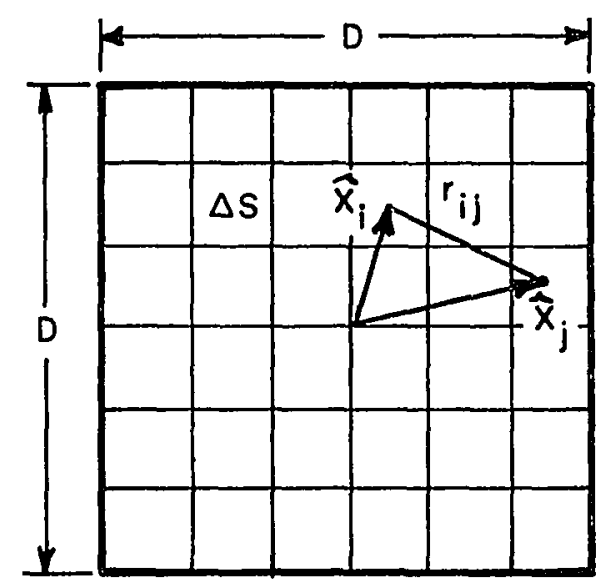

Fig. 4--Receiver aperture.

is approximated by a constant $B_{i}$, and if the integral, $\int \exp \left[-\frac{1}{2} D(r)\right] d \vec{x}^{i}$, over the square with center at $\vec{x}=\vec{x}_{i}$ is approximated by $\exp \left[-\frac{1}{2} D\left(r_{i j}\right)\right] \Delta S$ for all points in the square with center at $\vec{x}=\vec{x}_{j}$, where

$$
r_{i j}=\left|\vec{x}_{i}-\vec{x}_{j}\right|
$$


then Eq. (102) becomes

$$
\overrightarrow{A^{2}} \Delta S \sum_{i=1}^{N} B_{i} E\left(\vec{x}_{i}, \vec{x}_{j}\right)=\lambda B_{j} ; j=1,2, \ldots, N
$$

where

$$
E\left(x_{i}, x_{j}\right)=\exp \left[-\frac{1}{2} D\left(r_{i j}\right)\right]
$$

Equation (104) then, represents $N$ equations which may be solved for the $\mathrm{N}$ unknown amplitudes.

Defining the matrix

$$
E=\left(\begin{array}{ccc}
E\left(\vec{x}_{1}, \vec{x}_{1}\right) & E\left(\vec{x}_{1}, \vec{x}_{2}\right) & \cdots E\left(\vec{x}_{1}, \vec{x}_{N}\right) \\
E\left(\vec{x}_{2}, \vec{x}_{1}\right) & E\left(\vec{x}_{2}, \vec{x}_{2}\right) & \cdots E\left(\vec{x}_{2}, \vec{x}_{N}\right) \\
\vdots & \\
E\left(\vec{x}_{N}, \vec{x}_{1}\right) & E\left(\vec{x}_{N}, \vec{x}_{2}\right) & \cdots E\left(\vec{x}_{N}, \vec{x}_{N}\right)
\end{array}\right)
$$

and the column vector

$$
\underline{B}=\left|\begin{array}{c}
B_{1} \\
B_{2} \\
\vdots \\
B_{N}
\end{array}\right|
$$

These $\mathrm{N}$ equations may be written as the matrix equation

(108) $\quad \underline{E} \underline{B}=\left(\frac{\lambda}{\bar{A}^{2} \Delta S}\right) \underline{B}=\lambda^{\prime} \underline{B}$. 
Equation (108) will have a non-trivial solution for $\underline{B}$ provided

that

$$
\operatorname{det}\left(E-\frac{\lambda}{\overline{A^{2}} \Delta S}\right)=0
$$

As discussed above, the $\mathrm{N}$ roots of this equation for $\lambda$, i. e., the eigenvalues of Eq. (108), are all real and the desired amplitude solution, i. e. , the eigenvector of Eq. (108), is that corresponding to the largest eigenvalue, $\lambda_{\max }$. Furthermore, as discussed above $\lambda_{\max }$ is directly proportional to the maximum average signal-to-noise ratio. That is, from Eqs. (9) and (18), with $\lambda=\lambda_{\max }$

$$
\left(\frac{\mathrm{s}}{\mathrm{N}}\right)_{\max }=\left(\frac{\mathrm{n}}{2 \mathrm{e}}\right) \lambda_{\max }
$$

If, as shown in Appendix V, both sides of Eq. (110) are divided by $(n c)\left(\frac{r_{0}}{\sqrt{\Delta S}}\right)^{2} \bar{A}^{2} \Delta S$ the corresponding normalized signal-to-noise ratio, here denoted by $\gamma$, is comparable to the normalized signal-tonoise ratio given by Fried[ 1$]$ and denoted there by $\Psi$. Performing this division gives, for the normalized signal-to-noise ratio

$$
\gamma=\beta^{2}\left(\frac{\lambda_{\max }}{\bar{A}^{2} \Delta S}\right)=\beta^{2} \lambda_{\max }^{\prime}
$$

where

$$
\beta=\frac{\sqrt{\Delta S}}{r_{0}}
$$


and where $\lambda_{\max }^{\prime}=\frac{\lambda_{\max }}{\overline{\mathrm{A}^{2}} \Delta \mathrm{S}}$ is, from Eq. (108), the maximum eigenvalue of the $\mathrm{E}$ matrix, which might be called the mutual coherence matrix. Since the eigenvalues of this matrix depend on $r_{0}$, and since $\sqrt{N} \beta=\frac{D}{r_{0}}$ is the length of one side of the square aperture, expressed in units of $r_{0}, \gamma$ is seen to be a function of aperture size, $D$, and $r_{0}$. That is,

$$
\gamma=\gamma\left(D, r_{0}\right)=\beta^{2} \lambda^{\prime} \max
$$

C. Intensity Pattern of Optimum

Local Oscillator Beam and

Corresponding Signal-to-Noise

Ratio

Equation (108) has been solved for $\underline{B}$ and $\gamma$ for aperture sizes corresponding to $D=0.3 r_{0} ; D=0.6 r_{0} ; D=r_{0} ; D=1.5 r_{0} ;$ and $D=2 r_{0}$. The results are shown in Figs. 5 and 6.

Figure 5 ( $a$ and $b$ ) compares the normalized signal-to-noise ratio, $\gamma$, with the corresponding quantity, $\Psi$, calculated by Fried[ 1$]$. As Fried[1] points out, the important point to note is that for aperture sizes greater than $\mathrm{I}_{0}$, i. e., $\mathrm{D} / \mathrm{r}_{0}>1$, there is little significant increase in the average signal-to-noise ratio.

In Fig. $6(a, b, c, d, e)$, plots of the ratio of the optimum local oscillator beam intensity at points along the main diagonal of the aperture to the intensity at the center of the aperture, i. e. , $(B(r) / B(0))^{2}$, where $r=$ distance from center of aperture, is show: 


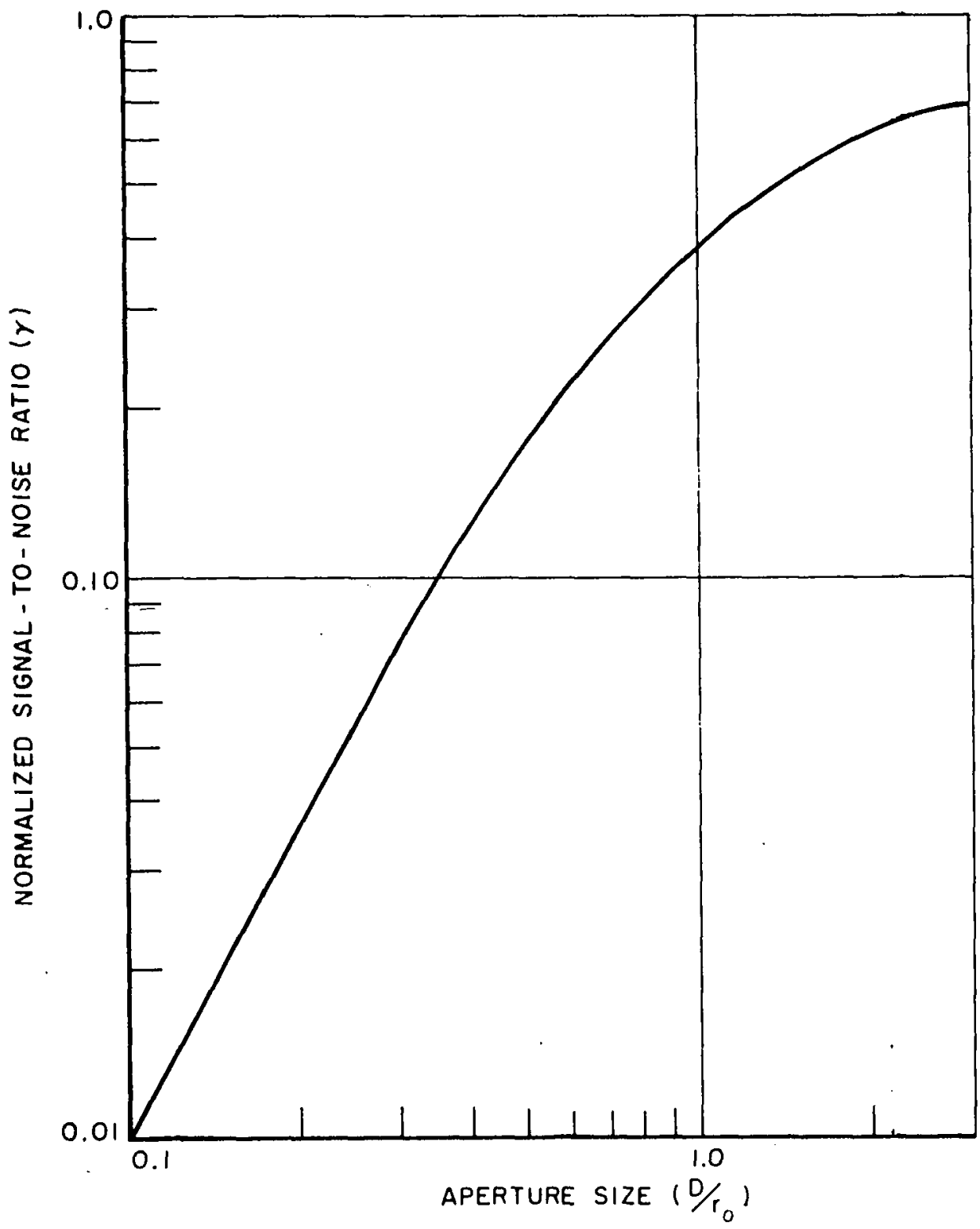

(0)

Fig. 5( a)--Normalized signal-to-noise ratio (square aperture). 


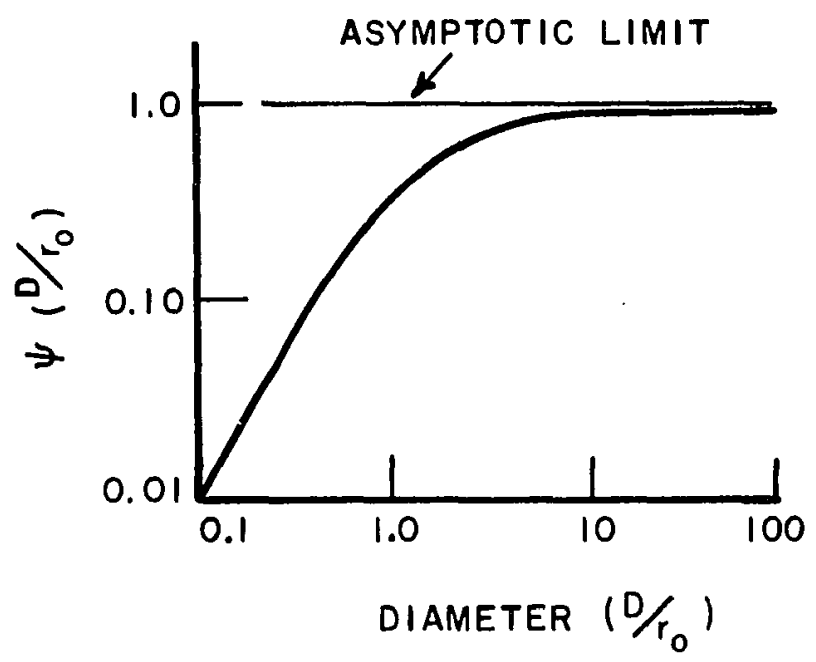

(b)

Fig. 5(b)--Fried's normalized signal-to-noise ( $r$ ound aperture).

for several aperture sizes. In all cases, the intensity pattern is symmetric about the center of the aperture. It is quite apparent from these figures that the optimum intensity pattern is not uniform across the receiver aperture. Even for an aperture size as small as $0.3 r_{0}$, the intensity falls off to 70 percent of that at the center. For an aperture size equal to $r_{0}$, it falls off to 16 percent and for an aperture size equal to $2 r_{0}$, the intensity at the corners of the aperture is only 4 percent of that at the center.

The real question of interest here is whether or not the optimum local oscillator wave function leads to a significant increase in the average signal-to-noise ratio over that obtained for the case 


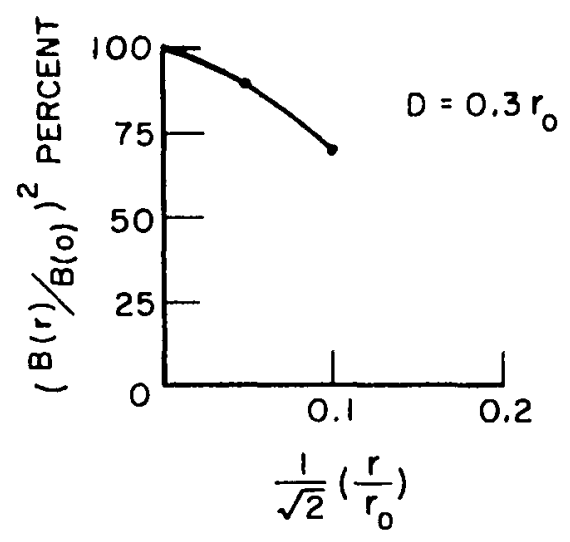

(a)

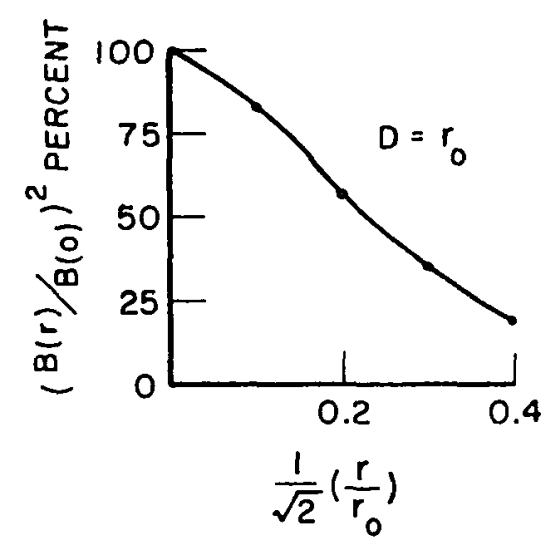

(c)

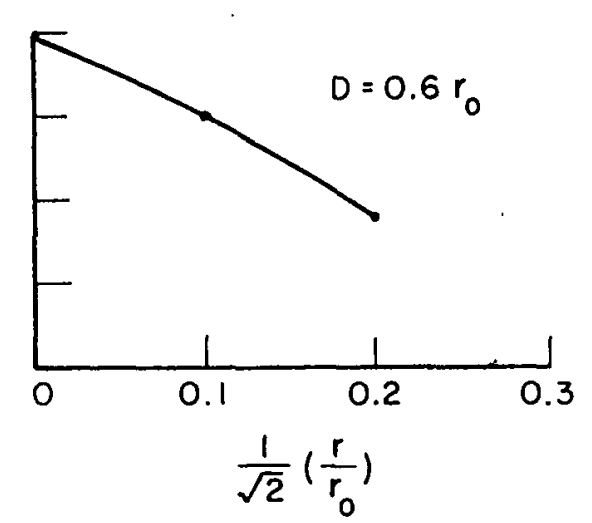

(b)

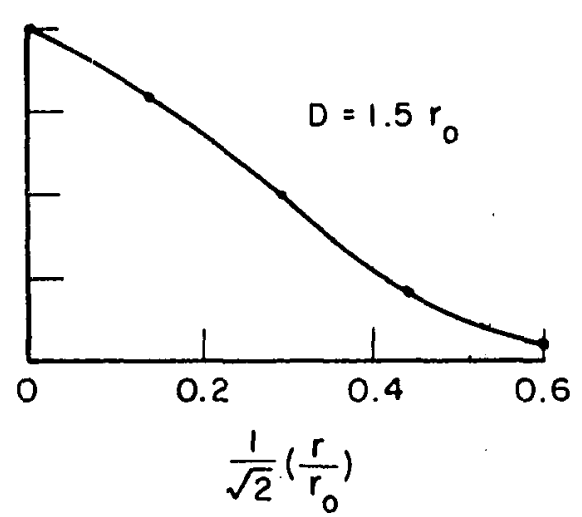

(d)

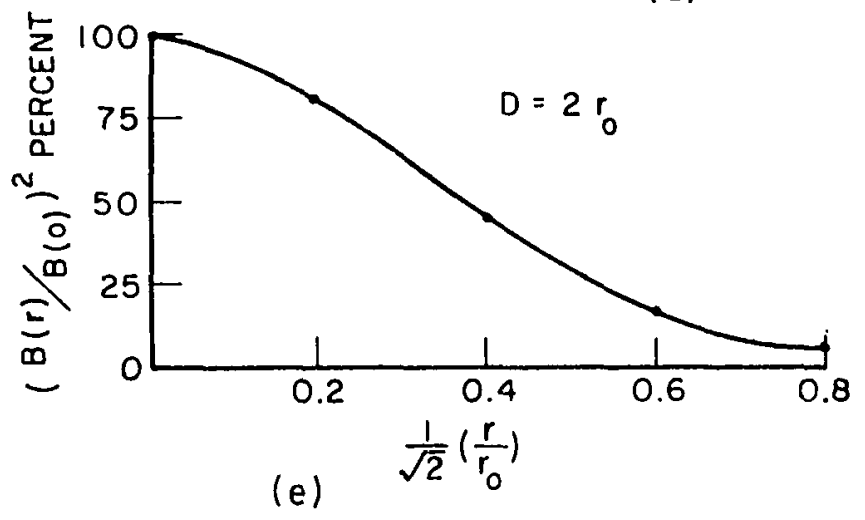

Fig. 6--Local oscillator intensity patterns (square aperture). 
of a plane wave local oscillator as reported by Fried[1]. In order to determine this, a plot of

$$
G=\frac{(S / N) \max }{(S / N) \text { Plane Wave }}
$$

is shown in Fig. 7. An expression for G may be obtained as follows: For the case of a plane wave local oscillator the average signal power is, from Eq. (7) with $M_{u}\left(\vec{x}, \overrightarrow{x^{\prime}}\right)=\bar{A}^{2} \exp \left[-\frac{1}{2} D(r)\right]$ and $V(\vec{x}) V^{* k}\left(\overrightarrow{x^{\prime}}\right)=$ $B(\vec{x}) B\left(\overrightarrow{x^{\prime}}\right)=B_{O}^{2}$,

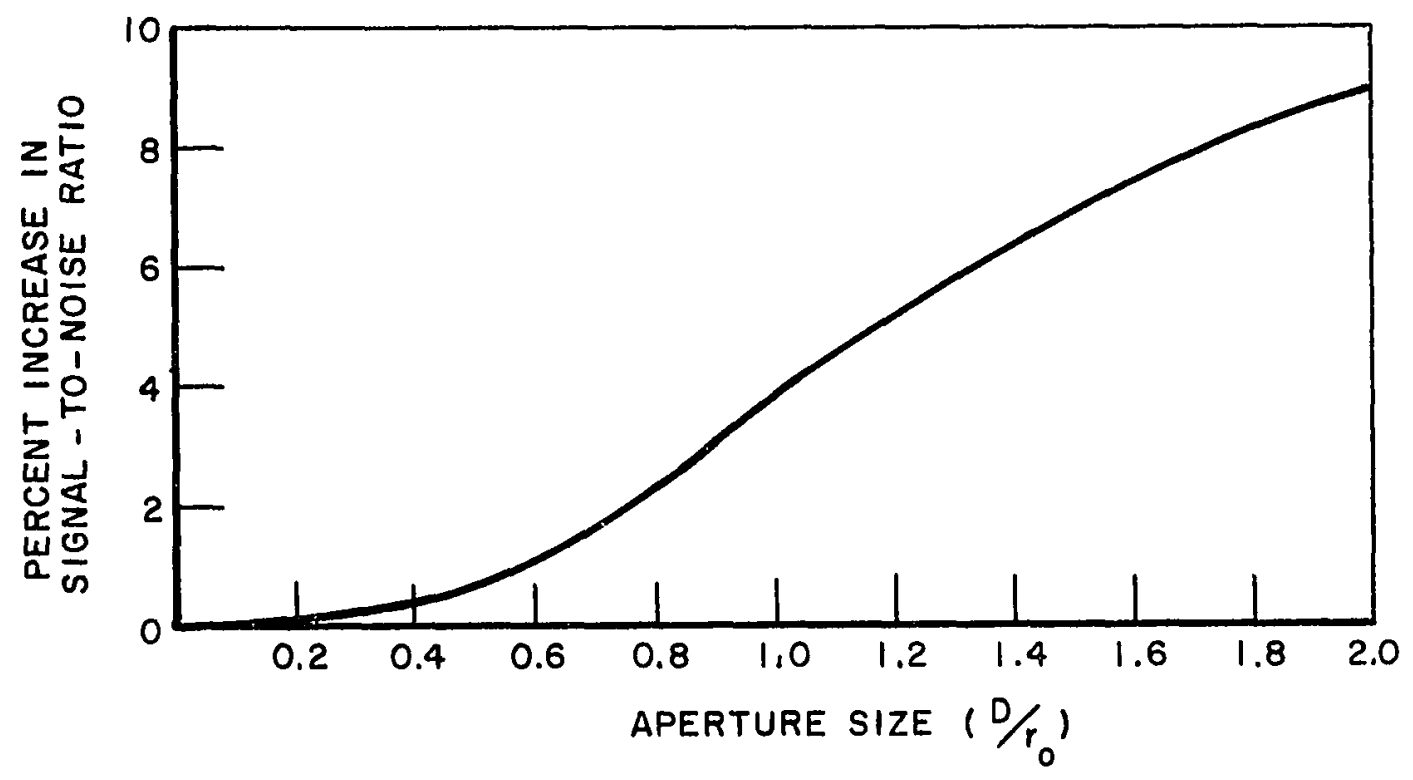

Fig. 7--Percent increase in $(S / N)-$ square aperture. 


$$
P_{0}^{\prime}=\frac{1}{2} \eta^{2} B_{O}^{2} \vec{A}^{2} \iint W(\vec{x}) W\left(\overrightarrow{x^{\prime}}\right) \exp \left[-\frac{1}{2} D(r)\right] d \vec{x} d \overrightarrow{x^{\prime}}
$$

Making the same approximations as in deriving Eq. (104), this becomes

$$
P_{0}^{\prime}=\frac{1}{2} \eta^{2} B_{O}^{2} \vec{A}^{2}(\Delta S)^{2} \sum_{i, j=1}^{N} E\left(\vec{x}_{i}, \vec{x}_{j}\right)
$$

The corresponding local oscillator power is, from Eq. (8) with $B(\vec{x})=0$ outside the aperture, given by

$$
\begin{aligned}
P_{L}^{\prime} & =\frac{1}{2} \eta \int W(\vec{x}) B_{0}^{2} \overrightarrow{d x} \\
& =\frac{1}{2} \eta B_{O}^{2} N \Delta S
\end{aligned}
$$

The average signal-to-noise ratio is then, for the plane wave case,

$$
\begin{aligned}
(S / N)_{\text {P. } W .} & =\frac{1}{2 e} \frac{P_{0}^{\prime}}{P_{L}^{\prime}} \\
& =\frac{1}{2 e} \eta \vec{A}^{2} \Delta S \frac{1}{N} \sum_{i, j=1}^{N} E\left(\vec{x}_{i}, \vec{x}_{j}\right)
\end{aligned}
$$

Finally, from Eqs. (114), (110) and (118)

$$
G=\frac{\lambda_{\max }}{\bar{A}^{2} \Delta S} \frac{N}{\sum_{i, j=1}^{N} E\left(\vec{x}_{i}, \vec{x}_{j}\right)}
$$

or 
(120)

$$
G=\lambda_{\max }^{\prime} \frac{N}{\sum_{i, j=1}^{N} E\left(\vec{x}_{i}, \vec{x}_{j}\right)}
$$

It is evident from Fig. (7) that there is indeed practically no significant increase in the average signal-to-noise ratio, over that for the plane wave case. In fact, for an aperture size equal to $r_{0}$, the increase is only 4 percent and even for an aperture size of $2 \mathrm{r}_{\mathrm{O}}$, it is only 9 percent. It appears then that very little is to be gained, insofar as average signal-to-noise ratio is concerned, by shaping the local oscillator beam according to the method developed here.

Consider next the case of a circular aperture. It should be noted here that the solution of this problem, which makes use of the circular symmetry of the optimum local oscillator beams amplitude pattern, $B_{o}(\vec{x})$, offers greater accuracy without an increase the number of unknowns, $B_{0}\left(\vec{x}_{j}\right) ; i=1,2, \ldots, N[17]$.

Thus, for a circular aperture of radius $(D / 2)$, Eq. (102) becomes, in terms of polar coordinates $r$ and $\theta$,

$$
\begin{gathered}
\overrightarrow{A^{2}} \int_{0}^{D / 2} r^{\prime} d r^{\prime} \int_{0}^{2 \pi} d \theta^{\prime} B_{0}\left(r^{\prime}\right) \exp \left[-\frac{1}{2} D\left(r^{2}+r^{\prime 2}-2 r r^{\prime} \cos \left(\theta^{\prime}-\theta\right)\right)\right] \\
=\lambda B_{o}(r) ; 0 \leq r \leq D / 2 .
\end{gathered}
$$


Since the $\theta^{\prime}$ integration is independent of $B_{0}$, Eq. (12l) may be written in the form

$$
\bar{A}^{2} \int_{0}^{D / 2} B_{0}\left(r^{\prime}\right) Q\left(r, r^{\prime}\right) r^{\prime} d r^{\prime}=\lambda B_{0}(r)
$$

where

$$
Q\left(r, r^{\prime}\right)=\int_{0}^{2 \pi} \exp \left[-\frac{1}{2} D\left(r^{2}+r^{\prime 2}-2 r r^{\prime} \cos \left(\theta^{\prime}-\theta\right)\right)\right] d \theta^{\prime}
$$

and is independent of $\theta$.

Lettir.g $U=\frac{r}{(D / 2)}$, Eq. (122) becomes

$$
\begin{gathered}
\int_{0}^{1} B_{0}\left(U^{\prime}\right) Q\left(\frac{D}{2} U, \frac{D}{2} U^{\prime}\right) U^{\prime} d U^{\prime}=\left(\frac{\lambda}{A^{2}\left(\frac{D}{2}\right)^{2}}\right) B_{0}(U) ; \\
0 \leq U \leq 1 .
\end{gathered}
$$

With the function $Q$ evaluated by numerical integration, the one dimensional integral equation, Eq. (124), may, in precisely the same manner as for the square aperture, be reduced to a set of $S$ algebraic equations in $S$ unknown amplitudes, $B_{0}=B_{0}(0) ; B_{1}=B_{0}\left(\frac{1}{S}\right) ; \ldots$; $\mathrm{B}_{\mathrm{S}-1}=\mathrm{B}_{\mathrm{O}}\left(\frac{\mathrm{S}-1}{\mathrm{~S}}\right)$. These $\mathrm{S}$ equations, expressed in matrix form, are

$$
\left(K_{i j}\right)\left(B_{j}\right)=\lambda^{\prime}\left(B_{i}\right) ; i, j=0,1, \cdots, s-1
$$

where 


$$
K_{i j}=j \int_{0}^{2 \pi} \exp \left\{-3.44\left(\frac{D}{2 r_{O} S}\right)^{5 / 3}\left[i^{2}+j^{2}-2 i j \cos \theta^{\prime}\right]^{5 / 6}\right\} d \theta^{\prime}
$$

and

$$
\lambda^{\prime}=\frac{\lambda}{\bar{A}^{2}\left(\frac{D}{2 S}\right)^{2}}
$$

The maximum eigenvalue, $\lambda^{\prime}=\lambda_{\max }^{\prime}$, and the corresponding eigenvector, $\left(B_{i}\right)$ opt. , have been determined for several values of aperture diameter $D$. The corresponding maximum, normalized average signal-to-noise ratio, $\gamma$, defined in the same manner as for the square aperture is

$$
y=\frac{4}{\pi}\left(\frac{D}{2 S}\right)^{2} \lambda_{\max }^{\prime}
$$

The results of these calculations are given in Figs. 8 and 9. The results agree quite closely with those for the square aperture.

It should be noted here that the amplitude shape of the optimum local oscillator beam depends critically on the size of the aperture. That is, for example, the amplitude pattern for the aperture of diameter $2 r_{o}$ is not obtained by simply "extending" the pattern obtained for the aperture of diameter $r_{0}$. Indeed, at a distance of $r_{0} / 2$ from the center of the $2 r_{0}$ aperture (which corresponds to the edge of the $r_{0}$ aperture) the intensity is 62 percent of that at the center. On the other hand, at the edge of the $r_{0}$ aperture the intensity is only 30 percent of that of the center. 


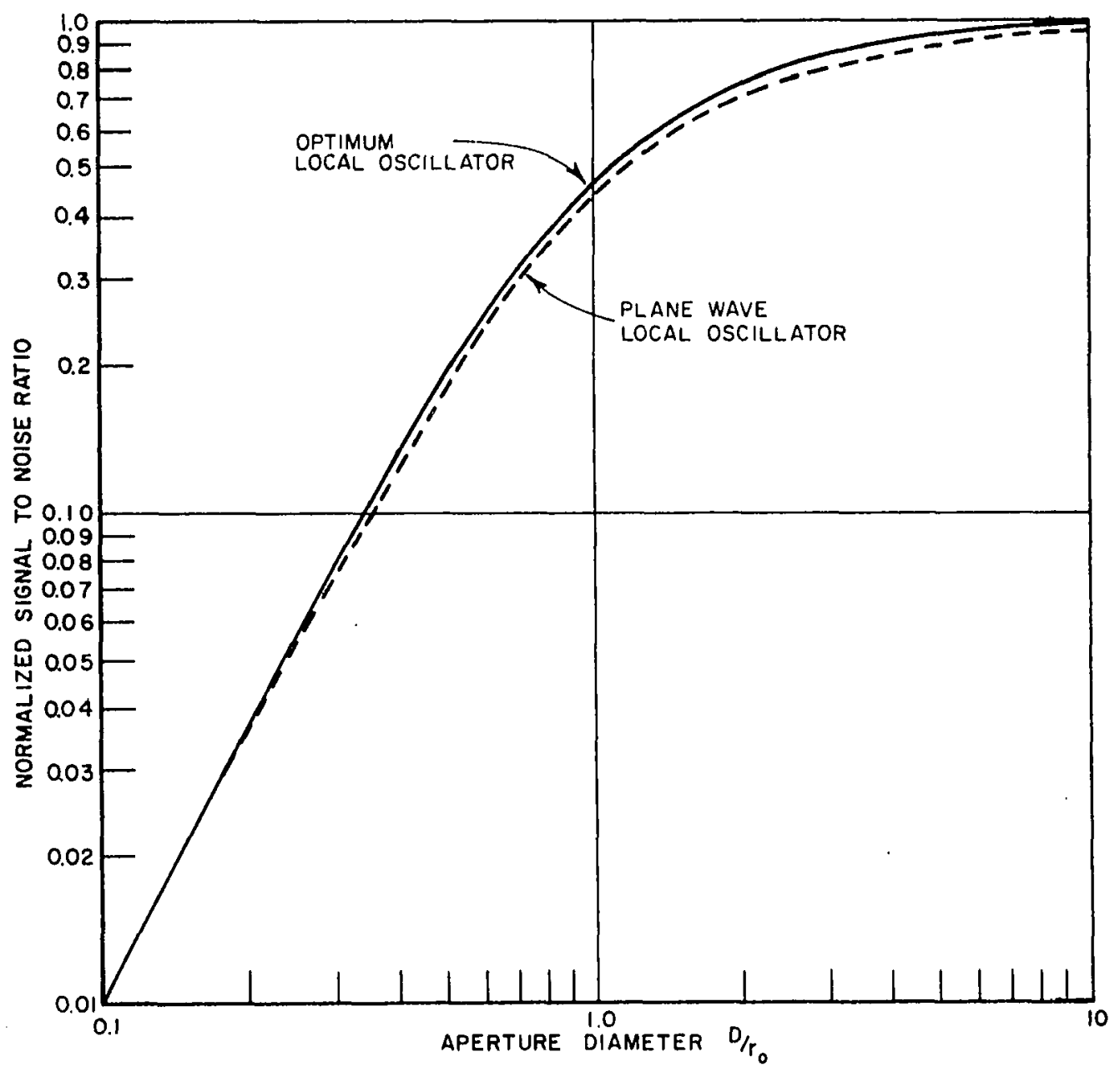

Fig. 8--Normalized signal-to-noise ratio (round aperture). 


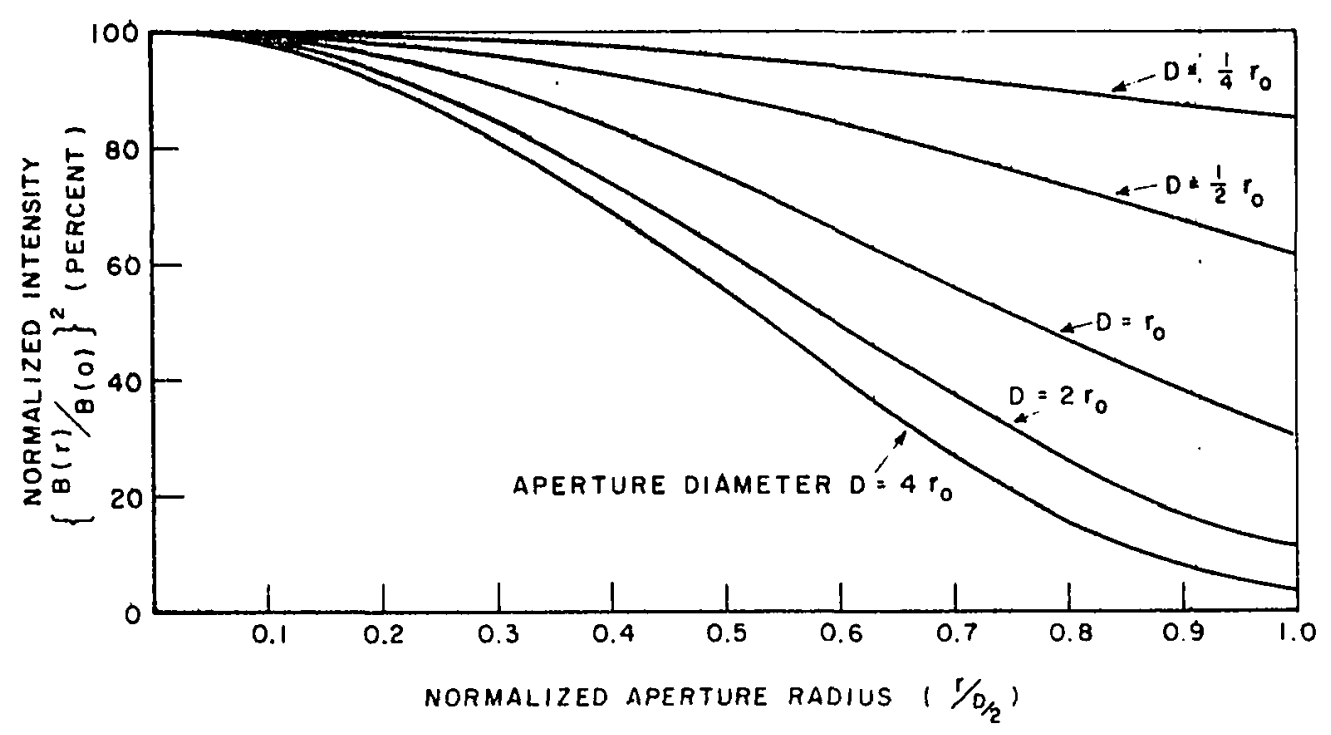

Fig. 9--Normalized intensity patterns (round aperture).

In summary then, the local oscillator shapes and signal-tonoise ratios have been derived for the important case of an atmospherically degraded plane wave. It has been shown that the optimum local oscillator beam does not lead to a significant increase, compared with the plane wave local oscillator beam, in the average signal-to-noise ratio. This is so, even though its shape differs markedly from that of the plane wave. 


\section{CHAPTER VI \\ EQUIVALENCE OF DIFFERENT HETERODYNE \\ DETECTION SYSTEMS AND VARIANCE \\ OF BEAT SIGNAL POWER}

In the previous Chapter it was shown, for the case of an atmospherically distorted plane wave, that no significant improvement in the average signal-to-noise ratio, over that for the case of a plane wave local oscillator, can be obtained by judiciously choosing the shape of the local oscillator wave over the receiver aperture. It is, however, conceivable that an additional increase might be obtainable by pre-processing the received signal in some manner before mixing it with a local oscillator beam.

It is, however, shown in this Chapter that (at least for a general class of time invariant, linear pre-processing operations) no additional increase in the average signal-to-noise ratio can be obtained. This statement is true, no matter what the nature of the distortion of the received optical signal - atmospheric, or otherwise. 


\section{A. Discussion of Different \\ Detection Methods}

The heterodyne detection configuration under consideration is shown in Fig. 10. The spatial filters may adjust the phase and/or amplitude of the signals incident on them, as indicated. The effect of the linear operator is to generate the $\operatorname{signal} z(\vec{y}, t)$ from the received signal $U(\vec{x}, t) W(\vec{x}) G_{1}(\vec{x})$. This device may, for example, be a lens and in this case it can be shown[16] that $Z(\vec{y}, t)$ is proportional to the spatial Fourier transform of $U(\vec{x}, t) W(\vec{x}) G_{1}(\vec{x})$. This is, of course, not the only possible lens operation. In any case, any time invariant linear relationship between $\mathrm{Z}$ and $\mathrm{UWG}_{1}$ can be expressed in the form

$$
Z(\vec{y}, t)=\int\left\{U(\vec{x}, t) W(\vec{x}) G_{1}(\vec{x})\right\} L(\vec{x}, \vec{y}) d \vec{x}
$$

For example, in the case where a lens is used to produce the spatial Fourier transform of $U(\vec{x}, t) W(\vec{x}), L(\vec{x}, \vec{y})=($ const. $) e^{-\overrightarrow{j x} \cdot \vec{y}}$. The one restriction to be placed on the linear operator is that it be such that the power in the signal $Z(\vec{y}, t)$ equal the power in the signal $U(\vec{x}, t) W(\vec{x}) G_{1}(\vec{x})$. Stated simply, this means that the linear operator removes no light from the beam it receives, but merely rearranges the incident light to form a new signal, as is the case, for example, with the large lens. The only points at which energy may be removed from the beam are at the spatial filters No. 1 and No. 2. In the case 


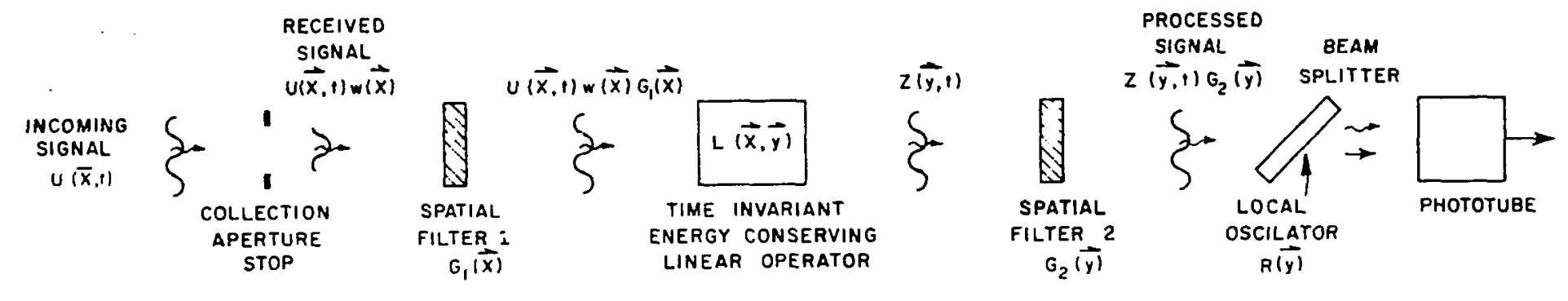

Fig. 10--Time invariant detection arrangement. 
of the lens, performing a Fourier transform, for example, the spatial filter No. 2 could attenuate and/or adjust the phase of the different spatial frequency components (i. e., $Z\left(\vec{y}_{i}, t\right)$ ) of the $\operatorname{signal} U(\vec{x}, t) W(\vec{x})$ $G_{1}(\vec{x})$.

The condition to be imposed on $L(\vec{x}, \vec{y})$ by the requirement that no energy be removed from the incident beam (here denoted by $V(\vec{x}, t)$ ) is determined by the equation

$$
\int z(\vec{y}, t) z^{*}(\vec{y}, t) d \vec{y}=\int V(\vec{x}, t) V^{*}(\vec{x}, t) d \vec{x}
$$

where

$$
Z(\vec{y}, t)=\int V(\vec{x}, t) L(\vec{x}, \vec{y}) d \vec{x}
$$

Subsituting Z from Eq. (131) into Eq. (130) gives

$$
\begin{gathered}
\int_{L^{2} \vec{y} \int_{d} d \vec{x}} V(\vec{x}, t) L(\vec{x}, \vec{y}) \int d \vec{x} V^{*}(\vec{x}, t) L^{*}(\vec{x}, \vec{y}) \\
\quad=\int V(\vec{x}, t) V^{*}(\vec{x}, t) d \vec{x}
\end{gathered}
$$

or

$$
\begin{gathered}
\int d \vec{x} \int d \vec{x}^{\prime} V(\vec{x}, t) V^{*}\left(\overrightarrow{x^{\prime}}, t\right) \int L(\vec{x}, \vec{y}) L^{*}\left(\overrightarrow{x^{\prime}}, \vec{y}\right) d \vec{y} \\
=\int V(\vec{x}, t) V^{*}\left(\overrightarrow{x^{\prime}}, t\right) d \vec{x}
\end{gathered}
$$


Defining the function

$$
I\left(\vec{x}, \vec{x}^{\prime}\right)=\int L(\vec{x}, \vec{y}) L^{*}\left(\vec{x}^{\prime}, \vec{y}\right) d \vec{y}
$$

Eq. (132) becomes

$$
\begin{gathered}
\int d \vec{x} \int d \overrightarrow{x^{\prime}} V(\vec{x}, t) V^{*}\left(\overrightarrow{x^{\prime}}, t\right) I\left(\vec{x}, \overrightarrow{x^{\prime}}\right) \\
\quad=\int V(\vec{x}, t) V^{*}(\vec{x}, t) d \vec{x}
\end{gathered}
$$

It is shown in Appendix IV that if Eq. ( 134$)$ is to hold for all $V(\vec{x}, t)$, then

$$
\begin{aligned}
I\left(\vec{x}, \overrightarrow{x^{i}}\right) & =\int L(\vec{x}, \vec{y}) L^{*}\left(\overrightarrow{x^{\prime}}, \vec{y}\right) d \vec{y} \\
& =\delta\left(\vec{x}-\overrightarrow{x^{\prime}}\right)
\end{aligned}
$$

where $\delta$ is the Dirac delta function.

\section{B. Equivalence of Different Methods}

In order to show that no additional improvement in the average signal-to-noise ratio can be obtained by performing any such preprocessing operation on the received signal, two steps are sufficient. The first step is to determine the maximum average signal-to-noise ratio that can be obtained from the processed signal $Z(\vec{y}, t) G_{2}(\vec{y})$ and the second is to show that it is equal to the maximum average signalto-noise ratio that can be obtained from the received signal $U(\vec{x}, t) W(\vec{x})$. 
Consider first the signal $\mathrm{Z}(\overrightarrow{\mathrm{y}}, \mathrm{t}) \mathrm{G}_{2}(\overrightarrow{\mathrm{y}})$. The average signal-tonoise ratio is, from Eqs. (9), (7), and (8)

$$
\begin{aligned}
& \frac{S}{N}=\frac{1}{2 e} \frac{P_{O}}{P_{L}} \\
& =\frac{1}{2 e} \frac{\left.\frac{1}{2} \eta^{2} \iint R^{*}(\vec{y}) R\left(\overrightarrow{y^{\prime}}\right) G_{2}(\vec{y}) G_{2}^{*}\left(\overrightarrow{y^{\prime}}\right)<Z(\vec{y}, t) z^{*}\left(\vec{y}^{\prime}, t\right)\right\rangle d \vec{y} d \overrightarrow{y^{\prime}}}{\frac{1}{2} \eta \int R(\vec{y}) R^{*}(\vec{y}) d \vec{y}} .
\end{aligned}
$$

Note that $G_{2}(\vec{y})$ appears only in the numerator of $S / N$. It follows that for all values of $\vec{y}$ for which $R(\vec{y}) \neq 0$, the magnitude of $G_{2}(\vec{y})$ should be set at its maximum possible value, namely one. For those values of $\vec{y}$ for which $R(\vec{y})=0,\left|G_{2}(\vec{y})\right|$ may be set equal to one. Now note further that an examination of $\mathrm{S} / \mathrm{N}$ shows that the phase of either $R(\vec{y})$ or $G(\vec{y})$ may be adjusted to maximize the numerator of $S / N$ with no effect on the denominator of $S / N$. It follows that the phase of $G_{2}(\vec{y})$ may be set equal to zero. Then $G_{2}(\vec{y})$ will be set equal to one and thus, $G_{2}(\vec{y})$ has afforded no improvement.

The analysis to follow should be considered to apply to the signals $U_{W} G_{1}$ and $Z$. For notational simplicity, however, the factor $G_{1}(\vec{x})$ will be absorbed into the factor $U(\vec{x}, t)$. Thus, let $U W G_{1}=U^{\prime} W$. The result of the following analysis will be that the signals $U$ ' $W$ and $Z$ lead to the same maximum value of $S / N$. 
Note, then, that the maximum average signal-to-noise ratio that can be obtained from the signal $\mathrm{Z}(\vec{y}, t)$ is achieved by choosing the local oscillator wave function, denoted $R(\vec{y})$, to be that solution of the integral equation,

$$
\int M_{Z}\left(\vec{y}, \overrightarrow{y^{\prime}}\right) R\left(\overrightarrow{y^{\prime}}\right)=\lambda R(\vec{y})
$$

where

$$
M_{z}\left(\vec{y}, \overrightarrow{y^{\prime}}\right)=\left\langle z(\vec{y}, t) z^{*}\left(\vec{y}^{\prime}, t\right)\right\rangle
$$

is the mutual coherence function of the signal $z(\vec{y}, t)$. This follows directly from the derivation of Eq. (16) by replacing $U(\vec{x}, t) W(\vec{x})$ by $Z(\vec{y}, t)$ and $V(\vec{x})$ by $R(\vec{y})$. Furthermore, with reference to the discussion following Eq. (16), it follows that the maximum average signal-to-noise ratio that can be obtained from the signal $\mathrm{Z}(\overrightarrow{\mathrm{y}}, \mathrm{t})$ is directly proportional to the maximum eigenvalue, $\lambda_{\max }$, of Eq. (136). Thus, in order to show that the signals $U^{\prime} W$ and $Z$ lead to the same maximum value of $\mathrm{S} / \mathrm{N}$ it is sufficient to show that the eigenvalues of Eq. (16)

$$
\int M_{U^{\prime}}\left(\vec{x}, \overrightarrow{x^{\prime}}\right) V\left(\overrightarrow{x^{\prime}}\right) d \overrightarrow{x^{\prime}}=\lambda_{U^{\prime}} V(\vec{x})
$$

are identical with the eigenvalues of Eq. (136)

$$
\int M_{Z}\left(\vec{y}, \overrightarrow{y^{\prime}}\right) R\left(\overrightarrow{y^{\prime}}\right) d \overrightarrow{y^{\prime}}=\lambda_{Z} R(\vec{y})
$$


To show this, it is sufficient to show that every eigenvalue of Eq. (16) is an eigenvalue of Eq. (136) and vice-versa. That such is the case obviously depends upon the relationship between $M_{Z}$ and $M_{U^{\prime}}$. This relationship is, from Eqs. (137) and (129)

$$
M_{Z}\left(\vec{y}, \overrightarrow{y^{\prime}}\right)=\int d \vec{x} \int d \overrightarrow{x^{\prime}} L(\vec{x}, \vec{y}) M_{U^{\prime}}\left(\vec{x}, \vec{x}^{\prime}\right) L^{*}\left(\vec{x}^{\prime}, \vec{y}^{\prime}\right)
$$

and hence, Eq. (136) may be written

$$
\begin{aligned}
& \int d \overrightarrow{y^{\prime}} \int d \vec{x} \int d \overrightarrow{x^{\prime}} L(\vec{x}, \vec{y}) M_{U^{\prime}}\left(\vec{x}, \overrightarrow{x^{\prime}}\right) L^{*}\left(\vec{x}^{\prime}, \vec{y}^{\prime}\right) R\left(\vec{y}^{\prime}\right) \\
& =\lambda_{Z} R(\vec{y}) \text {. }
\end{aligned}
$$

Then, multiplying both sides of Eq. (16) by $L(\vec{x}, \vec{y})$ and integrating with respect to $\vec{x}$ gives

$$
\begin{aligned}
\int \mathrm{d} \vec{x} \int & d \overrightarrow{x^{\prime}} L(\vec{x}, \vec{y}) M_{U^{\prime}}\left(\vec{x}, \overrightarrow{x^{\prime}}\right) V\left(\overrightarrow{x^{\prime}}\right) \\
& =\lambda_{U^{\prime}} \int V(\vec{x}) L(\vec{x}, \vec{y}) d \vec{x}
\end{aligned}
$$

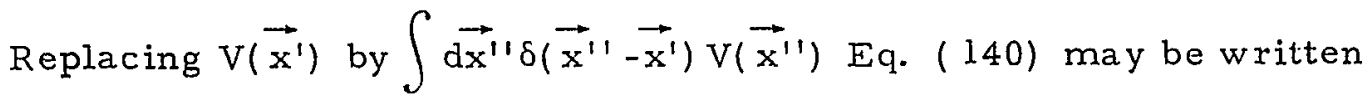

$$
\begin{aligned}
& \int \overrightarrow{d x} \int \overrightarrow{d x^{\prime} J}(\vec{x}, \vec{y}) M_{U^{\prime}}\left(\vec{x}, \overrightarrow{x^{\prime}}\right) \int d \overrightarrow{x^{\prime \prime}} \delta\left(\overrightarrow{x^{\prime \prime}}-\overrightarrow{x^{\prime}}\right) V\left(\overrightarrow{x^{\prime \prime}}\right) \\
& =\lambda_{U^{\prime}} \int V(\vec{x}) L(\vec{x}, \vec{y}) d \vec{x}
\end{aligned}
$$

From Eq. $(135), \delta\left(\vec{x}^{\prime \prime}-\vec{x}^{\prime}\right)$ may be written in the form 


$$
\delta\left(\vec{x}^{\prime \prime}-\vec{x}^{\prime}\right)=\int L^{*}\left(\overrightarrow{x^{\prime}}, \overrightarrow{y^{\prime}}\right) L\left(\overrightarrow{x^{\prime \prime}}, \overrightarrow{y^{\prime}}\right) \overrightarrow{d y^{\prime}}
$$

and hence Eq. (141) may be written

$$
\begin{gathered}
\int d \vec{x} \int d \overrightarrow{x^{\prime}} L(\vec{x}, \vec{y}) M_{U^{\prime}}\left(\vec{x}, \vec{x}^{\prime}\right) \int d \vec{x}^{\prime \prime} \int d \vec{y}^{\prime} L^{*}\left(\overrightarrow{x^{\prime}}, \overrightarrow{y^{\prime}}\right) \\
L\left(\overrightarrow{x^{\prime \prime}}, \overrightarrow{y^{\prime}}\right) V\left(\vec{x}^{\prime \prime}\right)=\lambda U^{\prime} \int V(\vec{x}) L(\vec{x}, \vec{y}) d \vec{x}
\end{gathered}
$$

Finally, by interchanging order of integration, Eq. (143) may be written

$$
\begin{aligned}
& \int d \overrightarrow{y^{\prime}} \int d \vec{x} \int d \vec{x}^{\prime} L(\vec{x}, \vec{y}) M_{U^{\prime}}\left(\vec{x}, \overrightarrow{x^{\prime}}\right) L^{*}\left(\overrightarrow{x^{\prime}}, \overrightarrow{y^{\prime}}\right) \\
& \left\{\int \overrightarrow{d x^{\prime \prime}} V\left(\overrightarrow{x^{\prime \prime}}\right) L\left(\overrightarrow{x^{\prime \prime}}, \overrightarrow{y^{\prime}}\right)\right\}=\lambda_{U^{\prime}} \int \overrightarrow{d x} V(\vec{x}) L(\vec{x}, \vec{y})
\end{aligned}
$$

Comparing Eqs. (144) and (139), it follows that

$$
R(\vec{y})=\int \overrightarrow{d x} V(\vec{x}) L(\vec{x}, \vec{y})
$$

is a solution of Eq. (136) corresponding to $\lambda_{Z}=\lambda_{i}$ provided that $V(\vec{x})$ is a solution of Eq. (16) corresponding to $\lambda_{U^{\prime}}=\lambda_{i}$. Thus, every eigenvalue of Eq. (16) is an eigenvalue of Eq. (136). In order to show the converse of this statement, i. e., that every eigenvalue of Eq. (136) is an eigenvaiue of Eq. (16), multiply both sides of Eq. (139) by $L^{*}\left(\overrightarrow{x^{\prime \prime}}, \vec{y}\right)$ and integrate with respect to $\vec{y}$ to give 
( 146$)$

$$
\begin{aligned}
\int d \vec{x} \int d \vec{x}^{\prime} & \int d \vec{y} L(\vec{x}, \vec{y}) L^{*}\left(\overrightarrow{x^{\prime \prime}}, \vec{y}\right) M_{U^{\prime}}\left(\vec{x}, \overrightarrow{x^{\prime}}\right) \\
& \int d \vec{y}^{\prime} L^{*}\left(\overrightarrow{x^{\prime}}, \vec{y}^{\prime}\right) R\left(\vec{y}^{\prime}\right)=\lambda_{Z} \int \overrightarrow{d y} R(\vec{y}) L\left(\overrightarrow{x^{\prime \prime}, \vec{y}}\right) .
\end{aligned}
$$

From Eq. (135), the $\vec{y}$ integration on the left hand side of Eq. (146) gives rise to $\delta\left(\vec{x}-\overrightarrow{x^{\prime \prime}}\right)$. Then, performing the $\vec{x}$ integration, Eq. (146) becomes

$$
\begin{aligned}
& \int d \vec{x}^{\prime} M_{U^{\prime}}\left(\overrightarrow{x^{\prime \prime}}, \overrightarrow{x^{\prime}}\right)\left\{d \vec{y}^{\prime} L^{*}\left(\overrightarrow{x^{\prime}}, \overrightarrow{y^{\prime}}\right) R\left(\vec{y}^{\prime}\right)\right\} \\
& =\lambda_{Z} \int \mathrm{d} \overrightarrow{y I}^{*}\left(\vec{x}^{\prime \prime}, \vec{y}\right) R(\vec{y})
\end{aligned}
$$

Finally, comparing Eqs. (147) and (16), it follows that

$$
V(\vec{x})=\int L^{*}(\vec{x}, \vec{y}) R(\vec{y}) \overrightarrow{d y}
$$

is a solution of Eq. (16) corresponding to $\lambda_{U^{\prime}}=\lambda_{j}$ provided that $R(\vec{y})$ is a solution of Eq. (136) corresponding to $\lambda_{Z}=\lambda_{j}$. Thus, every eigenvalue of Eq. (136) is an eigenvalue of Eq. (16). Furthermore, the relationship between the corresponding eigenvectors, as given by Eq. (145), is precisely the same as that between the signals $U^{\prime}(\vec{x}, t) W(\vec{x})$ and $z(\vec{y}, t)$. It follows then, that

$$
\lambda_{Z \max }=\lambda_{U^{\prime} \max }
$$


which, as discussed above, implies that the maximum average signalto-noise ratio that can be obtained from the $\operatorname{signal} \mathrm{Z}(\overrightarrow{\mathrm{y}}, \mathrm{t})$ is equal to the maximum average signal-to-noise ratio that can be obtained from the signal $U^{\prime}(\vec{x}, t) W(\vec{x})$.

It is a simple matter now to show that the filter function $G_{1}(\vec{x})$ may be set equal to one. The argument is precisely that used to show that $G_{1}(\vec{y})$ may be set equal to one.

It has been shown then, that there is no possibility of obtaining a larger average signal-to-noise by employing an optical system of the form discusised.

\section{Variance of Beat Signal Power}

In this section the variance of the beat signal power (i. e. , fluctuations about the average value) is discussed. As mentioned in the introduction, the variance may actually be more important than the average signal-to-noise ratio in determining the performance of a communications link. A simple extension of the above theory will, however, show that not only is the maximum average signal-to - noise ratio independent of the choice of the optical system, but so too are the corresponding instantaneous values, and hence, the variance of the beat signals. To show this, let the optimum local oscillator wave function for heterodyning with the received signal $U(\vec{x}, t) W(\vec{x})$ be denoted by $V_{o}(\vec{x})$. Then, from Eq. (145), the optimum local 
oscillator wave function for heterodyning with the $\operatorname{signal} z(\vec{y}, t)$, obtained by passing the signal $U(\vec{x}, t) W(\vec{x})$ through the optical system, is given by

$$
R_{0}(\vec{y})=\int d \vec{x} V_{0}(\vec{x}) L(\vec{x}, \vec{y})
$$

That is, $R_{0}(\vec{y})$ is obtained by passing $V_{o}(\vec{x})$ through the same optical system as was $U(\vec{x}, t) W(\vec{x})$. The objective now will be to show that $R_{o}(\vec{y})$ and $z(\vec{y}, t)$ produce the same beat signal current (vs. t) as does $V_{0}(\vec{x})$ and $U(\vec{x}, t) W(\vec{x})$. Thus, from Eq. (5) the beat signal current for $V_{0}(\vec{x})$ and $U(\vec{x}, t) W(\vec{x})$ is given by

$$
I_{U}=\operatorname{Re} e^{j \omega t} \eta \int W(\vec{x}) V_{o}^{*}(\vec{x}) U(\vec{x}, t) d \vec{x}
$$

Similarly, the beat signal current for the pair $R_{0}(\vec{y})$ and $z(\vec{y}, t)$ is given by

$$
I_{Z}=\operatorname{Re} e^{j \omega t} \eta \int R_{0}^{* *}(\vec{y}) Z(\vec{y}, t) d \vec{y} .
$$

Substituting for $R_{0}(\vec{y})$ and $z(\vec{y}, t)$ their equivalents, as given by Eqs. (150) and (129) respectively, ane obtains

$$
\begin{aligned}
I_{Z}=\operatorname{Re} e^{j \omega t} \eta \int d \vec{y} \int d \vec{x} v_{o}^{* *}(\vec{x}) L^{* ;}(\vec{x}, \vec{y}) \\
\qquad d \vec{x} W(\vec{x}) U(\vec{x}, t) L(\vec{x}, \vec{y})
\end{aligned}
$$

or 


$$
\begin{array}{r}
I_{Z}=\operatorname{Re} e^{j \omega t} \eta \iiint W\left(\overrightarrow{x^{\prime}}\right) V_{o}^{* *}(\vec{x}) U\left(\vec{x}^{\prime}, t\right) \\
L^{*}(\vec{x}, \vec{y}) L\left(\vec{x}^{\prime}, \vec{y}\right) d \vec{y} d \overrightarrow{x^{\prime}} d \vec{x}
\end{array}
$$

From Eq. ( 135$)$, the $\vec{y}$ integration gives rise to the delta function, $\delta\left(\overrightarrow{x^{\prime}}-\vec{x}\right)$. Then, performing the $\overrightarrow{x^{\prime}}$ integration, one obtains

$$
I_{Z}=\operatorname{Re} e^{j \omega t} \eta \int W(\vec{x}) V_{o}^{*}(\vec{x}) U(\vec{x}, t) d \vec{x}
$$

which is identical to the beat signal current $I_{U}$ as given by Eq. (151). Thus, not only is the maximum average signal-to-noise ratio the same, independent of the choice of the optical system, but so too is the time behavior of the corresponding beat signals.

The equivalence of these different heterodyning schemes can be seen from a very simple argument[17]. The crux of the argument lies in the fact that the beat signal current is unchanged by passing both the signal and local oscillator beams through, for example, a lens before combining them on the photodetector surface[1,23]. This equivalence is, of course, implied by the fact that $I_{Z}$, Eq. (152), is equal to $I_{U}$, Eq. (15l), and that $L(\vec{x}, \vec{y})$ represents a linear transformation. A rather simple argument then shows that: the optimum local oscillator beam for heterodyning with the received signal after it alone is passed through the lens, must be identical to the beam obtained by passing through the lens the optimum local oscillator beam for heterodyning with the received signal itself. It follows that no 
additional improvement in the average signal-to-noise ratio, indeed in the time behaviox of the beat signal itself, can be obtained by heterodyning with the received signal as it appears, for example, in the focal plane of a lens.

The above is not meant to imply that the optimum local oscillator beam discussed here, may not lead to a smallex signal power variance than, for example, that associated with the plane wave local oscillator beam assumed by Fried[ 1$]$ in his determination of average signal-tonoise ratio. Indeed, there is reas on to believe that the optimum local oscillator beam, tapered in amplitude as it is, may lead to a smaller signal power variance than that associated with the plane wave local oscillator as determined by Fried[21]. This point is presently under consideration. It can be noted here that one possible method for determining the signal power variance associated with the optimum beam is to note that, from Eqs. (5), (61), and (65), the beat signal current is, since $\left\{\Phi_{n}\right\}$ are orthonormal,

$$
(156) \quad I(t)=\operatorname{Re}\left(e^{j \omega t} \eta V_{q} A_{q}(t)\right)
$$

It follows then, that the beat signal power is, from Eq. (156)

$$
P(t)=\frac{1}{2} \eta^{2}\left|v_{q}\right|^{2}\left|A_{q}(t)\right|^{2}
$$

and hence that the, normalized, signal power variance $S$, defined by 


$$
S=\frac{\left\langle P^{2}(t)\right\rangle-\langle P(t)\rangle^{2}}{<P(t)\rangle^{2}}
$$

is, from Eq. (157)

$$
S=\frac{\left\langle\left|A_{q}(t)\right|^{4}\right\rangle-\left\langle\left|A_{q}(t)\right|^{2}\right\rangle^{2}}{\left.<\left|A_{q}(t)\right|^{2}\right\rangle^{2}}
$$

or

$$
S=\frac{\left\langle\left|A_{q}(t)\right|^{4}\right\rangle-\lambda_{\max }}{\lambda_{\max }}
$$

where $\lambda_{\max }=\left\langle\left|A_{q}(t)\right|^{2}\right\rangle$, is the maximum eigenvalue of the integral equation, Eq. (27). Once the form of the probability distribution function for $A_{q}(t)$ is known, then $S$ can easily be determined from Eq. $(160)$

It must be noted here that the "optimum" local oscillator beam determined above is not, necessarily, truly optimum in the sense that it affords both a "large" average signal-to-noise ratio and a "small" signal power variance. The truly optimum local oscillator beam might sacrafice maximum average signal-to-noise ratio to obtain a "happy medium" between average signal-to-noise ratio and signal power variance. This "happy medium" could, for example, be precisely defined as follows: Given a specific optical communications system, whose job is, for example, to transmit one of $M$ discrete messages, then determine that time invariant heterodyne detection 
scheme which minimizes the probability that the receiver makes an error in deciding which message was sent. Since, as described previously, the probability of error (or mean square error in the case of waveform communication) is a nonlinear function of the signal-to-noise ratio[ 8], the solution of this problem demands a knowledge of the probability distribution function of the beat signal power.

This Chapter can be summarized by stating that the "optimum" time invariant detection scheme can be realized by properly shaping the local oscillator beam alone. Thus, no additional improvement can be obtained by pre-processing the received optical signal. This applies not only to the average signal-to-noise ratio, but also to the variation of the signal-to-noise ratio about its average value.

In the next Chapter, recommendations for further study are given. 


\section{CHAPTER VII \\ COMMENTS AND RECOMMENDATIONS}

This Chapter presents recommendations for further extensions of this work. At this point let us review some of the problems of optical heterodyne detection. This review will lead to suggested projects.

A most serious difficulty encountered in heterodyning with an atmospherically distorted optical beam, even in the absence of amplitude fluctuations, is that the phase difference between any two "points" on the received signal may assume, in the course of time, many different values; the number of which increases from zero as the points are separated. This consideration gives some insight to the result obtained previously, viz., that no single, fixed local oscillator wavefront can cause the beat signals generated at these points to add in phase "most" of the time. That is, since the difference in phase between these individual beat signals is a linear function of the phase difference between the received signals at the corresponding points (a difference which may fluctuate through many radians for points not "too close" together), it is perhaps unreasonable to expect that these individual 
beat signals will add in phase and thus, produce a large detector output current "most" of the time. Even if this were possible, the amplitude fluctuations of the received signal would, even when the total received optical power remained constant, induce fluctuations in the output beat signal current. This effect too, can obviously not be entirely overcome by shaping the local oscillator beam.

In view of the difficulty, i. e., the random changes in the phase and amplitude of the received signal, it would appea $r$ that the following represent two most promising methods for obtaining improvement:

(1) Adjust, with time, the shape of the local oscillator amplitude and/or wavefront so that it matches the corresponding quantities of the received signal for all, or a large fraction, of time, or

(2) Use an array of "small", isolated detectors and adjust, with time, the amplitudes and/or phases of the beat signals generated by each one so that they add as would the individual beat signal currents generated at each "point" of the detector as in method one above.

These two methods are equivalent in the sense that each hits directly at the heart of the problem, namely, that of compensating, with time, for the phase and amplitude fluctuation of the received signal. They differ in that the first method accomplishes this before, and the second after, the individual beat signals are generated. 
There are, undoubtedly, many possible ways of accomplishing each of the above. For example, suppose that the atmospheric distortion of the received signal consists of a random tilting of an otherwise "nearly" flat wavefront. The local oscillator beam could, in theory, be made to follow this tilting by reflecting it from a flat servo controlled mirror whose orientation is adjusted until the beat signal reaches its maximum value. Alternatively, the heterodyning could be done in the focal plane of a lens through which the received signal is allowed to pass. By using a fixed local oscillator beam and activating only that portion of the phototube where the focused signal spot lies (as determined, for example, by focusing a small fraction of the received signal to an array of small detectors) then any tilting of the signal is tracked.

A somewhat more elaborate scheme, utilizing features of both methods one and two above, could be employed in a case where the atmospheric distortion can be considered as a random tilting over only each area of an array of adjacent areas of the received signal. In this case, the signal from each of these areas could be passed through a lens and focused to the surface of a photodetector, one detector for each area. Tilting is tracked by each of these detectors as discussed above. Finally, the amplitudes and/or phases of the beat signals from each detector could be automatically adjusted as described in method two above. 
As each of the above and other approaches will have their own advantages and difficulties, an evaluation of the performance of several of these offers an excellent area for future study.

A further investigation that should be undertaken is an experimental determination of the heterodyne signal level probability distribution function (for a time invariant local oscillator wave function). This data is, as explained in the Introduction, necessary for evaluating the performance of the time invariant detection system, and will also be useful for designing and evaluating time varying detection methods.

Furthermore, some thought should be devoted to the possibility of being able to use the results of heterodyne studies to evaluate the performance capabilities of other optical communication systems. As already noted, the Karhunen-Loeve theorem represents a powerful tool for such studies. Additional background material for this type of study can be found in Reference 22 . 


\section{CHAPTER VIII}

SUMMARY

The problem under consideration in this investigation was to determine that time invariant detection scheme which maximizes the average signal-to-noise ratio in the heterodyne detection of a randomly fluctuating optical signal beam. This problem has been solved. Indeed, this scheme can be realized by properly shaping both the isophase surface and the intensity pattern of the local oscillator beam. No signal pre-processing is required. Furthermore, it was shown that both the phase front and the amplitude shape of this "optimum" beam depend on only the two point or mutual coherence function of the received optical signal. This result, and its physical interpretations, which were derived formally by the calculus of variations, have also been shown to be simple consequences of the Karhunen-Loeve theorem.

Several examples were considered. The first showed that for the special case of a non-fluctuating received signal phase front and amplitude shape, the local oscillator beam should "match" the signal beam in both phase and amplitude shape for maximum average 
signal-to-noise ratio. Secondly, the general conditions under which the phase front of the optimum local oscillator beam is parallel to the average received signal phase front were stated. Furthermore, the basic phenomenon which leads to an aperture size above which the average signal-to-noise ratio does not increase significantly was indic ated.

For the case of an atmospherically distorted plane wave optical signal it was shown that the optimum local oscillator beam differs significantly from a plane wave. Indeed, it is a beam with a flat phase front, parallel to the average signal phase front, and an amplitude which is a maximum at the center of the receiver aperture and decreases to a minimum at the outer boundaries of this aperture. In particular, for the case of an aperture with diameter $r_{0}$ ( $r_{0}$ being Fried's[1] "optimum aperture diameter"), the ratio of maximum to minimum intensity is more than 6 to 1 .

However, even though this optimum beam differs markedly from a plane wave, it was shown that it does not significantly increase the average signal-to-noise ratio over that for the plane wave local oscillator beam as reported by Fried[ 1$]$. 
It should be emphasized here that it has been rigorously shown that no pre-processing of the distorted signal(which employs an optical system of the form discussed) can lead to a larger average signal-to-noise ratio, indeed to instantaneous values of the beat signal current, which could not be obtained without any such pre-processing. In Chapter VII of this investigation, several suggestions for future investigations were considered. 


\section{APPENDIX I \\ MAXIMUM $\left(\frac{S}{N}\right)$ - SCHWARZ INEQUA LITY}

The result given in Eq. (35). (namely, that the average beat signal power is a maximum when both beams have the same amplitude shape, as well as the same phase shape) is merely a statement of the Schwarz inequality. That is, with both $A(\vec{x})$ and $\phi(\vec{x})$ independent of time it follows from Eq. (7) that

$$
\begin{aligned}
P_{0} & =\frac{1}{2} n^{2} \iint W(\vec{x}) W\left(\overrightarrow{x^{\prime}}\right) V^{*}(\vec{x}) V\left(\overrightarrow{x^{\prime}}\right) U(\vec{x}) U^{*}\left(\overrightarrow{x^{\prime}}\right) d \vec{x} d \overrightarrow{x^{\prime}} \\
& =\frac{1}{2} \eta^{2}\left|\int W(\vec{x}) V^{*}(\vec{x}) U(\vec{x}) d \vec{x}\right|^{2},
\end{aligned}
$$

and maximization of $P_{o}$ subject to

$$
P_{L}=\frac{1}{2} \eta \int W(\vec{x}) V(\vec{x}) V^{*}(\vec{x}) d \vec{x}=\text { constant }
$$

is equivalent to maximization of the ratio

$$
\frac{P_{0}}{P_{L}}=\eta \frac{\left|\int W(\vec{x}) V^{*}(\vec{x}) U(\vec{x}) d \vec{x}\right|^{2}}{\int W(\vec{x}) V(\vec{x}) V^{*}(\vec{x}) d \vec{x}}
$$


Applying the Schwarz inequality to the numerator of Eq. (163) i i. e. ,

$$
\begin{gathered}
\left|\int W(\vec{x}) V^{*}(\vec{x}) U(\vec{x}) d \vec{x}\right|^{2} \leq \int W(\vec{x}) V(\vec{x}) V^{*}(\vec{x}) d \vec{x} \\
\cdot \int W(\vec{x}) U(\vec{x}) U^{*}(\vec{x}) d \vec{x},
\end{gathered}
$$

if follows that

$$
\frac{P_{0}}{P_{L}} \leq \eta \int W(\vec{x}) U(\vec{x}) U^{*}(\vec{x}) d \vec{x}
$$

The maximum value of $P_{O} / P_{L}$ then occurs when the equality sign holds and, as is well known, this condition obtains when the two functions appearing in the Schwarz inequality are proportional; i. e., when

$$
V(\vec{x})=C U(\vec{x})
$$

where $C$ is an arbitrary complex complex constant. Writing $C=|C| e^{j \theta}$ it follows from Eq. (166) that

$$
\theta(\vec{x})=\phi(\vec{x})+\theta_{0}
$$

and

$$
B(\vec{x})=|C| A(\vec{x})
$$

which are precisely the results obtained previously in this investigation. 


\section{APPENDIX II \\ REALIZATION OF OPTIMUM LOCAL \\ OSCILIATOR WAVE FUNCTION}

In this Appendix a brief discussion of one possible method for realizing the optimum fixed local oscillator wave function is given. In addition, an experimental arrangement for measuring the mutual coherence function is shown.

As shown in Fig. 11, the optimum local oscillator beam could be realized by passing a plane wave through an amplitude and phase filter whose transmission characteristics would be determined from the solution of the amplitude integral equation, Eq. (27), and the phase equation, Eq. (26). As mentioned above, the solution of each of these equations requires a knowledge of only the real and imaginary parts of the mutual coherence function; that is,

$$
\bar{C}\left(\vec{x}, \overrightarrow{x^{\prime}}\right)=\left\langle A(\vec{x}, t) A\left(\overrightarrow{x^{\prime}}, t\right) \cos \left(\phi(\vec{x}, t)-\phi\left(\overrightarrow{x^{\prime}}, t\right)\right)\right\rangle
$$

and

$$
\bar{S}\left(\vec{x}, \overrightarrow{x^{\prime}}\right)=\left\langle A(\vec{x}, t) A\left(\vec{x}^{\prime}, t\right) \sin \left(\phi(\vec{x}, t)-\phi\left(\overrightarrow{x^{\prime}}, t\right)\right)\right\rangle .
$$




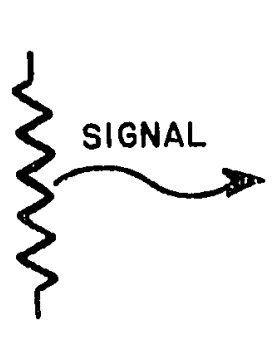

\section{BEAM SPLITTER}

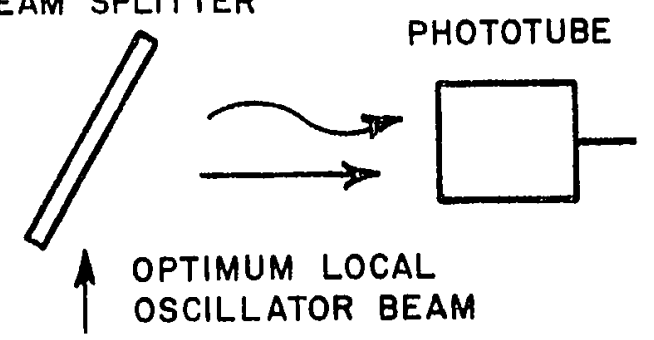

EIIIIIIX PHASE FILTER

SIIUIIUIJ AMPLITUDE FILTER

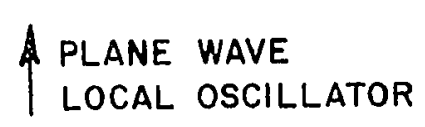

Fig. 11--Realization of optimum local oscillator beam.

Experimentally, these two functions could be determined by the scheme illustrated in Fig. 12. Here $V_{1}$ is the voltage generated by passing the output current of phototube one $\left(P_{1}\right)$ through a resistance of $\mathrm{C}$ ohms $/ \mathrm{m}^{2} . \Delta \mathrm{S}$ is the area of the phototube illuminated by the signal. and local oscillator fields passing through the small hole, located at position $\overrightarrow{x_{1}}$, in the opaque screen and $\eta[] \Delta S$ is the output current of $P_{1}$ generated by these fields. The remainder of Fig. 12 should be self-explanatory. By varying the location of the holes in the opaque screen, $\bar{C}$ and $\bar{S}$ can be obtained for all pairs of points on the received beam. The function of the computer is to determine the solution of the amplitude and phase equations; i. e., Eqs. (26) and (27), from a knowledge of its inputs, $\bar{C}$ and $\bar{S}$. 


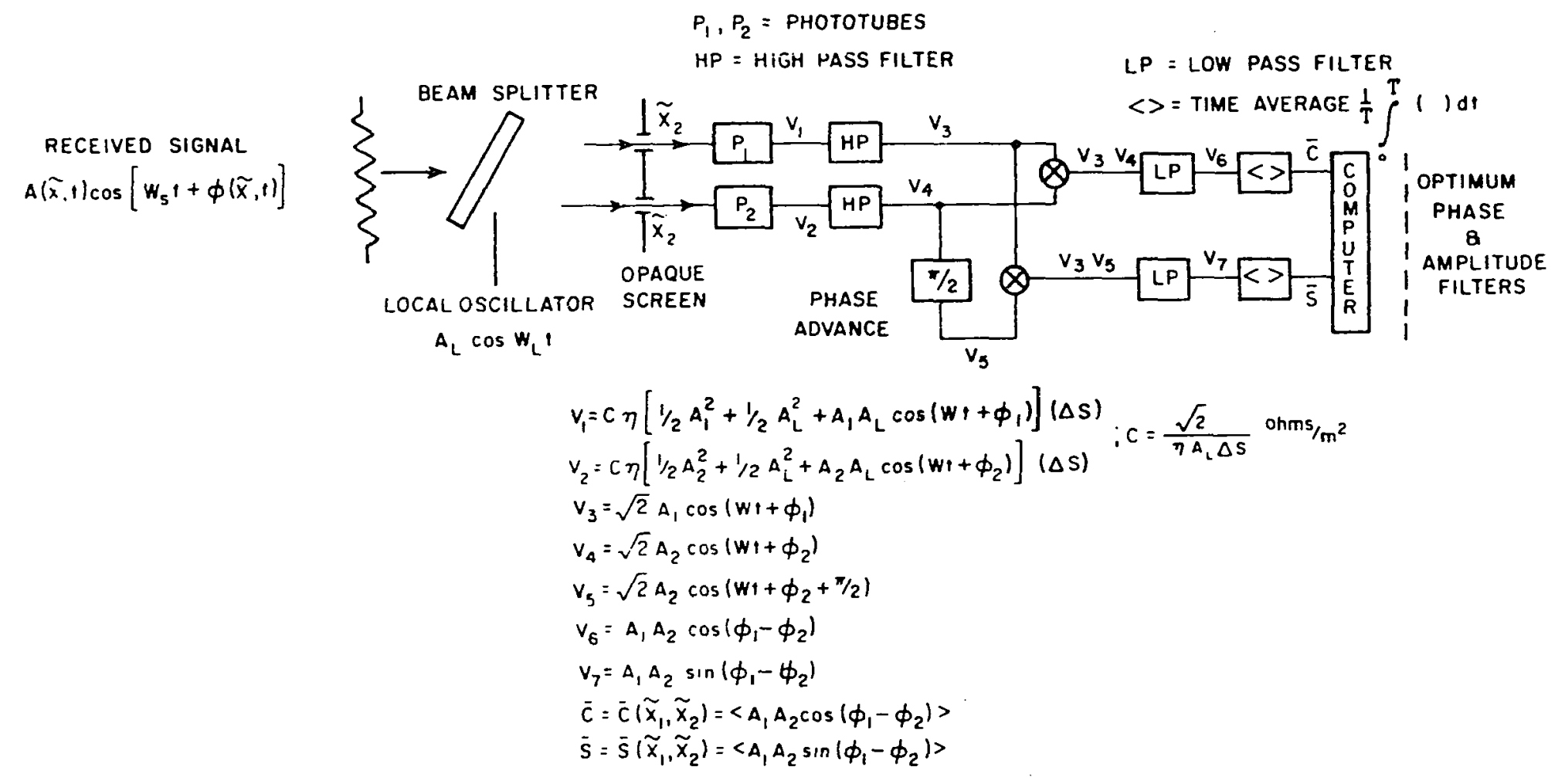

Fig. 12--Measurement of mutual coherence function. 


\section{APPENDIX III \\ MUTUAL COHERENCE FUNCTION}

This Appendix summarizes one of several different, theoretical derivations of the mutual coherence function of an atmospherically distorted optical plane wave. The derivation follows that suggested by Hufnagel and Stanley's paper[15] together with the needed modifications discussed by Chase[ 18] and Fried[ 19].

The physical situation under consideration is the following: A plane monochromatic optical wave, $R e U_{0} e^{j \omega_{s} t}$, is incident on a region of randomly fluctuating index of refraction. As a result of these fluctuations, there appear[14] irregular changes in the phase and hence, amplitude of the optical field, $\operatorname{Re} U(\vec{r}, t) e^{j \omega_{s} t}$. The quantity of interest is the mutual coherence function, $M_{U}$, defined as

$$
M_{U}\left(\overrightarrow{\rho_{1}}, \overrightarrow{\rho_{2}}, Z\right)=\left\langle U\left(\overrightarrow{\rho_{1}}, Z, t\right) U^{*}\left(\overrightarrow{\rho_{2}}, Z, t\right)\right\rangle
$$

where $\vec{\rho}_{i}$ denotes a position in a plane of constant $Z$ ( $Z$ being the direction of propagation of the unperturbed wave) and the angle brackets denote a time average over an interval of length $\mathrm{T}$. 
To obtain an exact solution of this problem requires solving the time dependent wave equation. The problem simplifies, however, by assuming that the time changes in the index of refraction are sufficiently slow (compared with the optical frequency) so that the frequency fluctuations of the optical field may be ignored[21]. With this assumption the general procedure has been to model the physical situation by an emsemble of systems each with a spatially varying, but time independent index of refraction. In other words, the index of refraction within the media is considered to be a time independent random function of position. The time changes in the index of refraction which actually occur are then regarded as changes in the different realizations of the random index of refraction field, $n(\vec{r}, \beta)$. $\beta$ is a parameter used to distinguish between different members of the ensemble. It is assumed that the time average in Eq. (168) may be replaced by an ensemble average so that

$$
M_{u}\left(\vec{\rho}_{1}, \vec{\rho}_{2}, Z\right)=\left\langle U\left(\overrightarrow{\rho_{1}}, Z, \beta\right) U^{*}\left(\overrightarrow{\rho_{2}}, Z, \beta\right)\right\rangle
$$

where $\operatorname{Re} U(\vec{p}, Z, \beta) e^{j \omega_{s} t}$ is the optical field associated with the index of refraction ensemble member $n(\vec{r}, \beta)$, and the brackets now denote an ensemble average. What this model neglects is the "transit" behavior of the fields which occurs, in the physical system, when the 
index of refraction function changes from, say $n\left(\vec{r}, \beta_{1}\right)$ to $n\left(\vec{r}, \beta_{2}\right)$. It will further be as sumed that changes in the polarization of the field are negligible[ 14].

With these as sumptions, the optical field at position $\vec{r}$, $\operatorname{Re} U(\vec{r}, \beta) e^{j \omega_{s} t}$, may be written in the form

$$
\operatorname{Re} U(\vec{x}, \beta) e^{j \omega_{s} t}=\operatorname{Re} A(\vec{x}, \beta) U_{0} e^{j \omega_{s} t}
$$

where $U_{O} e^{j \omega_{S} t}$ represents the unperturbed wave, with $U_{0}=A_{O} e^{-j k z}$, and $A(\vec{r}, \beta)$ describes the phase and amplitude perturbations of the wave associated with the refractive index function $n(\vec{r}, \beta)$. Assuming that the optical wavelength, $\lambda$, is "much" smaller than the geometrical dimension, $\ell_{0}$, of the smallest inhomogeneity in the spatial distribution of the refractive index, it follows that [14] U satisfies the (scalar) wave equation

$$
\nabla^{2} U(\vec{r}, \beta)+n^{2}(\vec{r}, \beta) k^{2} U(\vec{r}, \beta)=0 .
$$

Letting

$$
\mathrm{n}(\overrightarrow{\mathrm{r}}, \beta)=1+\mathrm{n}_{1}(\overrightarrow{\mathrm{r}}, \beta)
$$

and assuming that $\mathrm{n}_{1}<<1$, it follows, neglecting terms involving $n_{1}^{2}$, that $A(\vec{r}, \beta)$ satisfies the equation 


$$
\nabla^{2} A(\vec{r}, \beta)+2 i k \frac{\partial}{\partial Z} A(\vec{r}, \beta)+2 k^{2} n_{1}(\vec{r}, \beta) A(\vec{r}, \beta)=0
$$

In order to obtain an explicit relation between $M_{u}$ and $n_{1}(\vec{r}, \beta)$, Eq. $(174)$ could be solved for $A\left(\vec{p}_{1}, Z, \beta\right)$ and $A^{* *}\left(\vec{p}_{2}, Z, \beta\right)$ and their product then averaged to for $\mathrm{m} \mathrm{M}_{\mathrm{u}}$. Several methods for obtaining an approximate solution in this manner appear in Reference 15. Hufnagel and Stanley[15], however, suggested that one try first to obtain a differential equation involving the product, $A\left(\vec{\rho}_{1}, Z, \beta\right) A *\left(\vec{\rho}_{2}, Z, \beta\right)$. The resulting equation is then to be averaged term by term and then, hopefully, directly solvable for $M_{U}$. This equation for $A\left(\vec{\rho}_{1}, Z, R\right)$ $A^{*}\left(\overrightarrow{\rho_{2}}, Z, \beta\right)$ can be obtained from Eq. (174). From that equation it follows that $A\left(\vec{\rho}_{1}, Z, P\right)$ satisfies the equation

$$
\begin{aligned}
\nabla^{2} A\left(\vec{p}_{1}, Z, \beta\right)+2 i k & \frac{\partial}{\partial Z} A\left(\vec{p}_{1}, Z, \beta\right) \\
+ & 2 k^{2} n_{1}\left(\vec{p}_{1}, Z, \beta\right) A\left(\vec{p}_{1}, Z, \beta\right)=0,
\end{aligned}
$$

where $\left.\nabla^{2} A\left(\overrightarrow{\rho_{1}}, Z, \beta\right) \equiv \nabla^{2} A(\vec{r}, \beta)\right|_{r}=\left(\overrightarrow{\rho_{1}}, Z\right)$, etc. , while $A^{*}\left(\overrightarrow{\rho_{2}}, Z, \beta\right)$ satisfies the equation

$$
\begin{aligned}
\nabla^{2} A^{*}\left(\vec{p}_{2}, Z, \beta\right)- & 2 i k \frac{\partial}{\partial Z} A^{*}\left(\vec{p}_{2}, Z, \beta\right) \\
& +2 k^{2} n_{1}\left(\vec{p}_{2}, Z, \beta\right) A^{*}\left(\vec{p}_{2}, Z, \beta\right)=0 .
\end{aligned}
$$


Multiplying each term of Eq. $(175)$ by $A^{*}\left(\vec{p}_{2}, Z, \beta\right)$ and of Eq. (176) by $A\left(\overrightarrow{\rho_{1}}, Z, \beta\right)$ it follows, upon subtracting the resulting equations, that

$$
\begin{aligned}
& A^{*}\left(\overrightarrow{\rho_{2}}, Z, \beta\right) \nabla^{2} A\left(\overrightarrow{\rho_{1}}, Z, \beta\right)-A\left(\overrightarrow{\rho_{1}}, Z, \beta\right) \nabla^{2} A^{*}\left(\overrightarrow{\rho_{2}}, Z, \beta\right) \\
& +2 i k \frac{\partial}{\partial Z}\left[A\left(\overrightarrow{\rho_{1}}, Z, \rho\right) A^{*}\left(\overrightarrow{\rho_{2}}, Z, \beta\right)\right] \\
& +2 k^{2}\left[n_{1}\left(\overrightarrow{\rho_{1}}, Z, \rho\right)-n_{1}\left(\overrightarrow{\rho_{2}}, Z, \beta\right)\right] A\left(\vec{p}_{1}, Z, \beta\right) A^{*}\left(\overrightarrow{p_{2}}, Z, \beta\right)=0 .
\end{aligned}
$$

Equation (177) almost has the desired form; all but the first two terms invoive only the product of $A\left(\vec{p}_{1}, Z, \beta\right)$ and $A *\left(\overrightarrow{p_{2}}, Z, \beta\right)$. However, upon averaging Eq. (177) term by term and making use of the as sumption that

$$
\begin{aligned}
& <A^{*}\left(\overrightarrow{\rho_{2}}, Z, f\right) \nabla^{2} A\left(\overrightarrow{\rho_{1}}, Z, \beta\right)> \\
& =\left\langle A\left(\overrightarrow{\rho_{1}}, Z, f\right) \nabla^{2} A^{*}\left(\overrightarrow{\rho_{2}}, Z, f\right)>,\right.
\end{aligned}
$$

Hufnagel and Stanley obtained

$$
\begin{aligned}
\frac{\partial}{\partial Z}<A & \left(\overrightarrow{\rho_{1}}, Z, \beta\right) A^{*}\left(\overrightarrow{\rho_{2}}, Z, \beta\right)> \\
-i k< & {\left[n_{1}\left(\overrightarrow{\rho_{1}}, Z, \beta\right)-n_{1}\left(\vec{\rho}_{2}, Z, \beta\right)\right] } \\
& {\left[A\left(\overrightarrow{\rho_{1}}, Z, \beta\right) A^{*}\left(\overrightarrow{\rho_{2}}, Z, f\right)\right]>=0 . }
\end{aligned}
$$


Hufnagel and Stanley claimed that a solution of Eq. (179) for $<A\left(\vec{p}_{1}, Z, \beta\right) A^{*}\left(\overrightarrow{p_{2}}, Z, \beta\right)>$, or, assuming $\left|U_{0}\right|=1$, for $M_{u}$ is

$$
\begin{aligned}
& M_{u}\left(\vec{p}_{1}, \vec{p}_{2}, Z\right)=\left\langle A\left(\vec{p}_{1}, Z, \beta\right) A^{*}\left(\vec{p}_{2}, Z, \beta\right)\right\rangle \\
& =\left\langle\exp \left\{i k \int_{0}^{Z}\left[n_{1}\left(\vec{p}_{1}, Z^{\prime}, \beta\right)-n_{1}\left(\vec{p}_{2}, Z^{\prime}, \beta\right)\right] d Z^{\prime}\right\}\right\rangle
\end{aligned}
$$

Substituting this assumed solution for $M_{\mathrm{u}}$ into those terms in which it appears in Eq. (179) gives

$$
\begin{aligned}
& <\left[n_{1}\left(\overrightarrow{\rho_{1}}, Z, \beta\right)-n_{1}\left(\overrightarrow{\rho_{2}}, Z, \beta\right)\right] \\
& \left.\left[\exp \left\{i k \int_{0}^{Z}\left[n_{1}\left(\overrightarrow{\rho_{1}}, Z^{\prime}, \beta\right)-n_{1}\left(\overrightarrow{\rho_{2}}, Z^{\prime}, \beta\right)\right] d Z\right\}\right]\right\rangle \\
& \stackrel{?}{=}\left\langle\left[n_{1}\left(\overrightarrow{\rho_{1}}, Z, \beta\right)-n_{1}\left(\overrightarrow{\rho_{2}}, Z, \beta\right)\right]\left[A\left(\overrightarrow{\rho_{1}}, Z, \beta\right) A^{* ; k}\left(\overrightarrow{\rho_{2}}, Z, \beta\right)\right]\right\rangle .
\end{aligned}
$$

Note that $A\left(\overrightarrow{\rho_{1}}, Z, f\right) A^{* *}\left(\overrightarrow{\rho_{2}}, Z, \beta\right)$ appearing in the second term of Eq. (180) cannot, in general, be replaced by

$$
\exp \left\{i k \int_{0}^{Z}\left[n_{1}\left(\overrightarrow{\rho_{1}}, Z^{\prime}, \beta\right)-n_{1}\left(\overrightarrow{\rho_{2}}, Z^{\prime}, \beta\right)\right] d Z^{\prime}\right\}
$$

since, as noted by Hufnagel and Stanley, Eq. (180) does not, in general, imply equality of the quantities within the averaging brackets. Indeed, these quantities are equal only when the amplitude fluctuations of the optical field can be neglected. That is, if the path length ( $Z$ ) is sufficiently short so that geometrical optics may be applied to obtain 
the optical field for each realization of the random field $A(\vec{r}, \beta)$ then it follows that

$$
A\left(\vec{\rho}_{1}, Z, \beta\right)=\exp \left\{i \triangle \phi\left(\vec{\rho}_{1}, Z\right)\right\}
$$

where

$$
\Delta \phi\left(\overrightarrow{\rho_{1}}, Z\right)=k \int_{0}^{Z} n_{1}\left(\overrightarrow{p_{1}}, Z^{\prime}, \beta\right) d Z^{\prime}
$$

is the difference in phase between the perturbed and the unperturbed wave (the latter being that which would be present in the absence of turbulencel.

It should be noted here that the form given for $M_{u}$ by Hufnagel and Stanley is, except for some disagreement on its range of validity, generally agreed to be approximately correct. This is claimed to be the case even when geometrical optics cannot be applied to determine the fields for individual realizations of the random field $n(r, \beta)$. The question of interest here is to determine if this result can be deduced by the method suggested by Hufnagel and Stanley.

Chase[18], in his comments on Hufnagel and Stanley's paper suggested that Eq. (177) be solved as a first order linear differential equation for $A\left(\overrightarrow{p_{1}}, Z, \beta\right) A^{*}\left(\overrightarrow{\rho_{2}}, Z, \beta\right)$ and that the resulting solution then be averaged to form $M_{u}$. Thus, adopting Chase's notation let 


$$
f(Z, \beta)=A\left(\vec{\rho}_{1}, Z, \beta\right) A^{*}\left(\vec{p}_{2}, Z, \beta\right)
$$

and

$$
\begin{aligned}
h(Z, \beta)= & -(i 2 k)^{-1}\left[A^{*}\left(\overrightarrow{\rho_{2}}, Z, \beta\right) \nabla^{2} A\left(\overrightarrow{\rho_{1}}, Z, \beta\right)\right. \\
& \left.-A\left(\overrightarrow{\rho_{1}}, Z, \beta\right) \nabla^{2} A^{*}\left(\overrightarrow{\rho_{2}}, Z, \beta\right)\right]
\end{aligned}
$$

so that Eq. (177) takes the form

$$
\frac{\partial}{\partial Z} f(Z, \beta)+g(Z, \beta) f(Z, \beta)=h(Z, \beta)
$$

Chase gave as a solution for this equation

$$
\begin{aligned}
f(Z, \beta) & =\int_{0}^{Z} d Z^{\prime} h\left(Z^{\prime}, \beta\right) \exp \left[-\int_{Z^{\prime}}^{Z} d Z^{\prime \prime} g\left(Z^{\prime \prime}, \beta\right)\right] \\
& +f(0, \beta) \exp \left[-\int_{0}^{Z} d Z^{\prime \prime} g\left(Z^{\prime \prime}, \beta\right)\right]
\end{aligned}
$$

where $f(0, \beta)=1$ if the optical wave is unperturbed at $Z=0$.

Averaging Eq. ( 186$)$ term by term gives

(187)

$$
\begin{aligned}
M_{u}\left(\vec{\rho}_{1}, \vec{p}_{2}, Z\right) & =\langle f(Z, \beta)\rangle \\
= & \left.<\int_{0}^{Z} d Z^{\prime} h\left(Z^{\prime}, \beta\right) \exp \left[-\int_{Z^{\prime}}^{Z} d Z^{\prime \prime} g\left(Z^{\prime \prime}, \beta\right)\right]\right\rangle \\
+ & \left.<\exp \left[-\int_{0}^{Z} d Z^{\prime \prime} g\left(Z^{\prime \prime}, \beta\right)\right]\right\rangle
\end{aligned}
$$


Note that it is only the second term in Eq. (187) which is given by Eq. (180) for $\mathrm{M}_{\mathrm{u}}$. As noted by Chase, however, the assumption that $\langle\mathrm{h}(\mathrm{Z}, \beta)\rangle=0$, as given by Eq. $(178)$, does not imply the vanishing of the first term in Eq. (187). Chase argued this on the grounds that there is some, non zero, correlation between $h\left(Z^{\prime}, \beta\right)$ and $\int_{Z^{\prime}}^{Z} d Z^{\prime \prime} g\left(Z^{\prime \prime}, \beta\right)$. That such is true follows from the facts that:

(1) $h\left(Z^{\prime}, \beta\right)$ depends on the index of refraction between 0 and $Z^{\prime}$ while $\int^{2}$ $d Z^{\prime \prime} g\left(Z^{\prime}, \beta\right)$ depends on the index of refraction between $Z^{\prime}$ and $Z$, $\mathrm{Z}^{\prime}$

and (2) there is some non zero correlation between the index of refraction in the se two regions.

Fried[19], commenting on Chase's letter, argued, however, that since the range of correlation of the refractive index $\left(\ell_{0}\right)$ is very small compared to the total path length ( L) then the correlation between $h\left(Z^{\prime}, \beta\right)$ and $\int_{Z^{\prime}}^{Z} d Z^{\prime \prime} g\left(Z^{\prime \prime}, \beta\right)$ is (for most values of $Z^{\prime}$ ) very small. In particular, Fried[19], claims that the cross correlation product is of the order $l_{0}^{2}\left\langle n_{1}^{2}\right\rangle$ and hence, can be dropped due to the smallness of $\left\langle\mathrm{n}_{1}^{2}\right\rangle$. This type of argument, Fried[19] notes, is equivalent to Bourett's hypothesis of local independence, which amounts to regarding the "stochastic" field $n(\vec{r}, \beta)$ as having a negligible local effect on the 
perturbed field $U(\vec{r}, \beta)$. If Fried's argument applies, then the assumption that $\left\langle h\left(Z^{\prime}, \beta\right)\right\rangle=0$, as given by Eq. (177), is sufficient to establish the approximate accuracy of the solution for $M_{u}$ given by Hufnagel and Stanley.

Accepting that $M_{\mathrm{u}}$ is, approximately, given by Eq. (180), we proceed with the derivation of Hufnagel and Stanley. Thus, the quantity $S$ defined by

$$
S\left(\overrightarrow{p_{1}}, \overrightarrow{p_{2}}\right)=\int_{0}^{Z}\left[n_{1}\left(\overrightarrow{p_{1}}, Z^{\prime}, \beta\right)-n_{1}\left(\vec{p}_{2}, Z^{\prime}, \beta\right)\right] d Z^{\prime}
$$

is assumed to have a Gaussian distribution (by central limit theorem) with zero mean (i. e., $\left.\langle\mathrm{n}(\overrightarrow{\mathrm{r}}, \beta)\rangle=1 ; \therefore\left\langle\mathrm{n}_{1}(\overrightarrow{\mathrm{r}}, \beta)\right\rangle=0\right)$. It follows from this that

$$
<\exp i k S>=\exp \left(-\frac{1}{2} k^{2}<S^{2}>\right)
$$

where

$$
\begin{gathered}
\left\langle S^{2}\right\rangle=\int_{0}^{Z} d Z_{1} \int_{0}^{Z} d Z_{2}<\left[n_{1}\left(\vec{\rho}_{1}, Z_{1}, \beta\right)-n_{1}\left(\vec{p}_{2}, Z_{1}, \beta\right)\right] \\
\left.\quad\left[n_{1}\left(\overrightarrow{\rho_{1}}, z_{2}, \beta\right)-n_{1}\left(\overrightarrow{p_{2}}, z_{2}, \beta\right)\right]\right\rangle
\end{gathered}
$$

By making use of the identity $(a-b)(c-d)=\frac{1}{2}\left[(a-d)^{2}+(b-c)^{2}-(a-c)^{2}\right.$ $\left.-(b-d)^{2}\right]$ the integrand in Eq. (190) can be expressed in terms of the index of refraction structure function, $D_{n}$, defined as

$$
D_{n}=\left\langle\left[n_{1}\left(\overrightarrow{\rho_{i}}, z_{j}, f\right)-n_{1}\left(\overrightarrow{\rho_{k}}, z_{l}, \beta\right)\right]^{2}\right\rangle
$$


As a consequence of the assumptions of local isotropy and lateral stationariness together with the Kolmogoroff theory of turbulence, it follows that

$$
D_{n}=D_{n}(r)=C_{N}^{2} r^{2 / 3}
$$

where $C_{N}^{2}$ is called the refractive-index structure constant and $r$ is the distance between the points in question. Actually[ 15], Eq. (192) is valid only over the range $\ell_{0}<r<L_{0}$ where $\ell_{0}(\leq 3 \mathrm{~cm})$ and $L_{0}\left(\geq 10^{3} \mathrm{~cm}\right)$ are referred to as the inner and outer scales of turbulence. Neglecting this restriction, Hufnagel and Stanley show that the mutual coherence function is

$$
\begin{aligned}
M_{u}\left(\rho_{1}, \rho_{2}, Z\right) & =M_{u}(\rho, Z) \\
& =\exp \left[-\left(\frac{1}{2}\right)(2.91) k^{2} \rho^{5 / 3} \int_{0}^{Z} \mathrm{dZ} C_{n}^{2}\right] .
\end{aligned}
$$

Equation (125) has been independently obtained by Fried[1] . Comparing this with the form given by Fried[1], and used in this investigation, viz., from Eq. (97)

$$
M_{u}(\rho, Z)=\exp \left[-\frac{1}{2}(6.88)\left(\rho / r_{0}\right)^{5 / 3}\right]
$$

it follows that, for a path of length $R$,

$$
r_{0}=1.2 \times 10^{-3} \lambda_{\mathrm{u}}^{6 / 5} \mathrm{R}^{-3 / 5} \overline{\mathrm{C}}_{\mathrm{N}}^{-6 / 5} \quad \text { meters }
$$


where $\lambda_{u}$ is the wavelength in meters. In Fig. 13, the dependence of $r_{0}$ on $\lambda_{u}, R$, and $C_{N}^{2}$, as given by $\operatorname{Fried}[1]$, is shown graphically. This completes the derivation of the mutual coherence function for an atmospherically distorted optical plane wave. In closing it should be noted that, of those derivations which have come to the author's attention all seem to arrive at essentially the same form for the mutual coherence function. However, there is not a general agreement on its range of validity. In order to settle this issue the most reasonable approach would, perhaps, as noted by Fried[19] , be based on an experimental study. 


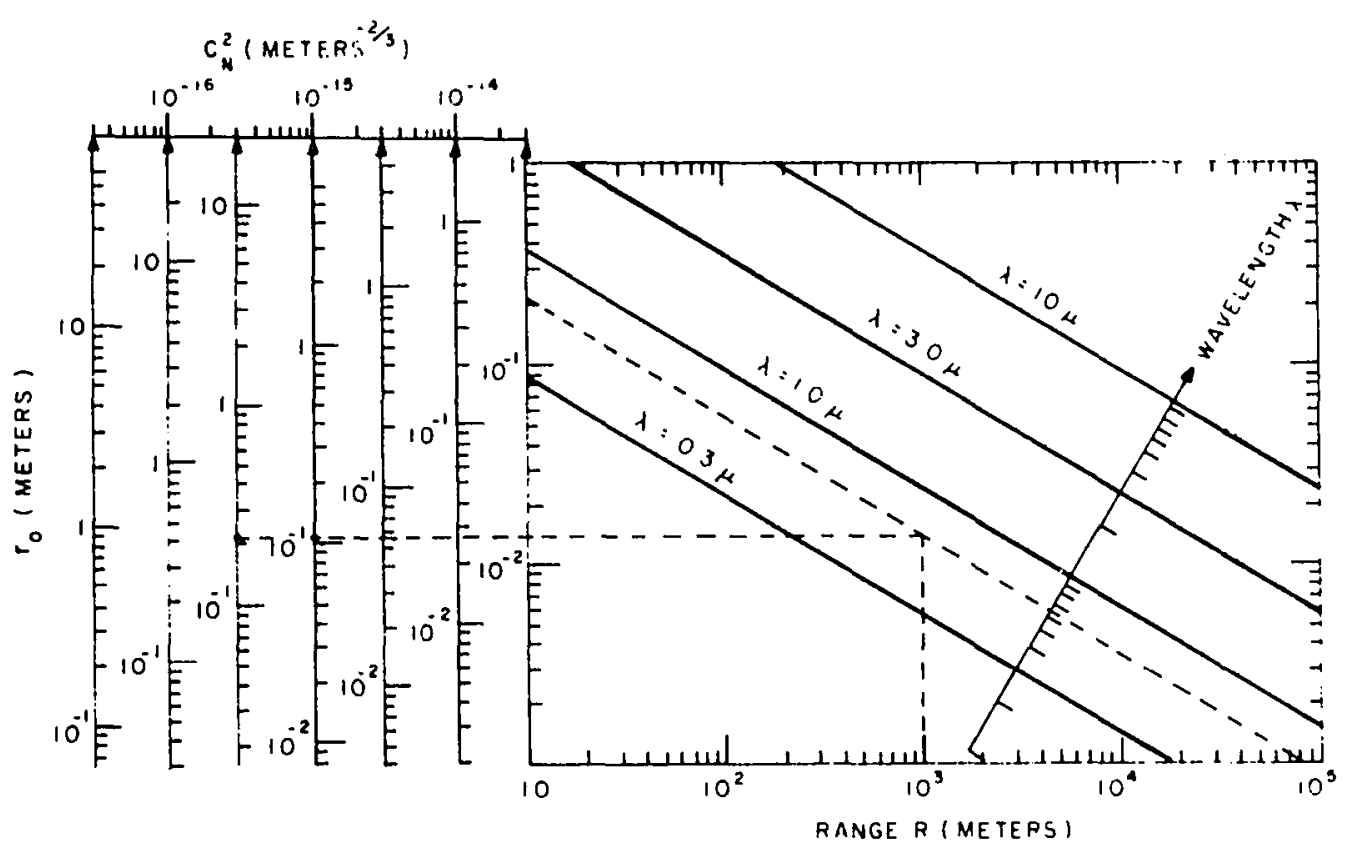

Fig. 13--Dependence of $r_{0}$ upon path length $R$, and strength of turbulence as measured by $C_{N}^{2}$ (from Reference 1 ).

* The $\mathrm{C}_{\mathrm{N}}^{2}$ scale in the upper left covers the range of values normally encountered within several tens of meters of the ground. To use the graph, a wavelength line is drawn parallel to the four heavy lines.and passing through the wavelength scale at the appropriate point. Where the vertical line, drawn through the range of interest, intercepts the wavelength line, we pass horizontally over to one of the vertical' $r_{0}$ scales on the left. Which of the $r_{0}$ scales we read from depends on the value of $C_{N}^{2}$ we wish to consider. As an example, we have drawn the wavelength line for $0.63 \mathrm{microns}$ land considered a range of one kilometer. For turbulence strength, denoted by $C_{N}^{2}=3 \times 10^{-16}$ to $\mathrm{C}_{\mathrm{N}}^{2}=10^{-15}$, we see that $\mathrm{r}_{\mathrm{o}}$ falls between approximately 0.21 and 0.11 meters. 
APPENDIX IV

\section{UNITARY TRANSFORMATIONS}

This Appendix discusses the unitary property of $L(\vec{x}, \vec{y})$ as given by Eq. (134).

The requirement that

$$
\begin{gathered}
\int d \vec{x} \int d \vec{x}^{\prime} V(\vec{x}, t) V^{*}\left(\overrightarrow{x^{\prime}}, t\right) I\left(\vec{x}, \vec{x}^{\prime}\right) \\
=\int V(\vec{x}, t) V^{*}(\vec{x}, t) d \vec{x}
\end{gathered}
$$

hold for all $V(\vec{x}, t)$ implies that

$$
I\left(\vec{x}, \overrightarrow{x^{\prime}}\right)=\delta\left(\vec{x}-\overrightarrow{x^{\prime}}\right)
$$

as stated in Eq. (135).

To show this, let $V(\vec{x}, t)=0$ except for $\vec{x}$ in the region $\Delta \overrightarrow{x_{i}}$ about $\vec{x}=\vec{x}_{i}$ and in the region $\Delta \overrightarrow{x_{j}}$ about $\vec{x}=\vec{x}_{j}$. Let $V(\vec{x}, t)$ equal $V_{i}$ in $\Delta \vec{x}_{i}$ and $v_{j}$ in $\Delta \vec{x}_{j}$. Then, Eq. (196) becomes, for small $\Delta \overrightarrow{\mathrm{x}}$,

$$
\begin{gathered}
V_{i} \Delta \vec{x}_{i} \int V\left(\overrightarrow{x^{\prime}}, t\right) I\left(\vec{x}_{i}, \vec{x}^{\prime}\right) d \vec{x}^{\prime}+V_{j} \Delta \vec{x}_{j} \int V\left(\overrightarrow{x^{\prime}}, t\right) I\left(\vec{x}_{j}, \vec{x}^{\prime}\right) \overrightarrow{d x^{\prime}} \\
=\left|V_{i}\right|^{2} \Delta \overrightarrow{x_{i}}+\left|V_{j}\right|^{2} \Delta \overrightarrow{x_{j}}
\end{gathered}
$$


or

(199)

$$
\begin{aligned}
V_{i} \Delta \vec{x}_{i} & \left\{V_{i}^{*} \Delta \overrightarrow{x_{i}} I\left(\vec{x}_{i}, \vec{x}_{j}\right)+V_{j}^{*} \Delta \vec{x}_{j} I\left(\vec{x}_{i}, \vec{x}_{j}\right)\right\} \\
& +V_{j} \Delta \overrightarrow{x_{j}}\left\{V_{i}^{*} \Delta \overrightarrow{x_{i}} I\left(\vec{x}_{j}, \vec{x}_{i}\right)+V_{j}^{*} \Delta \overrightarrow{x_{j}} I\left(\overrightarrow{x_{j}}, \overrightarrow{x_{i}}\right)\right\} \\
& =\left|V_{i}\right|^{2} \Delta \overrightarrow{x_{i}}+\left|V_{j}\right|^{2} \Delta \overrightarrow{x_{j}} .
\end{aligned}
$$

Since the above is to hold for all $V(\vec{x}, t)$ set $V_{j}=0$. Then Eq. (199) becomes

$$
\left|V_{i}\right|^{2}\left(\Delta \overrightarrow{x_{i}}\right)^{2} I\left(\overrightarrow{x_{i}}, \overrightarrow{x_{i}}\right)=\left|V_{i}\right|^{2} \Delta \overrightarrow{x_{i}}
$$

hence

$$
\text { (201) } I\left(\vec{x}_{i}, \vec{x}_{i}\right)=\frac{1}{\Delta \overrightarrow{x_{i}}}
$$

In view of Eq. (201), then Eq. (199) becomes

$$
V_{i} V_{j}^{*} I\left(\vec{x}_{i}, \vec{x}_{j}\right)+V_{j} V_{i}^{*} I\left(\vec{x}_{j}, \vec{x}_{i}\right)=0
$$

Again, since this is to hold for all $V(\vec{x}, t)$, let $V_{i}=1 ; V_{j}=i$ to obtain

$$
I\left(\vec{x}_{i}, \vec{x}_{j}\right)-I\left(\vec{x}_{j}, \vec{x}_{i}\right)=0
$$

Finally, letting $V_{i}=V_{j}=1$, Eq. (202) gives

$$
I\left(\vec{x}_{i}, \vec{x}_{j}\right)+I\left(\vec{x}_{j}, \vec{x}_{i}\right)=0
$$

From Eqs. (203) and (204) it follows that

$$
I\left(\vec{x}_{i}, \vec{x}_{j}\right)=0 ; \quad \vec{x}_{i} \neq \vec{x}_{j}
$$


Combining Eqs. (201) and (205) it follows that, for $\Delta \vec{x} \rightarrow 0, I(\vec{x}, \vec{x})$ has the property of the Dirac delta function, $\delta(\vec{x}-\vec{x})$, i. e. ,

(206) $\quad I\left(\vec{x}, \overrightarrow{x^{\prime}}\right)=0 ; \vec{x} \neq \vec{x}^{\prime}$,

and

(207) $\quad \int I\left(\vec{x}, \overrightarrow{x^{\prime}}\right) d \overrightarrow{x^{\prime}}=1$. 


\section{APPENDIX V \\ NORMALIZATION OF $\mathrm{S} / \mathrm{N}$}

This Appendix discusses the normalization of the average signalto-noise ratio as used in Chapter IV.

From Eqs. (5.12) and (5.13) of Fried[ 1], Fried's normalized signal-to-noise ratio, $\Psi\left(\mathrm{D} / \mathrm{r}_{0}\right)$, is, for a circular aperture of diameter $\left(\mathrm{D} / \mathrm{r}_{\mathrm{O}}\right)$,

$$
\Psi\left(D / r_{0}\right)=\frac{8}{\pi r_{0}^{2}(\eta / e) A^{2}}(S / N)
$$

In order to compare the $\mathrm{S} / \mathrm{N}$ computed in this investigation with that given by Fried, the normalized signal-to-noise ratio

$$
\gamma=\frac{8}{4 r_{0}^{2}(\eta / e) A^{2}}(S / N)
$$

is plotted. The factor of "4" replaces the factor of " $\pi$ " since a square aperture is being considered here.

Equation (209) may be written in the form

$$
\gamma=\beta^{2} \frac{2}{\eta / e \bar{A}^{2} \Delta S}(S / N)
$$

where

(211) $\quad \beta=\frac{\sqrt{\Delta S}}{r_{0}}$ 
From Eq. (18), the signal-to-noise ratio is given in terms of $\lambda_{\max }$ by

(212) $\quad(S / N)=\frac{\eta}{2 e} \lambda_{\max }$

and hence, Eq. (210) may be written

$(213)$

$$
\gamma=\beta^{2}\left(\frac{\lambda_{\max }}{\bar{A}^{2} \Delta S}\right)=\beta^{2} \lambda_{\max }^{\prime}
$$

as stated in Eq. (111). 


\section{REFERENCES}

1. Fried, D. L., "Optical Heterodyne Detection of an Atmospherically Distorted Signal Wave Front," Proc. IEEE-55, January 1967, pp. $57-67$.

2. Gardner, J., "Some Effects of Atmospheric Turbulence on Optical Heterodyne Communication," 1964 IEEE International Convention Record 12, pt. 6, pp. 337-342.

3. Kompfer, R. , "Optical Communications," Science, Vol. 150 , No. 3693, October 8, 1965, pp. 149-155.

4. Ross, Monte, Laser Receivers, Devices, Techniques, Systems, John Wiley and Sons, Inc., 1966.

5. See, for example, R. F. Lucy, "An Experimental Photomixer Image Tube," Proc. IEEE-51, January 1963, pp. 162-165.

6. See, for example, J. M. Wozencraft and I. M. Jacobs, Principles of Communication Engineering, John Wiley and Sons, Inc., New York, Chapter 4, 1965.

7. Ibid, Chapter 8 .

8. Ibid, Section 7. 3 . 
9. Chase, D. M. , "Power Loss in Propagation Through a Turbulent Medium for an Optical Heterodyne System with Angle Tracking," J. Opt. Soc. Am. , 56, January 1966, pp. 33-44.

10. Fried, D. L. , "Optical Resolution Through a Randomly Inhomogeneous Medium for Very Long and Very Short Exposures," J. Opt. Soc. Am. , 56, October 1966, pp. 1372-1379.

11. Fried, D. L., "Statistics of a Geometric Representation of Wavefront Distortion," J. Opt. Soc. Am. , 55, November 1965, pp. $1427-1438$.

12. Papoulis, Athanasios, Probability, Random Variables, and Stochastic Processes, McGraw-Hill Book Co. , Inc., New York, 1965, Chapter 13.

13. Davenport, W. B. ,Jr. and Root, W. L. , An Introduction to the Theory of Random Signals and Noise, McGraw-Hill Book Co. , Inc., New York, 1958, Chapter 6.

14. Tatarski, V.I., Wave Propagation in a Turbulent Medium, McGraw-Hill Book Co., Inc., New York, 1961, Section 12. 1.

15. Hufnagel, R. E. and Stanley, N. D. , "Modulation Transfer Function Associated with Image Transmission Through Turbulent Media," J. Opt. Soc. Am. , 54, January 1964, pp. 52-61.

16. Born, M. and Wolf, E., Principles of Optics, Pergamon Press, New York, 1959, Section 8.3 
17. This argument was first indicated to the author by D. L. Fried, private communication.

18. Chase, D. M. , "Coherence Function for Waves in Random Media," J. Opt. Soc. Am. , 55, November 1965, p. 1559.

19. Fried, D. L., "A Diffusion Analysis for the Propagation of Mutual Coherence," unpublished notes.

20. The suggestion to express this sum in the form $\vec{A} \cdot \vec{B}$ represents a valuable contribution of Frederick W. Gebhardt - private communication.

21. Fried, D. L., "Atmospheric Modulation Noise in an Optical Heterodyne Receiver," IEEE Journal of Quantum Electronics, June 1967, p. 213.

22. Gamo, H. , Progress in Optics, Volume 3, Edited by Wolf, E. North-Holland Publishing Company-Amsterdam, 1964, Chapter 3.

23. Siegman, A. E., "Antenna Properties of Optical Heterodyne Receivers," Proc. IEEE, Vol. 54, No. 10, October 1966, p. 1350 .

24. Loeve, M. , Probability Theory, D. Van Nostrand, Princeton, New Jersey, 1955.

25. Courant, R. and Hilbert, D. , Methods of Mathematical Physics, Interscience Publishers, New York, 1953. 